Geographical

breakdown of current account 


\section{Q 1 Current account}

Summary transactions in 2010

\begin{tabular}{|c|c|c|c|c|c|}
\hline & $\begin{array}{r}\text { Trade in } \\
\text { goods }\end{array}$ & $\begin{array}{l}\text { Trade in } \\
\text { services }\end{array}$ & Income & $\begin{array}{l}\text { Current } \\
\text { transfers }\end{array}$ & $\begin{array}{l}\text { Current } \\
\text { account }\end{array}$ \\
\hline \multicolumn{6}{|l|}{ Credits } \\
\hline \multicolumn{6}{|l|}{$\begin{array}{l}\text { Europe } \\
\text { European Union (EU) }\end{array}$} \\
\hline $\begin{array}{l}\text { Europeanan Union (EU) } \\
\text { Austria }\end{array}$ & 1463 & 643 & 401 & 37 & 2544 \\
\hline Belgium & 13377 & 2888 & 2440 & 217 & 18922 \\
\hline $\begin{array}{l}\text { Bulgaria } \\
\text { Cyprus }\end{array}$ & $\begin{array}{l}249 \\
562\end{array}$ & $\begin{array}{r}164 \\
602\end{array}$ & $\begin{array}{l}14 \\
70\end{array}$ & $\begin{array}{r}1 \\
9 \\
8\end{array}$ & $\begin{array}{r}436 \\
1242\end{array}$ \\
\hline Czech Republic & 1827 & 452 & -96 & 13 & $\begin{array}{l}1242 \\
2196\end{array}$ \\
\hline Denmark & 2757 & 2910 & 1124 & 46 & 6837 \\
\hline Estonia & 193 & 79 & 13 & 1 & 286 \\
\hline $\begin{array}{l}\text { Finland } \\
\text { France }\end{array}$ & 1498 & 1274 & 621 & 19 & $\begin{array}{r}3412 \\
34512\end{array}$ \\
\hline $\begin{array}{l}\text { France } \\
\text { Germany }\end{array}$ & $\begin{array}{l}19228 \\
27913\end{array}$ & 8913 & $\begin{array}{l}10078 \\
8235\end{array}$ & 324 & $\begin{array}{l}38543 \\
47388\end{array}$ \\
\hline $\begin{array}{l}\text { Germany } \\
\text { Greece }\end{array}$ & $\begin{array}{r}27913 \\
1381\end{array}$ & $\begin{array}{r}10800 \\
1119\end{array}$ & $\begin{array}{r}8235 \\
402\end{array}$ & $\begin{array}{r}440 \\
66\end{array}$ & $\begin{array}{r}47388 \\
2968\end{array}$ \\
\hline Hungary & 1082 & 472 & 102 & 9 & $\begin{array}{l}17605 \\
1667\end{array}$ \\
\hline $\begin{array}{l}\text { Ireland } \\
\text { Ittaly }\end{array}$ & 16932 & 8389 & -5426 & 182 & 20077 \\
\hline $\begin{array}{l}\text { Italy } \\
\text { Latvia }\end{array}$ & $\begin{array}{r}8853 \\
168\end{array}$ & $\begin{array}{r}5151 \\
87\end{array}$ & $\begin{array}{r}3693 \\
-56\end{array}$ & $\begin{aligned} 171 \\
7\end{aligned}$ & $\begin{array}{r}17868 \\
206\end{array}$ \\
\hline Lithuania & 225 & $\begin{array}{l}01 \\
96\end{array}$ & 14 & 20 & 355 \\
\hline Luxembourg & 246 & 2144 & 11749 & 25 & 14164 \\
\hline Malta & 393 & 227 & 65 & 10 & 695 \\
\hline $\begin{array}{l}\text { Netherlands } \\
\text { Poland }\end{array}$ & $\begin{array}{r}21250 \\
3804\end{array}$ & $\begin{array}{r}10069 \\
1132\end{array}$ & $\begin{array}{r}12025 \\
569\end{array}$ & $\begin{array}{r}363 \\
54\end{array}$ & $\begin{array}{r}43707 \\
5559\end{array}$ \\
\hline $\begin{array}{l}\text { Poland } \\
\text { Portugal }\end{array}$ & $\begin{array}{l}3804 \\
1837\end{array}$ & $\begin{array}{l}1732 \\
779\end{array}$ & $\begin{array}{l}569 \\
582\end{array}$ & $\begin{array}{l}54 \\
25\end{array}$ & $\begin{array}{l}5559 \\
3223\end{array}$ \\
\hline Romania & 781 & 359 & 114 & 6 & 1260 \\
\hline $\begin{array}{l}\text { Slovak Republic } \\
\text { Spvenia }\end{array}$ & 466 & 291 & 52 & 9 & 818 \\
\hline $\begin{array}{l}\text { Slovenia } \\
\text { Spain }\end{array}$ & $\begin{array}{r}220 \\
9940\end{array}$ & 92 & 3159 & ${ }^{2} 1$ & $\begin{array}{r}308 \\
18399\end{array}$ \\
\hline $\begin{array}{l}\text { Spain } \\
\text { Sweden }\end{array}$ & $\begin{array}{l}9940 \\
5563\end{array}$ & $\begin{array}{l}5139 \\
2911\end{array}$ & $\begin{array}{l}3159 \\
3220\end{array}$ & $\begin{array}{l}161 \\
91\end{array}$ & $\begin{array}{l}18399 \\
11785\end{array}$ \\
\hline European Central Bank & 3003 & & & & $11 / 85$ \\
\hline $\begin{array}{l}\text { EU Institutions } \\
\text { Total EU27 }\end{array}$ & $142208^{-}$ & $\begin{array}{r}758 \\
67940\end{array}$ & $\begin{array}{r}513 \\
53671\end{array}$ & $\begin{array}{l}6870 \\
9185\end{array}$ & $\begin{array}{r}8141 \\
273004\end{array}$ \\
\hline \multicolumn{6}{|l|}{ European Free Trade Association (EFTA) } \\
\hline Iceland & 132 & 168 & 374 & 37 & \\
\hline $\begin{array}{l}\text { Liechtenstein } \\
\text { Norway }\end{array}$ & $\begin{array}{r}25 \\
3114\end{array}$ & $\begin{array}{r}60 \\
2383\end{array}$ & 977 & 106 & $\begin{array}{r}83 \\
6580\end{array}$ \\
\hline Switzerland & 5214 & 8981 & 6965 & 96 & 21256 \\
\hline Total EFTA & 8485 & 11592 & 8312 & 241 & 28630 \\
\hline Other Europe & & & & & \\
\hline $\begin{array}{l}\text { Albania } \\
\text { Belarus }\end{array}$ & $\begin{array}{r}21 \\
120\end{array}$ & 12 & 2 & 1 & $\begin{array}{r}36 \\
135\end{array}$ \\
\hline $\begin{array}{l}\text { Belarus } \\
\text { Croatia }\end{array}$ & 173 & 82 & 23 & $\overline{3}$ & 281 \\
\hline Russia & 3595 & 1665 & 2049 & 23 & 7332 \\
\hline Turkey & 3248 & 1126 & 753 & 43 & 5170 \\
\hline Ukraine & 474 & 244 & 102 & 2 & 822 \\
\hline Serbia and Montenegro & 97 & 59 & -9 & 2 & 149 \\
\hline of which Serbia & 93 & 48 & -10 & 2 & 133 \\
\hline Other Montenegro & $810^{4}$ & $\begin{array}{r}11 \\
3322\end{array}$ & 4842 & 43 & 9016 \\
\hline Total Europe & 159231 & 86052 & 69750 & 9543 & 324576 \\
\hline \multicolumn{6}{|l|}{ Americas } \\
\hline $\begin{array}{l}\text { Argentina } \\
\text { Brazil }\end{array}$ & 357 & 233 & 277 & $\begin{array}{l}12 \\
35\end{array}$ & $\begin{array}{r}879 \\
456\end{array}$ \\
\hline $\begin{array}{l}\text { Brazil } \\
\text { Canada }\end{array}$ & 4.127 & 2936 & 4502 & 373 & $\begin{array}{r}4564 \\
11907\end{array}$ \\
\hline $\begin{array}{l}\text { Canada } \\
\text { Chile }\end{array}$ & 630 & $\begin{array}{l}250 \\
181\end{array}$ & $\begin{array}{l}40102 \\
945\end{array}$ & 17 & 1773 \\
\hline Colombia & 230 & 200 & $\begin{aligned} 245 \\
289\end{aligned}$ & 15 & $\begin{array}{l}1773 \\
734\end{array}$ \\
\hline Mexico & 952 & 421 & 770 & 44 & 2187 \\
\hline United States of America & 37925 & 34286 & 37348 & 2315 & 111874 \\
\hline Uruguay & 79 & 20 & & 1 & 107 \\
\hline Venezuela & 273 & 354 & 153 & 11 & 791 \\
\hline $\begin{array}{l}\text { Other Central American Countries } \\
\text { Other }\end{array}$ & 897 & 4211 & 8270 & 232 & 13610 \\
\hline $\begin{array}{l}\text { Other } \\
\text { Total Americas }\end{array}$ & $\begin{array}{r}394 \\
4881\end{array}$ & $\begin{array}{r}277 \\
43924\end{array}$ & 54082 & $\begin{array}{r}17 \\
3072\end{array}$ & 149159 \\
\hline \multicolumn{6}{|l|}{ Asia } \\
\hline China & 7611 & 2652 & 1084 & 39 & $\begin{array}{l}11386 \\
10892\end{array}$ \\
\hline $\begin{array}{l}\text { Hong Kong } \\
\text { India }\end{array}$ & $\begin{array}{l}4456 \\
4068\end{array}$ & $\begin{array}{l}1606 \\
2060\end{array}$ & $\begin{array}{l}4791 \\
1851\end{array}$ & $\begin{array}{l}39 \\
40\end{array}$ & $\begin{array}{r}10892 \\
8019\end{array}$ \\
\hline Indonesia & 461 & 325 & 420 & 17 & 1223 \\
\hline Iran & 307 & 128 & 43 & 2 & 480 \\
\hline $\begin{array}{l}\text { Israel } \\
\text { Japan }\end{array}$ & $\begin{array}{r}1387 \\
4334\end{array}$ & $\begin{array}{r}682 \\
415\end{array}$ & $\begin{array}{r}148 \\
3883\end{array}$ & 30 & 2247 \\
\hline $\begin{array}{l}\text { Japan } \\
\text { Malaysia }\end{array}$ & $\begin{array}{l}4334 \\
1272\end{array}$ & $\begin{array}{l}4315 \\
701\end{array}$ & $\begin{array}{r}3883 \\
895\end{array}$ & 125 & $\begin{array}{r}12657 \\
2897\end{array}$ \\
\hline $\begin{array}{l}\text { Malaysia } \\
\text { Pakistan }\end{array}$ & & 302 & 207 & ${ }_{9}^{20}$ & $\begin{array}{r}2897 \\
979\end{array}$ \\
\hline Philippines & $\begin{array}{l}461 \\
284\end{array}$ & 125 & 211 & 6 & 626 \\
\hline Saudi Arabia & 2488 & 2925 & 432 & 455 & $\begin{array}{r}0300 \\
6300\end{array}$ \\
\hline Singapore & 3447 & 3661 & 3053 & 39 & 10200 \\
\hline South Korea & 2344 & 1123 & 1533 & 23 & 5023 \\
\hline Taiwan & 1116 & 1145 & 850 & 8 & 3119 \\
\hline Thailand & $\begin{array}{l}1137 \\
1137\end{array}$ & 459 & 290 & 12 & 1898 \\
\hline $\begin{array}{l}\text { Residual Gulf Arabian Countries } \\
\text { Other Near \& Middle Fastern Countries }\end{array}$ & $\begin{array}{r}6590 \\
1316\end{array}$ & 4007 & 1037 & 189 & 11823 \\
\hline $\begin{array}{l}\text { Other Near \& Middle Eastern Countries } \\
\text { Other }\end{array}$ & 1316 & 610 & 1842 & 278 & 4046 \\
\hline Total Asia & 44291 & 28552 & 24838 & 1384 & 99065 \\
\hline \multicolumn{6}{|l|}{ Australasia \& Oceania } \\
\hline Australia & 3362 & 5025 & 7890 & 352 & 16629 \\
\hline New Zealand & 414 & 452 & 492 & 61 & 1419 \\
\hline $\begin{array}{l}\text { Other } \\
\text { Total Australasia \& Oceania }\end{array}$ & $\begin{array}{r}82 \\
3858\end{array}$ & $\begin{array}{r}208 \\
5685\end{array}$ & $\begin{array}{r}84 \\
8466\end{array}$ & $\begin{array}{r}10 \\
423\end{array}$ & $\begin{array}{r}384 \\
18432\end{array}$ \\
\hline Africa & & & & & \\
\hline & 1198 & 560 & 859 & 10 & 2627 \\
\hline Morocco & 565 & 137 & 27 & 8 & 737 \\
\hline $\begin{array}{l}\text { South Africa } \\
\text { Other North Africa }\end{array}$ & 2890 & 1723 & 2379 & 95 & $\begin{array}{l}7087 \\
1545\end{array}$ \\
\hline $\begin{array}{l}\text { Other North Africa } \\
\text { Other }\end{array}$ & 4693 & 3004 & 2582 & 60 & $\begin{array}{l}1545 \\
11239\end{array}$ \\
\hline Total Africa & $\begin{array}{r}4693 \\
10253\end{array}$ & 6828 & 5968 & 186 & 23235 \\
\hline International Organisations & - & 41 & 359 & - & 400 \\
\hline World Total & 265714 & 171082 & 163463 & 14608 & 614867 \\
\hline
\end{tabular}




\begin{tabular}{|c|c|c|c|}
\hline & $\begin{array}{r}\text { Trade in } \\
\text { goods }\end{array}$ & $\begin{array}{l}\text { Trade in } \\
\text { services }\end{array}$ & Income \\
\hline Debits & & & \\
\hline $\begin{array}{l}\text { Europe } \\
\text { European Union (EU) }\end{array}$ & & & \\
\hline Austria & 2633 & 987 & 687 \\
\hline Belgium & 17081 & 2319 & 1995 \\
\hline Bulgaria & 231 & 340 & 36 \\
\hline Cyprus & 106 & 881 & 614 \\
\hline Czech Republic & 3988 & 434 & 62 \\
\hline Denmark & 4117 & 1294 & 649 \\
\hline Estonia & 166 & 30 & 1 \\
\hline Finland & 2164 & 451 & 449 \\
\hline France & 21572 & 9636 & 11595 \\
\hline Germany & 46415 & 9127 & 13911 \\
\hline Greece & 701 & 1951 & 1869 \\
\hline Hungary & 3251 & 486 & 55 \\
\hline Ireland & 12873 & 4150 & 6907 \\
\hline Italy & 13940 & 4350 & 2518 \\
\hline Latvia & 440 & 94 & 13 \\
\hline Lithuania & 551 & 118 & 46 \\
\hline Luxembourg & 937 & 1411 & 6189 \\
\hline Malta & 170 & 326 & 117 \\
\hline Netherlands & 26449 & 3898 & 7857 \\
\hline Poland & 6106 & 1361 & 163 \\
\hline Portugal & 1749 & 1633 & 535 \\
\hline Romania & 1241 & 258 & 15 \\
\hline Slovak Republic & 1620 & 121 & 68 \\
\hline Slovenia & 357 & 48 & 27 \\
\hline Spain & 10381 & 9482 & 3287 \\
\hline Sweden & 6809 & 1614 & 1247 \\
\hline $\begin{array}{l}\text { European Central Bank } \\
\text { EU Institutions }\end{array}$ & - & - & - \\
\hline $\begin{array}{l}\text { EU Institutions } \\
\text { Total EU27 }\end{array}$ & $18604 \overline{8}$ & 56802 & $\begin{array}{r}2466 \\
63378\end{array}$ \\
\hline European Free Trade Association (EFTA) & & & \\
\hline Iceland & 433 & 56 & 503 \\
\hline $\begin{array}{l}\text { Liechtenstein } \\
\text { Norway }\end{array}$ & $\begin{array}{r}10 \\
21053\end{array}$ & $\begin{array}{r}20 \\
1092\end{array}$ & $\begin{array}{r}23 \\
1909\end{array}$ \\
\hline Switzerland & 7429 & 3215 & 4298 \\
\hline Total EFTA & 28925 & 4383 & 6733 \\
\hline Other Europe & & & \\
\hline Albania & $\overline{7}$ & 4 & - \\
\hline Belarus & 37 & 9 & 4 \\
\hline Croatia & 98 & 220 & 10 \\
\hline Russia & 5258 & 903 & 270 \\
\hline Turkey & 5351 & 1623 & 81 \\
\hline Ukraine & 283 & 217 & 91 \\
\hline Serbia and Montenegro & 94 & 49 & - \\
\hline of which Serbia & 93 & 38 & - \\
\hline Montenegro & 1 & 11 & $\overline{-}$ \\
\hline Other & 243 & 1279 & 8777 \\
\hline Total Europe & 226337 & 65489 & 79344 \\
\hline Americas & & & \\
\hline Argentina & 666 & 111 & 9 \\
\hline Brazil & 3111 & 447 & 79 \\
\hline Canada & 5770 & 1477 & 2321 \\
\hline Chile & 584 & 50 & 140 \\
\hline Colombia & 678 & 55 & 9 \\
\hline Mexico & 1032 & 398 & 35 \\
\hline United States of America & 26992 & 19200 & 42475 \\
\hline Uruguay & 103 & 8 & 4 \\
\hline Venezuela & 421 & 50 & 13 \\
\hline Other Central American Countries & 1146 & 2267 & -203 \\
\hline Other & 364 & 123 & 24 \\
\hline Total Americas & 40867 & 24186 & 44906 \\
\hline Asia & & & \\
\hline China & 30637 & 1180 & 279 \\
\hline Hong Kong & 8172 & 1056 & 2267 \\
\hline India & 5812 & 2292 & 204 \\
\hline Indonesia & 1388 & 119 & 20 \\
\hline Iran & 206 & 66 & 20 \\
\hline Israel & 1566 & 415 & 115 \\
\hline Japan & 8107 & 3627 & 5187 \\
\hline Malaysia & 1837 & 260 & 287 \\
\hline Pakistan & 809 & 443 & 28 \\
\hline Philippines & 525 & 217 & 15 \\
\hline Saudi Arabia & 766 & 249 & 559 \\
\hline Singapore & 4146 & 1803 & 1385 \\
\hline South Korea & 2563 & 351 & 258 \\
\hline 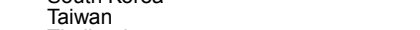 & 3118 & 247 & 78 \\
\hline Thailand & 2683 & 624 & 47 \\
\hline Residual Gulf Arabian Countries & 5387 & 1782 & 835 \\
\hline Other Near \& Middle Eastern Countries & 297 & 230 & 86 \\
\hline Other & 3905 & 1104 & 283 \\
\hline Total Asia & 81924 & 16065 & 11953 \\
\hline Australasia \& Oceania & & & \\
\hline Australia & 2315 & 2194 & 1327 \\
\hline New Zealand & 844 & 354 & 64 \\
\hline Other & 107 & 52 & -277 \\
\hline Total Australasia \& Oceania & 3266 & 2600 & 1114 \\
\hline Africa & & & \\
\hline Egypt & 659 & 681 & 79 \\
\hline Morocco & 343 & 248 & 13 \\
\hline South Africa & 4423 & 1101 & 1147 \\
\hline Other North Africa & 2851 & 432 & 182 \\
\hline Other & 3506 & 1472 & 395 \\
\hline Total Africa & 11782 & 3934 & 1816 \\
\hline International Organisations & - & 30 & 1291 \\
\hline World total & 364176 & 112304 & 140424 \\
\hline
\end{tabular}


Current account

continued

Summary transactions in 2010

\begin{tabular}{|c|c|c|c|}
\hline & $\begin{array}{r}\text { Trade in } \\
\text { goods }\end{array}$ & $\begin{array}{l}\text { Trade in } \\
\text { services }\end{array}$ & Income \\
\hline Balances & & & \\
\hline $\begin{array}{l}\text { Europe } \\
\text { European Union(EU) }\end{array}$ & & & \\
\hline Austria & -1170 & -344 & -286 \\
\hline Belgium & -3704 & 569 & 445 \\
\hline Bulgaria & 18 & -176 & -22 \\
\hline Cyprus & 456 & -279 & -544 \\
\hline Czech Republic & -2161 & 18 & -158 \\
\hline Denmark & -1360 & 1616 & 475 \\
\hline Estonia & 27 & 49 & 12 \\
\hline Finland & -666 & 823 & 172 \\
\hline France & -2344 & -723 & -1517 \\
\hline Germany & -18502 & 1673 & -5676 \\
\hline Greece & 680 & -832 & -1467 \\
\hline $\begin{array}{l}\text { Hungary } \\
\text { Ireland }\end{array}$ & -2169 & -14 & \\
\hline $\begin{array}{l}\text { Ireland } \\
\text { Italy }\end{array}$ & $\begin{array}{r}4059 \\
-5087\end{array}$ & $\begin{array}{r}4239 \\
801\end{array}$ & -12333 \\
\hline Latvia & $\begin{array}{r}-5087 \\
-272\end{array}$ & $\begin{array}{r}801 \\
-7\end{array}$ & $\begin{array}{r}1175 \\
-69\end{array}$ \\
\hline Lithuania & -326 & -22 & -32 \\
\hline Luxembourg & -691 & 733 & 5560 \\
\hline Malta & 223 & -99 & -52 \\
\hline Netherlands & -5199 & 6171 & 4168 \\
\hline Poland & -2302 & -229 & 406 \\
\hline Portugal & 88 & -854 & 47 \\
\hline Romania & -460 & 101 & 99 \\
\hline Slovak Republic & -1154 & 170 & -16 \\
\hline Slovenia & -137 & 44 & -33 \\
\hline Spain & -441 & -4343 & -128 \\
\hline Sweden & -1246 & 1297 & 1973 \\
\hline European Central Bank & - & - & \\
\hline EU Institutions & - & 756 & -1953 \\
\hline Total EU27 & -43840 & 11138 & -9707 \\
\hline European Free Trade Association (EFTA) & & & \\
\hline $\begin{array}{l}\text { Iceland } \\
\text { Liechtenstein }\end{array}$ & -301 & 112 & -129 \\
\hline $\begin{array}{l}\text { Liechtenstein } \\
\text { Norway }\end{array}$ & & 40 & -27 \\
\hline Switzerland & $\begin{array}{r}-17939 \\
-2215\end{array}$ & $\begin{array}{l}1291 \\
5766\end{array}$ & $\begin{array}{l}-932 \\
2667\end{array}$ \\
\hline Total EFTA & -20440 & 7209 & 1579 \\
\hline Other Europe & & & \\
\hline Albania & 21 & 8 & 2 \\
\hline Belarus & 83 & 1 & 1 \\
\hline Croatia & 75 & -138 & 13 \\
\hline Russia & -1663 & 762 & 1779 \\
\hline Turkey & -2103 & -497 & 672 \\
\hline Ukraine & 191 & 27 & 11 \\
\hline Serbia and Montenegro & 3 & 10 & -9 \\
\hline of which Serbia & - & 10 & -10 \\
\hline Montenegro & 3 & - & 1 \\
\hline $\begin{array}{c}\text { Other } \\
\end{array}$ & 567 & 2043 & -3935 \\
\hline Total Europe & -67106 & 20563 & -9594 \\
\hline Americas & & & \\
\hline Argentina & -309 & 122 & 268 \\
\hline Brazil & -894 & 389 & 1397 \\
\hline Canada & -1643 & 1428 & 2181 \\
\hline Chile & 46 & 131 & 805 \\
\hline Colombia & -448 & 145 & 280 \\
\hline Mexico & -80 & 23 & 735 \\
\hline USA & 10933 & 15086 & -5127 \\
\hline Uruguay & -24 & 12 & 3 \\
\hline Venezuela & -148 & 304 & 140 \\
\hline Other Central American Countries & -249 & 1944 & 8473 \\
\hline Other America & 30 & 154 & 21 \\
\hline Total Americas & 7214 & 19738 & 9176 \\
\hline Asia & & & \\
\hline China & -23026 & 1472 & 805 \\
\hline Hong Kong & -3716 & 550 & 2524 \\
\hline India & -1744 & -232 & 1647 \\
\hline Indonesia & -927 & 206 & 400 \\
\hline Iran & 101 & 62 & 23 \\
\hline Israel & -179 & 267 & 33 \\
\hline Japan & -3773 & 688 & -1304 \\
\hline Malaysia & -565 & 441 & 608 \\
\hline Pakistan & -348 & -141 & 179 \\
\hline Philippines & -241 & $\begin{array}{r}-141 \\
-92\end{array}$ & 196 \\
\hline Saudi Arabia & 1722 & 2676 & -127 \\
\hline Singapore & -699 & 1858 & 1668 \\
\hline South Korea & -219 & 772 & 1275 \\
\hline Taiwan & -2002 & 898 & 772 \\
\hline Thailand & -1546 & -165 & 243 \\
\hline Residual Gulf Arabian Countries & 1203 & 2225 & 202 \\
\hline Other Near \& Middle Eastern Countries & 1019 & 380 & 1756 \\
\hline Other & -2693 & 622 & 1985 \\
\hline Total Asia & -37633 & 12487 & 12885 \\
\hline Australasia \& Oceania & & & \\
\hline Australia & 1047 & 2831 & 6563 \\
\hline New Zealand & -430 & 98 & 428 \\
\hline Other & -25 & 156 & 361 \\
\hline Total Australasia \& Oceania & 592 & 3085 & 7352 \\
\hline Africa & & & \\
\hline Egypt & 539 & -121 & 780 \\
\hline Morocco & 222 & -111 & 14 \\
\hline South Africa & -1533 & 622 & 1232 \\
\hline Other North Africa & -1944 & 72 & -61 \\
\hline Other & 1187 & 2432 & 2187 \\
\hline Total Africa & -1529 & 2894 & 4152 \\
\hline International Organisations & - & 11 & -932 \\
\hline World total & -98462 & 58778 & 23039 \\
\hline
\end{tabular}




\begin{tabular}{|c|c|}
\hline & \\
\hline & \\
\hline Credits & \\
\hline $\begin{array}{l}\text { Europe } \\
\text { European Union (EU) }\end{array}$ & \\
\hline Austria & CUGP \\
\hline Belgium $^{1}$ & $\mathrm{AA} 2 \mathrm{Q}$ \\
\hline Bulgaria & ZWVI \\
\hline $\begin{array}{l}\text { Cyprus } \\
\text { Cys }\end{array}$ & AA2R \\
\hline Czech Republic & LEPQ \\
\hline Denmark & $\mathrm{LEQR}$ \\
\hline Estonia & $\mathrm{zWVTK}$ \\
\hline Finland & LEUD \\
\hline France & LEUM \\
\hline Germany & LEQI \\
\hline Greece & LEUV \\
\hline Hungary & BFKO \\
\hline Ireland & BFLV \\
\hline Italy & BFOD \\
\hline Latvia & ZWVM \\
\hline Lithuania & zWVN \\
\hline Luxembourg ${ }^{1}$ & $\mathrm{AA} 2 \mathrm{U}$ \\
\hline Malta & $\mathrm{AA} 2 \mathrm{~V}$ \\
\hline Netherlands & $\mathrm{BFQF}$ \\
\hline Poland & BFRY \\
\hline Portugal & BFSH \\
\hline Romania & zWvo \\
\hline Slovak Republic & ZWVP \\
\hline Slovenia & zWvQ \\
\hline Spain & LEST \\
\hline Sweden & BFTI \\
\hline European Central Bank & ZWVF \\
\hline $\begin{array}{l}\text { EU Institutions } \\
\text { Total EU27 }\end{array}$ & $\begin{array}{l}\text { CSFH } \\
\text { G970 }\end{array}$ \\
\hline Euronean Free Trade Association (FFTA) & \\
\hline $\begin{array}{l}\text { European Free Trade Association (EFTA) } \\
\text { Iceland }\end{array}$ & \\
\hline Liechtenstein & BFPE \\
\hline Norway & $\mathrm{BFQO}$ \\
\hline Switzerland & LEOY \\
\hline Total EFTA & CTFQ \\
\hline Other Europe & \\
\hline Albania & zWVG \\
\hline Belarus & zWVH \\
\hline Croatia & $\mathrm{zWVU}$ \\
\hline Russia & BFSQ \\
\hline Turkey & BFUŨ \\
\hline Ukraine & ZWVR \\
\hline Serbia and Montenegro ${ }^{2}$ & BFWC \\
\hline of which Serbia & KNC9 \\
\hline Montenegro & KND2 \\
\hline Other & LEVW \\
\hline Total Europe & LERA \\
\hline Americas & \\
\hline Argentina & zWVT \\
\hline Brazil & LENO \\
\hline Canada & LEOP \\
\hline Chile & zWVU \\
\hline Colombia & zWvV \\
\hline Mexico & BFPN \\
\hline United States of America & BFVB \\
\hline Uruguay & $\mathrm{zWVW}$ \\
\hline Venezuela & $\mathrm{zwvx}$ \\
\hline Other Central American Countries & JISS \\
\hline Other & LEVE \\
\hline Total Americas & LESK \\
\hline Asia & \\
\hline & LEPH \\
\hline Hong Kong & BFJR \\
\hline India & BFMY \\
\hline Indonesia & BFKX \\
\hline Iran & zWWA \\
\hline Israel & BFMP \\
\hline Japan & BFOM \\
\hline Malaysia & BFPW \\
\hline Pakistan & BFRP \\
\hline Philippines & BFRG \\
\hline Saudi Arabia & BFSZ \\
\hline Singapore & BFTR \\
\hline South Korea & BFOV \\
\hline Taiwan & BFUS \\
\hline Thailand & BFUA \\
\hline Residual Gulf $A$ & JITT \\
\hline Other Near \& Middle Eastern Countries & ZWWC \\
\hline Other & LEWF \\
\hline Total Asia & LETC \\
\hline Australasia \& Oceania & \\
\hline & CWBG \\
\hline New Zeal & $\mathrm{BFQX}$ \\
\hline Other & LEVN \\
\hline Total Australasia \& Oceania & LETU \\
\hline Africa & \\
\hline Egypt & \\
\hline Morocco & ZWWF \\
\hline South Africa & BFWU \\
\hline Other North Africa & JIRU \\
\hline Other & LEWO \\
\hline Total Africa & LERS \\
\hline International Organisations & CTEY \\
\hline World total & HBOE \\
\hline
\end{tabular}

1 Before 1999 only combined BLEU data is available under identifier CTFH. 2 Separate data for Serbia and Montenegro is available from 2007. 
Europe

European Union (EU)

Austria
Belgium

Bulgaria

Cyprus

Denmark

Estonia

France

Germany

Hungary

Ireland

Italy

Lithuania

Malta

Netherlands

Portuga

Romania

Slovak Republic

Slovenia

Spain

European Central Bank

EU Institutions

$$
\text { Iceland }
$$

Liechtenstein

Norway

Switzerland
Total EFTA

Other Europe

Albania

Belarus

Russia

Turkey

Serbia and Montenegro ${ }^{2}$

of which Serbia

Other

Other
Total Europe

\section{Americas}

Argentina

Brazil

Canada

Chile

Colombia

Mexico

Uruguay

ther Central American Countries

Other

Total Americas

Asia

China

Hong Kong

Indonesia

Iran

Israel

Japan

Makistan

Philippines

Saudi Arabia

South Korea

Taiwan

Residual Gulf Arabian Countries

Other Near \& Middle Eastern Countries

Other
Total Asia

Australasia \& Oceania

Australia

Other

Total Australasia \& Oceania

Africa

Egypt

Morocco

Other North Africa

Other

Other
Total Africa

International Organisations

World total

\begin{tabular}{|c|c|c|c|c|c|c|c|c|c|c|c|}
\hline CUGW & 2508 & 3108 & 3801 & 4093 & 3762 & 4161 & 4793 & 4678 & 4397 & 4035 & 4348 \\
\hline $\mathrm{AA} 34$ & 14129 & 15315 & 16184 & 16265 & 17055 & 19383 & 21069 & 22289 & 23819 & 19780 & 21478 \\
\hline ZWWL & 191 & 187 & 252 & 248 & 339 & 464 & 508 & 664 & 672 & 485 & 614 \\
\hline $\mathrm{AA} 35$ & 1267 & 1481 & 1382 & 1479 & 1463 & 1721 & 3242 & 1782 & 1801 & 522 & \\
\hline LEPR & 1107 & 1420 & 1592 & 1846 & 1810 & 2462 & 3698 & 3660 & 4288 & 3801 & 4505 \\
\hline LEQS & 4449 & 4536 & 5248 & 5204 & 5383 & 6837 & 8996 & 6188 & 6668 & 6064 & 6116 \\
\hline zWWNN & 376 & 318 & 342 & 302 & 433 & 425 & 2198 & 312 & 220 & 168 & 203 \\
\hline LEUE & 3568 & 3708 & 3349 & 3310 & 2951 & 3297 & 4023 & 3853 & 4048 & 3038 & 3084 \\
\hline UN & 34140 & 35012 & 34493 & 36719 & 38960 & 44350 & 51895 & 48934 & 48546 & 48506 & \\
\hline LEQJ & 44988 & 44176 & 44582 & 49808 & 54128 & 63807 & 70259 & 78723 & 74991 & 58373 & 70 \\
\hline LEŨW & 3012 & 3129 & 3045 & 3156 & 3301 & 3853 & 4449 & 5154 & 5296 & 4816 & 46 \\
\hline BFKP & 865 & 884 & 1039 & 1301 & 1799 & 2183 & 2760 & 2871 & 3011 & 3045 & 38 \\
\hline BFLW & 15997 & 18999 & 20654 & 17968 & 19908 & 23546 & 26320 & 30929 & 33398 & 26070 & 246 \\
\hline BFOE & 15421 & 16030 & 15756 & 17255 & 18364 & 20064 & 20464 & 22087 & 228 & 184 & \\
\hline ZWWP & 467 & 462 & 507 & 551 & 737 & 784 & 897 & 740 & 506 & 414 & \\
\hline zWWQ & 286 & 272 & 316 & 313 & ñ & 37 & 402 & 450 & 485 & & \\
\hline AA38 & 3089 & 3581 & 3603 & 3923 & 4938 & 5483 & 8780 & 8334 & 9813 & 6268 & \\
\hline AA3 9 & 412 & 462 & 469 & 515 & 556 & 584 & 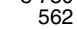 & 707 & 872 & 615 & \\
\hline $\mathrm{BFQG}$ & 30793 & 30662 & 26445 & 27264 & 29790 & 33579 & 43477 & 49257 & 36964 & 30616 & 384 \\
\hline BFRZ & 1340 & 1589 & 1620 & 1929 & 2349 & 3301 & 5166 & 550 & 6187 & & 7 \\
\hline BFSI & 3049 & 3076 & 3289 & 3729 & 376 & 3962 & 52 & 4051 & 4518 & 36 & \\
\hline ZWWR & $\begin{array}{r}464 \\
\end{array}$ & 565 & 655 & 795 & 950 & 1023 & 1066 & 11 & 1072 & & \\
\hline ZWWS & 21 & 208 & 253 & 292 & 31 & 500 & 11 & 1468 & 1817 & & \\
\hline ZWWT & 198 & 206 & 238 & 223 & 23 & 277 & 6 & & 416 & & \\
\hline $\mathrm{L}$ & 14031 & 1623 & 18502 & 20164 & 20114 & 25120 & 27708 & 27433 & 26958 & 2504 & 233 \\
\hline BFTJ & 7023 & 西 & 6506 & 6748 & 7325 & 8112 & 9382 & 8830 & 10556 & & \\
\hline ZWWI & & & & & & & & & & & \\
\hline CSFI & 12211 & 11325 & 11789 & 12997 & 13153 & 15107 & 15395 & 15841 & 16407 & 17447 & 18 \\
\hline
\end{tabular}

$\begin{array}{rrrrrrrrrrr}12211 & 11325 & 11789 & 12997 & 13153 & 15107 & 15395 & 15841 & 16407 & 17447 & 18455 \\ 215808 & 223848 & 225912 & 238397 & 254180 & 294762 & 344742 & 356316 & 350580 & 300956 & 325383\end{array}$

$\begin{array}{rrrrrrrrrrr}463 & 347 & 359 & 374 & 474 & 523 & 813 & 935 & -741 & 790 & 998 \\ 147 & 126 & 79 & 94 & 76 & 97 & 153 & 208 & 165 & 68 & 55 \\ 6920 & 7103 & 7497 & 8097 & 10357 & 14830 & 17905 & 18503 & 25391 & 19375 & 24172 \\ 18877 & 16179 & 13203 & 11769 & 11537 & 15377 & 19041 & 20294 & 4262 & 9911 & 15101 \\ 26407 & 23755 & 21138 & 20334 & 22444 & 30827 & 37912 & 39940 & 29077 & 30144 & 40326\end{array}$

$\begin{array}{rrrrrrrrrrr}41 & 28 & 26 & 24 & 27 & 15 & 20 & 35 & 32 & 23 & 17 \\ 66 & 37 & 46 & 31 & 77 & 290 & 758 & 633 & 145 & 29 & 50 \\ 136 & 159 & 155 & 152 & 168 & 250 & 320 & 331 & 378 & 296 & 339 \\ 2214 & 2734 & 2564 & 3119 & 4514 & 6946 & 9130 & 9258 & 10024 & 6334 & 6505 \\ 2131 & 2478 & 3077 & 3564 & 4257 & 4795 & 5342 & 6144 & 6765 & 6152 & 7109 \\ 133 & 158 & 196 & 195 & 285 & 380 & 465 & 710 & 710 & 396 & 618 \\ 141 & 110 & 100 & 110 & 108 & 100 & 194 & 208 & 205 & 177 & 186 \\ \cdot & \cdot & \cdot & \cdot & \cdot & \cdot & \cdot & 198 & 191 & 159 & 170 \\ 8 . & . & . & . & . & . & 10 & 14 & 18 & 16 \\ 8493 & 9091 & 8227 & 7290 & 9040 & 12268 & 15411 & 22499 & 24585 & 11792 & 10566\end{array}$ $\begin{array}{rrrrrrrrrrr}849 \dot{3} & 909 \dot{1} & 822 \dot{7} & 7290 & 9040 & 12268 & 1541 \dot{1} & 22499 & 24585 & 11792 & 10566 \\ 255570 & 262398 & 261441 & 273216 & 295100 & 350633 & 414294 & 436074 & 422501 & 356299 & 391099\end{array}$ $\begin{array}{rrrrrrrrrrr}367 & 360 & 315 & 312 & 345 & 381 & 495 & 533 & 733 & 793 & 804 \\ 1533 & 1734 & 1770 & 1801 & 1926 & 2257 & 2447 & 2551 & 3101 & 2967 & 3701 \\ 6862 & 6283 & 6519 & 6396 & 7745 & 8027 & 10006 & 9330 & 7117 & 8464 & 10143 \\ 530 & 574 & 560 & 499 & 600 & 623 & 657 & 674 & 753 & 743 & 795 \\ 338 & 421 & 290 & 279 & 326 & 378 & 424 & 463 & 791 & 670 & 764 \\ 1154 & 1349 & 927 & 870 & 857 & 872 & 934 & 1062 & 1386 & 1084 & 1511 \\ 70754 & 77600 & 70370 & 69938 & 71386 & 82569 & 104975 & 114563 & 101619 & 82621 & 91480 \\ 63 & 55 & 73 & 56 & 54 & 72 & 94 & 96 & 130 & 146 & 117 \\ 279 & 248 & 255 & 176 & 265 & 468 & 743 & 634 & 742 & 506 & 496 \\ 7222 & 6382 & 5046 & 5779 & 7115 & 10999 & 16344 & 18143 & 16864 & 9763 & 3773 \\ 892 & 701 & 567 & 476 & 475 & 548 & 1202 & -3036 & 19646 & 9058 & 579 \\ 89994 & 95707 & 86992 & 86582 & 91094 & 107194 & 138321 & 145013 & 152882 & 116815 & 114163\end{array}$

$\begin{array}{rrrrrrrrrrr}5692 & 6739 & 7774 & 9279 & 11557 & 14428 & 16945 & 20882 & 25513 & 26217 & 32304 \\ 10248 & 9901 & 8323 & 7703 & 8111 & 9392 & 11049 & 12046 & 13463 & 11497 & 11684\end{array}$

$\begin{array}{rrrrrrrrrrr}10248 & 9901 & 8323 & 7703 & 8111 & 9392 & 11049 & 12046 & 13463 & 11497 & 11684 \\ 3172 & 3536 & 3420 & 3668 & 4398 & 5509 & 6589 & 7395 & 8272 & 7794 & 9314 \\ 1430 & 1482 & 1350 & 1394 & 1222 & 1266 & 1399 & 1291 & 1510 & 1509 & 1659\end{array}$

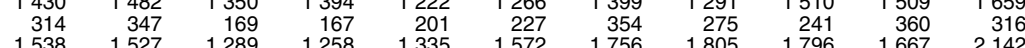

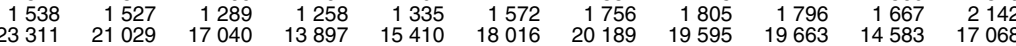

$\begin{array}{rrrrrrrrrrr}23311 & 21029 & 17040 & 13897 & 15410 & 18016 & 20189 & 19595 & 19663 & 14583 & 17068 \\ 2966 & 2488 & 2133 & 2193 & 2440 & 2356 & 2709 & 2587 & 2701 & 2460 & 2459\end{array}$

$\begin{array}{lllllllllll}2966 & 2488 & 2133 & 2193 & 2440 & 2356 & 2709 & 2587 & 2701 & 2460 & 2459 \\ 879 & 936 & 1176 & 1166 & 1243 & 1250 & 1364 & 1292 & 1411 & 1431 & 1613\end{array}$

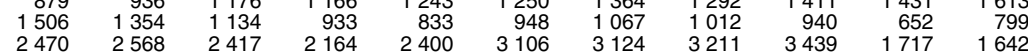

$\begin{array}{lllllllllll}5654 & 5202 & 4342 & 4668 & 5705 & 7198 & 8090 & 9272 & 9116 & 7110 & 7494\end{array}$

$\begin{array}{lllllllllll}3694 & 3147 & 3034 & 2776 & 3184 & 3627 & 3740 & 3808 & 4416 & 3489 & 3196 \\ 3956 & 3211 & 2744 & 2541 & 2767 & 2861 & 2960 & 3219 & 3301 & 2653 & 3451\end{array}$

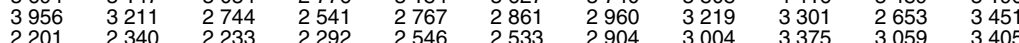

$\begin{array}{rrrrrrrrrrr}2201 & 2340 & 2233 & 2292 & 2546 & 2533 & 2904 & 3004 & 3375 & 3059 & 3405 \\ 3001 & 3280 & 2764 & 3133 & 3710 & 4494 & 5734 & 6297 & 6929 & 6015 & 8396 \\ 577 & 569 & 540 & 515 & 514 & 704 & 1371 & 942 & 977 & 756 & 716\end{array}$

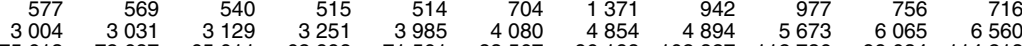

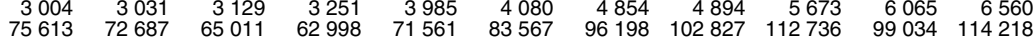

$\begin{array}{rrrrrrrrrrr}3617 & 3786 & 4312 & 4756 & 4950 & 6184 & 6368 & 7377 & 7282 & 7668 & 6396 \\ 974 & 1016 & 1020 & 1045 & 1184 & 1290 & 1303 & 1296 & 1355 & 1266 & 1388 \\ 193 & 191 & 197 & 206 & 232 & 210 & 575 & 193 & 267 & 250 & -886\end{array}$

$\begin{array}{rrrrrrrrrrr}193 & 191 & 197 & 206 & 232 & 210 & 575 & 193 & 267 & 250 & -88 \\ 4784 & 4993 & 5529 & 6007 & 6366 & 7684 & 8246 & 8866 & 8904 & 9184 & 7696\end{array}$

$\begin{array}{lllllllllll}1049 & 1070 & 913 & 932 & 1012 & 1198 & 1682 & 1504 & 1681 & 1514 & 1460\end{array}$

$\begin{array}{rrrrrrrrrrr}609 & 630 & 624 & 608 & 685 & 627 & 712 & 877 & 868 & 614 & 619 \\ 4132 & 4517 & 4437 & 5017 & 5418 & 6448 & 6646 & 5720 & 7297 & 6233 & 7142 \\ 1266 & 1077 & 1007 & 977 & 1219 & 1633 & 2561 & 2806 & 3412 & 2406 & 3484 \\ 4187 & 5416 & 5375 & 6217 & 6292 & 6611 & 7180 & 8391 & 9078 & 8157 & 8365\end{array}$

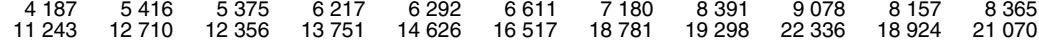

$\begin{array}{lllllllllll}1756 & 1793 & 1724 & 1265 & 1833 & 2153 & 2963 & 3255 & 3469 & 3201 & 3347\end{array}$

HBOF $\quad 438960 \quad 450288 \quad 432753 \quad 443819 \quad 480580 \quad 567748 \quad 678803 \quad 715333 \quad 722828 \quad 603457 \quad 651593$ 
9.2 curentatcosomt

continued

2000

$2001 \quad 2002$

$£$ million

Balances

Europe

European Union (EU)

Austria
Belgium

Bulgaria
Cyprus

Czech Republic

Denmark

Finland

France

Greece

Hungary

Ireland
Italy
Latvia

Latvia

Luxembourg

Malta

Poland

Portugal

Romania

Slovenia

Spain

European Central Bank

EU Institutions

uropean Free Trade Association (EFTA)

celand

Norway

Switzerland
Total EFTA

Other Europe

Albania

Belarus

Croatia
Russia

Turkey

Serbia and Montenegro

of which Serbia

Other

Total Europe

Americas

Argentina

Brazil

Chile

Colomb

United States of Americ

Uruguay

Other Central American Countries

Other

Total Americas

Asia

China

Hong Kong

India

Iran

Israel

Malaysia

Pakistan

Philippines

Saudi Arabia

Singapore
South Korea

Taiwan

Residual Gulf Arabian Countries Other Near \& Middle Eastern Countries Other

Total Asia

Australasia \& Oceania

Australia

Other

Total Australasia \& Oceania

Africa

Egypt

South Africa

Other North Africa

Other

Total Africa

International Organisations

World total

\begin{tabular}{|c|c|}
\hline CUGX & -187 \\
\hline $\mathrm{AA} 4 \mathrm{H}$ & 598 \\
\hline zwXo & -25 \\
\hline AA4 I & \\
\hline LEPS & 118 \\
\hline $\mathrm{LEQT}$ & 176 \\
\hline zWX̃Q & -266 \\
\hline LEUF & -534 \\
\hline LEUO & -2311 \\
\hline LEQK & -4996 \\
\hline LEUX & 95 \\
\hline BFKQ & 407 \\
\hline BFLX & 3164 \\
\hline BFOF & 2226 \\
\hline zwXs & -349 \\
\hline ZWXT & -129 \\
\hline AA4L & 1273 \\
\hline AA4M & -92 \\
\hline $\mathrm{BFQH}$ & 2068 \\
\hline BFSA & 392 \\
\hline BFSU & -258 \\
\hline zWXu & -21 \\
\hline zWXV & 30 \\
\hline zWXW & 31 \\
\hline LESV & -818 \\
\hline BFTK & 796 \\
\hline ZWXL & \\
\hline CSFU & 170 \\
\hline$G 9$ & \\
\hline
\end{tabular}

$\begin{array}{rrr}-762 & -1395 \\ -703 & -1179 \\ 61 & 36\end{array}$

61

$\begin{array}{r}39 \\ 132\end{array}-222$

$\begin{array}{rr}132 & -582 \\ -213 & -215 \\ -552 & -473\end{array}$

$\begin{array}{lll}-677 & -2431 & -445\end{array}$

-2431
-5072

$\begin{array}{rr}-5072 & -12462 \\ -325 & -262\end{array}$

$\begin{array}{rrrrr}-5294 & -6532 & -508 & -383 \\ -662 & -14258 & -19420 & -18699 & -25145\end{array}$

\begin{tabular}{rrrrrrrr}
-262 & -14258 & -19420 & -18669 & -25145 & -2835 & -13028 & -4679 \\
113 & -321 & -660 & -1186 & -1419 & -915 & -11163 & -22783 \\
\hline & -105 & -373 & -1293 & -1189 & -121 & -1489 & -1634
\end{tabular}

$\begin{array}{llllllllll}-298 & -325 & 262 & -321 & -660 & -1186 & -1419 & -915 & -1268 & -1634 \\ 259 & 289 & 113 & -105 & -373 & -1293 & -1189 & -1221 & -1489 & -2145\end{array}$

$\begin{array}{rrrrrrrrrr}3369 & 3127 & 4550 & 5604 & 7346 & -8337 & 9813 & -9702 & 3939 & -4541 \\ 1741 & 685 & -632 & -1725 & -2332 & -1305 & -1310 & -2159 & -865 & -3160\end{array}$

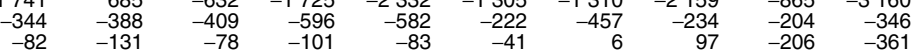

$\begin{array}{rrrrrrrrrr}-82 & -131 & -78 & -101 & -83 & -41 & 6 & 97 & -206 & -361 \\ 1064 & 540 & -120 & 144 & 1861 & 4484 & 5585 & 10643 & 10098 & 5609 \\ -139 & -134 & -132 & 273 & -228 & -294 & -226 & -133 & 426 & 43\end{array}$

$\begin{array}{rrrrrrrrrr}-139 & -134 & -132 & 273 & -228 & -294 & -226 & -133 & 426 & 43 \\ 5756 & 8372 & 7221 & -975 & -2111 & -3081 & -4793 & 15137 & 12341 & 5278 \\ 377 & 436 & 348 & 55 & -298 & -691 & -1517 & -1298 & -1331 & -2148\end{array}$

$\begin{array}{rrrrrrrrrr}377 & 436 & 348 & 55 & -298 & -691 & -1517 & -1298 & -1331 & -2148 \\ -193 & -446 & -911 & -683 & -642 & -1348 & -762 & -782 & -556 & -759\end{array}$

$\begin{array}{rrrrrrrrrr}-193 & -446 & -911 & -683 & -642 & -1348 & -762 & -782 & -556 & -759 \\ -75 & -76 & -94 & -86 & -173 & -177 & -91 & 209 & 79 & -270 \\ 67 & 11 & 6 & -19 & -77 & -625 & -795 & -961 & -1092 & -994\end{array}$

$\begin{array}{rrrrrrrrrr}67 & 11 & 6 & -19 & -77 & -625 & -795 & -961 & -1092 & -994 \\ 90 & 64 & 68 & 78 & -8 & -527 & -76 & -47 & -23 & -127\end{array}$

$\begin{array}{rrrrrrrrrr}1083 & -5243 & -5952 & -4317 & -5593 & -3933 & -3673 & -1703 & -4247 & -4988 \\ 11 & 2 & 623 & 1121 & 1039 & 1060 & 2530 & 2298 & 2206 & 2062\end{array}$

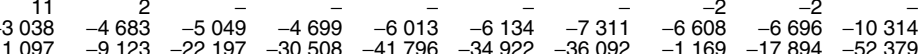

$\begin{array}{llllllllllll}\text { BFNJ } & -129 & -61 & -94 & -67 & -126 & -55 & -175 & 477 & 2349 & -22 & -287\end{array}$

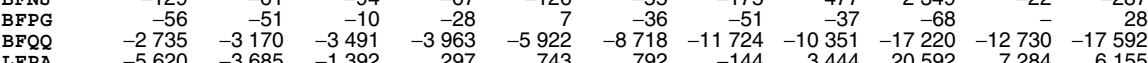

$\begin{array}{lrlllllllrrr}\text { LEPA } & -5620 & -3685 & -1392 & 297 & 743 & 792 & -144 & 3444 & 20592 & 7284 & 6155 \\ \text { CTFS } & -8540 & -6967 & -4987 & -3761 & -5298 & -8017 & -12093 & -6467 & 5653 & -5468 & -11696\end{array}$

$\begin{array}{lr}\text { ZWXM } & -32 \\ \text { ZWXN } & -3 \\ \text { ZWXP } & -8 \\ \text { BFSS } & -832 \\ \text { BFUL } & 583 \\ \text { ZWXX } & 63 \\ \text { BFWE } & -3\end{array}$
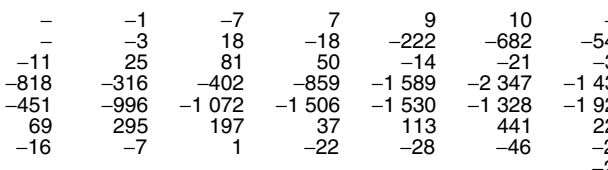

-3
-542
-37
-1436
-1920
227
-23
-27

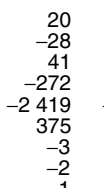

$\begin{array}{rr}14 & 19 \\ 60 & 85 \\ 87 & -58 \\ -70 & 827 \\ -2027 & -1939 \\ 519 & 204 \\ -6 & -37 \\ -7 & -37\end{array}$

$\begin{array}{llllllllllll} & -3561 & -4764 & -3586 & -937 & -878 & -2158 & -2530 & -8602 & -13100 & -4034 & -1549\end{array}$

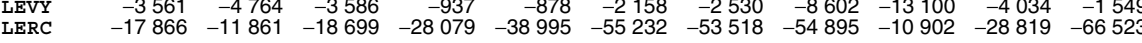

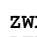

604
138
422
-55
152
263
8358
17
-59
771
-413
10198

472
142
1079
-138
71
-47
4526
11
361
1934
-290

$\begin{array}{rr}7 & 97 \\ 108 & -143 \\ 42 & 580 \\ -111 & 18 \\ 129 & 210 \\ 693 & 520 \\ 8679 & 11646 \\ -18 & 46 \\ 344 & 162 \\ 3818 & 3394 \\ -178 & -67\end{array}$

236
-40
-172
464
286
871
199
-14
299
3228
294

172
72
454
828
266

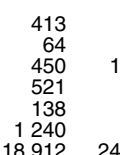

282
289
1302
541
-6

$\begin{array}{rrr}107 & -16 & 75 \\ 529 & 1149 & 863 \\ -924 & -1404 & 1764\end{array}$

BFPP

ZWYC

JISU

LEVG

$\begin{array}{rrrrrrrrrr}1934 & 3818 & 3394 & 3228 & 3795 & 3600 & 4638 & 4505 & 4521 & 9837 \\ -290 & -178 & -67 & 294 & -369 & 154 & 3020 & -18192 & -8312 & 154 \\ 8121 & 13513 & 16463 & 20651 & 28204 & 25431 & 35723 & 7839 & 18375 & 34996\end{array}$

-1
-60

$-60-17$

\begin{tabular}{rrrr}
999 & 458 & -228 & -30 \\
\hline 29 & 676
\end{tabular}

$\begin{array}{rrrr}863 & 21332 & 21598 & 20394\end{array}$

$\begin{array}{lllllllllll}-34465 & -3881 & -5119 & -6038 & -7278 & -9361 & -11256 & -14237 & -16705 & -17543 & -20 \\ -4 & 918\end{array}$

LEPJ

BFJT

BFNA
BFKZ
ZWYG

ZWYG

BFOO

BFPY

BFRI
BFTB
BFTT

BFTT

BFUU

BFUC

JITV

LEWH

$\begin{array}{lllll}-4832 & -4659 & -3452 & -2234 & -111\end{array}$

$\begin{array}{lll}-68 & -581 & -38 \\ -658 & -733 & -630\end{array}$

$\begin{array}{rrr}163 & -733 & -630 \\ 500 & 434 & 457\end{array}$

$\begin{array}{rrr}500 & 434 & 641 \\ -7080 & -6159 & -4589\end{array}$

$\begin{array}{rrr}-1 & -658 & -249 \\ -371 & -302 & -584 \\ -867 & -622 & -527\end{array}$

$\begin{array}{rrr}-867 & -622 & -527 \\ 2254 & 1837 & 1643 \\ -156 & 232 & 179\end{array}$

$\begin{array}{rrr}-1520 & -947 & -342 \\ -2396 & -1739 & -1174\end{array}$

$\begin{array}{rrr}-1200 & -1271 & -1251 \\ 2385 & 1841 & 2092\end{array}$

$525-730$

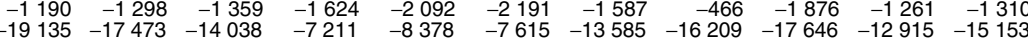

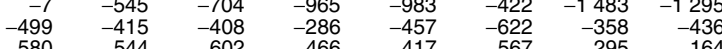

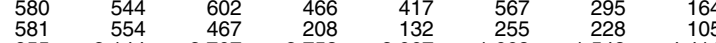

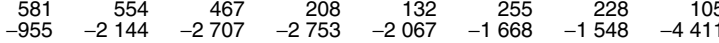

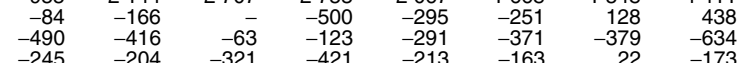

$\begin{array}{rrrrrrrr}-490 & -416 & -63 & -123 & -291 & -371 & -379 & -634 \\ -245 & -204 & -321 & -421 & -213 & -163 & 22 & -173 \\ 2663 & 2452 & 1578 & 2059 & 1927 & 1801 & 3865 & 4658 \\ 205 & 428 & 1003 & 1033 & -887 & 966 & 2762 & 2706\end{array}$

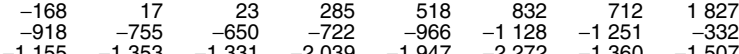

$\begin{array}{rrrrrrrr}-1155 & -1353 & -1331 & -2039 & -1947 & -2272 & -1360 & -1507 \\ 2423 & 3058 & 6008 & 3606 & 3519 & 5216 & 5263 & 3427\end{array}$

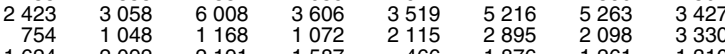

$\begin{array}{lrrrrrrrrrrr}\text { CWJK } & 3338 & 3097 & 2629 & 2296 & 4032 & 3737 & 4203 & 4818 & 5687 & 5724 & 10233 \\ \text { BFQZ } & 17 & 83 & 184 & 241 & 68 & 79 & 92 & 117 & 16 & -52 & 31 \\ \text { LEVP } & -38 & -40 & -7 & 11 & -13 & -27 & -353 & 37 & 57 & 67 & 472 \\ \text { LETW } & 3317 & 3140 & 2806 & 2548 & 4087 & 3789 & 3942 & 4972 & 5760 & 5739 & 10736 \\ & & & & & & & & & & & \\ \text { ZWYK } & - & -16 & 100 & 30 & 182 & 63 & -173 & 391 & 517 & 637 & 1167 \\ \text { ZWYL } & -111 & -150 & -205 & -183 & -278 & -304 & -278 & -455 & -224 & -114 & 118 \\ \text { BFWW } & -465 & -441 & 271 & -168 & 635 & 1065 & -748 & 848 & -1195 & -862 & -55 \\ \text { JIRW } & -479 & -294 & -342 & 14 & -314 & -4124 & -1334 & -1643 & -1657 & -873 & -1939 \\ \text { LEWQ } & -33 & -873 & -643 & -1068 & -1150 & 3057 & -495 & -976 & 703 & 1280 & 2874 \\ \text { LERU } & -1088 & -1774 & -819 & -1375 & -925 & -243 & -3028 & -1835 & -1856 & 68 & 2165 \\ \text { CTFA } & -1255 & -1215 & -1164 & -726 & -1330 & -1570 & -2337 & -2582 & -2948 & -2764 & -2947 \\ \text { HBOG } & -25829 & -21062 & -18401 & -18380 & -24890 & -32667 & -43095 & -34826 & -19753 & -20316 & -36726\end{array}$

1 Before 1999 only combined BLEU data is available under identifier CTFJ. 2 Separate data for Serbia and Montenegro is available from 2007. 


\begin{tabular}{|c|c|c|c|c|c|c|c|c|c|c|c|c|}
\hline & & & & & & & & & & & & $£$ million \\
\hline & & 2000 & 2001 & 2002 & 2003 & 2004 & 2005 & 2006 & 2007 & 2008 & 2009 & 2010 \\
\hline Exports & & & & & & & & & & & & \\
\hline $\begin{array}{l}\text { Europe } \\
\text { European }\end{array}$ & & & & & & & & & & & & \\
\hline $\begin{array}{l}\text { European U } \\
\text { Austria }\end{array}$ & LGHT & 1549 & 1632 & 1688 & 1686 & 1528 & 1851 & 2242 & 2080 & 2256 & 2034 & 2106 \\
\hline Belgium & $\triangle 7 \mathrm{PS}$ & 12176 & 11657 & 12567 & 13627 & 13212 & 13999 & 15711 & 14788 & 16996 & 14162 & 16265 \\
\hline B & $\mathrm{zW}$ & 127 & $\begin{array}{l}155 \\
\end{array}$ & 208 & $\begin{array}{l}219 \\
548\end{array}$ & 233 & 314 & 343 & 379 & 445 & 401 & 413 \\
\hline $\begin{array}{l}\text { Cypru } \\
\text { Czech }\end{array}$ & $\begin{array}{l}\text { A7RT } \\
\text { LGIN }\end{array}$ & $\begin{array}{r}468 \\
1094\end{array}$ & $\begin{array}{r}424 \\
1259\end{array}$ & $\begin{array}{r}490 \\
1215\end{array}$ & $\begin{array}{r}548 \\
1250\end{array}$ & $\begin{array}{r}656 \\
1306\end{array}$ & $\begin{array}{r}669 \\
1414\end{array}$ & $\begin{array}{l}1407 \\
1926\end{array}$ & $\begin{array}{r}944 \\
1923\end{array}$ & $\begin{array}{l}1141 \\
1994\end{array}$ & $\begin{array}{l}1171 \\
1907\end{array}$ & $\begin{array}{l}1164 \\
2279\end{array}$ \\
\hline Denm & $\begin{array}{l}\mathrm{LGIN} \\
\mathrm{LGHV}\end{array}$ & $\begin{array}{l}1094 \\
3496\end{array}$ & $\begin{array}{l}1259 \\
3659 \\
3\end{array}$ & $\begin{array}{l}1215 \\
4104\end{array}$ & $\begin{array}{l}1250 \\
3702\end{array}$ & $\begin{array}{l}1306 \\
3636\end{array}$ & $\begin{array}{l}1414 \\
3847\end{array}$ & $\begin{array}{l}\begin{array}{l}1926 \\
6185\end{array} \\
6\end{array}$ & $\begin{array}{l}1923 \\
4581\end{array}$ & $\begin{array}{l}1994 \\
5014\end{array}$ & $\begin{array}{l}1907 \\
5364\end{array}$ & 5667 \\
\hline Esto & zWLX & 108 & 97 & 121 & 115 & 135 & 152 & 528 & 264 & 270 & 183 & 272 \\
\hline $\begin{array}{l}\text { Finla } \\
\text { Frand }\end{array}$ & LGHW & $\begin{array}{l}2425 \\
24015\end{array}$ & $\begin{array}{r}2493 \\
2507\end{array}$ & $\begin{array}{r}2273 \\
24778\end{array}$ & $\begin{array}{r}2368 \\
25120\end{array}$ & $\begin{array}{r}2321 \\
0\end{array}$ & $\begin{array}{r}2376 \\
06\end{array}$ & 2902 & $\begin{array}{l}3183 \\
3\end{array}$ & $\begin{array}{r}3104 \\
3\end{array}$ & $\begin{array}{r}2627 \\
05055\end{array}$ & $\begin{array}{r}2772 \\
2814\end{array}$ \\
\hline $\begin{array}{l}\text { France } \\
\text { Germany }\end{array}$ & $\begin{array}{l}\text { LGHX } \\
\text { LGHY }\end{array}$ & $\begin{array}{l}24015 \\
29465\end{array}$ & $\begin{array}{l}\begin{array}{l}53327 \\
30849\end{array} \\
\text {. }\end{array}$ & $\begin{array}{l}\begin{array}{l}24778 \\
29562\end{array} \\
2952\end{array}$ & $\begin{array}{l}\begin{array}{l}25122 \\
28637\end{array} \\
28637\end{array}$ & $\begin{array}{l}25175 \\
29885\end{array}$ & $\begin{array}{l}\begin{array}{l}626390 \\
32197\end{array}\end{array}$ & $\begin{array}{l}\begin{array}{l}36388 \\
37261\end{array}\end{array}$ & $\begin{array}{l}26534 \\
35513\end{array}$ & $\begin{array}{l}28018 \\
39816\end{array}$ & $\begin{array}{l}\begin{array}{l}25955 \\
35746\end{array}\end{array}$ & $\begin{array}{l}\begin{array}{l}88141 \\
38713\end{array} \\
38713\end{array}$ \\
\hline $\begin{array}{l}\text { Germany } \\
\text { Greece }\end{array}$ & $\begin{array}{l}\text { LGHY } \\
\text { LGHZ }\end{array}$ & $\begin{array}{r}29465 \\
2066\end{array}$ & $\begin{array}{r}r 0849 \\
1920\end{array}$ & $\begin{array}{r}29562 \\
1965\end{array}$ & $\begin{array}{r}88637 \\
2037\end{array}$ & $\begin{array}{r}29885 \\
2149\end{array}$ & $\begin{array}{r}r 2197 \\
2304\end{array}$ & $\begin{array}{r}77261 \\
2257\end{array}$ & $\begin{array}{r}35513 \\
2295\end{array}$ & $\begin{array}{r}39816 \\
2918\end{array}$ & $\begin{array}{r}35746 \\
2658\end{array}$ & $\begin{array}{r}88713 \\
2500\end{array}$ \\
\hline Greece & $\begin{array}{l}\text { LGHZ } \\
\text { XUXI }\end{array}$ & $\begin{array}{r}2066 \\
823\end{array}$ & $\begin{array}{r}1920 \\
821\end{array}$ & $\begin{array}{r}1965 \\
968\end{array}$ & $\begin{array}{l}203 / \\
1079\end{array}$ & $\begin{array}{l}2149 \\
1310\end{array}$ & $\begin{array}{l}2304 \\
1394\end{array}$ & $\begin{array}{l}225 / \\
1265\end{array}$ & $\begin{array}{l}2295 \\
1373\end{array}$ & $\begin{array}{l}2918 \\
1491\end{array}$ & $\begin{array}{l}2658 \\
1402\end{array}$ & $\begin{array}{l}2500 \\
1554\end{array}$ \\
\hline Ireland & LGIA & 15222 & 17496 & 19708 & 17521 & $\begin{array}{l}19723 \\
1972\end{array}$ & 22864 & 24059 & 25418 & 27769 & 24368 & $\begin{array}{r}1504 \\
25321\end{array}$ \\
\hline it & B & $\begin{array}{l}15222 \\
11 \\
1220\end{array}$ & $\begin{array}{l}11307 \\
11\end{array}$ & 11527 & $\begin{array}{l}11621 \\
11623\end{array}$ & 11814 & $\begin{array}{l}22804 \\
12501\end{array}$ & $\begin{array}{l}13233 \\
1323\end{array}$ & $\begin{array}{l}5461 \\
3461\end{array}$ & $\begin{array}{l}13935 \\
13909\end{array}$ & 13344 & $\begin{array}{l}14004 \\
14004\end{array}$ \\
\hline $\mathrm{L}$ & & 114 & 117 & 114 & 141 & 40 & 94 & 654 & 249 & 258 & 219 & 255 \\
\hline Lithuan & ZWME & 148 & 182 & 181 & 31 & 197 & 279 & 340 & 432 & 560 & 265 & 321 \\
\hline Luxe & A7RV & 623 & 736 & 805 & 952 & 1167 & 1069 & 2763 & 2027 & 2370 & 2556 & 2390 \\
\hline $\begin{array}{l}\text { Malta } \\
\text { Nethe }\end{array}$ & $\begin{array}{l}\text { A7 RW } \\
\text {. }\end{array}$ & $\begin{array}{r}269 \\
19724\end{array}$ & $\begin{array}{r}267 \\
19517\end{array}$ & $\begin{array}{r}286 \\
18721\end{array}$ & & & $\begin{array}{r}310 \\
19645\end{array}$ & $\begin{array}{r}411 \\
24425\end{array}$ & $\begin{array}{r}485 \\
23717\end{array}$ & & $\begin{array}{r}684 \\
2721\end{array}$ & $\begin{array}{r}620 \\
31319\end{array}$ \\
\hline rlands & $\begin{array}{l}\text { LGIC } \\
\text { LGIO }\end{array}$ & $\begin{array}{r}19724 \\
1585\end{array}$ & $\begin{array}{r}19517 \\
1634\end{array}$ & $\begin{array}{r}88721 \\
1707\end{array}$ & $\begin{array}{r}8988 \\
1870\end{array}$ & $\begin{array}{r}18724 \\
1927\end{array}$ & $\begin{array}{r}19645 \\
2455\end{array}$ & $\begin{array}{r}24425 \\
3809\end{array}$ & $\begin{array}{r}23 \\
3391\end{array}$ & $\begin{array}{r}29847 \\
4092\end{array}$ & $\begin{array}{r}7431 \\
3851\end{array}$ & $\begin{array}{r}1319 \\
4936\end{array}$ \\
\hline Portu & $\begin{array}{l}L G I 0 \\
\text { LGID }\end{array}$ & $\begin{array}{l}1585 \\
2099\end{array}$ & $\begin{array}{l}1034 \\
2060\end{array}$ & 2046 & $\begin{array}{l}1996 \\
\end{array}$ & 2259 & $\begin{array}{l}2453 \\
2330\end{array}$ & $\begin{array}{l}3809 \\
2913\end{array}$ & $\begin{array}{l}3391 \\
2164\end{array}$ & $\begin{array}{l}4092 \\
2453\end{array}$ & 2314 & $\begin{array}{l}4936 \\
2616\end{array}$ \\
\hline Rom & zWMH & 423 & 412 & 503 & 604 & 745 & 773 & 771 & 927 & 1037 & 969 & 1140 \\
\hline epublic & ZWMJ & $\begin{array}{l}195 \\
181\end{array}$ & $\begin{array}{l}244 \\
195\end{array}$ & $\begin{array}{l}230 \\
229\end{array}$ & 270 & $\begin{array}{l}269 \\
212\end{array}$ & 358 & $\begin{array}{r}389 \\
269\end{array}$ & 525 & $\begin{array}{l}761 \\
316\end{array}$ & 620 & $\begin{array}{l}757 \\
312\end{array}$ \\
\hline $\begin{array}{l}\text { Solven } \\
\text { Spain }\end{array}$ & ZWMI & $\begin{array}{r}187 \\
10557\end{array}$ & $\begin{array}{r}195 \\
10617\end{array}$ & $\begin{array}{r}229 \\
11102\end{array}$ & $\begin{array}{r}202 \\
11812\end{array}$ & 12047 & $\begin{array}{r}231 \\
14436\end{array}$ & $\begin{array}{r}269 \\
17068\end{array}$ & $\begin{array}{r}216 \\
15319\end{array}$ & 15741 & $\begin{array}{r}259 \\
14905\end{array}$ & $\begin{array}{r}312 \\
15079\end{array}$ \\
\hline $\begin{array}{l}\text { Spa } \\
\text { Swe }\end{array}$ & $\begin{array}{l}\text { LGIE } \\
\text { LGIF }\end{array}$ & 5833 & 5590 & 5350 & 5520 & 6065 & 6349 & 7183 & 7299 & $\begin{array}{l}15141 \\
7594\end{array}$ & 6840 & 8474 \\
\hline $\begin{array}{l}\text { European Central Bank } \\
\text { EU Institutions }\end{array}$ & $\begin{array}{l}\text { ZWLI } \\
\text { LGIG }\end{array}$ & $\begin{array}{r}3 \\
248\end{array}$ & $\begin{array}{r}12 \\
544\end{array}$ & $\begin{array}{r}3 \\
544\end{array}$ & 513 & 578 & 602 & & 627 & & & \\
\hline Total EU27 & GC 89 & 146156 & 151223 & 152995 & 152962 & 157749 & 171303 & 207311 & 190174 & 211516 & 192908 & 210148 \\
\hline European Free $\mathrm{Tr}$ & & & & & & & & & & & & \\
\hline $\begin{array}{l}\text { Icel } \\
\text { Lied }\end{array}$ & LGII & 266 & 214 & 197 & 216 & 259 & $\begin{array}{r}313 \\
42\end{array}$ & 378 & 381 & $\begin{array}{r}419 \\
68\end{array}$ & 275 & 300 \\
\hline $\begin{array}{l}\text { lech } \\
\text { Norw }\end{array}$ & $\begin{array}{l}\text { LGIJ } \\
\text { LGIK }\end{array}$ & $\begin{array}{r}44 \\
3103\end{array}$ & 2856 & $\begin{array}{r}46 \\
3239\end{array}$ & $\begin{array}{l}45 \\
3272\end{array}$ & $\begin{array}{r}59 \\
3528\end{array}$ & $\begin{array}{r}42 \\
4075\end{array}$ & $\begin{array}{r}65 \\
4119\end{array}$ & $\begin{array}{r}130 \\
5272\end{array}$ & 5309 & 5263 & $\begin{array}{r}85 \\
5497\end{array}$ \\
\hline & & & 7103 & 7227 & 7498 & 7292 & 9955 & 9904 & 10570 & 11941 & 11874 & 14195 \\
\hline Total EFTA & LGIM & 9337 & 10208 & 10709 & 11031 & 11138 & 14385 & 14467 & 16353 & 17737 & 17489 & 20077 \\
\hline Other Europ & & & & & & & & & & & & \\
\hline $\begin{array}{l}\text { Alb } \\
\text { Be }\end{array}$ & $\mathrm{zWL}$ & $\begin{array}{r}9 \\
63\end{array}$ & 28 & 25 & 17 & 34 & 24 & 30 & 30 & 46 & 31 & $\begin{array}{r}33 \\
130\end{array}$ \\
\hline & $\begin{array}{l}\mathrm{ZWI} \\
\mathrm{ZWM}\end{array}$ & 111 & $\begin{array}{r}36 \\
121\end{array}$ & $\begin{array}{r}36 \\
149\end{array}$ & $\begin{array}{c}42 \\
188\end{array}$ & $\begin{array}{r}59 \\
168\end{array}$ & $\begin{array}{r}63 \\
162\end{array}$ & 243 & $\begin{array}{r}84 \\
257\end{array}$ & $\begin{array}{l}109 \\
338\end{array}$ & $\begin{array}{r}85 \\
328\end{array}$ & 255 \\
\hline & LGIP & 1087 & 1367 & 1571 & 2143 & 2374 & 2986 & 3727 & 4929 & 6452 & 4233 & 5260 \\
\hline & LGIQ & 2193 & 1589 & 1729 & 2097 & 2331 & 2711 & 3033 & 3110 & 3333 & 3293 & 4374 \\
\hline Ukr & ZWMK & 195 & 224 & 473 & 364 & 269 & 373 & 487 & 647 & 840 & 766 & 718 \\
\hline Serbia and Monte & ZWMN & 135 & 92 & 90 & 105 & 87 & 58 & 131 & 170 & & 173 & 156 \\
\hline of which & KN & & & & & & & & 156 & 155 & $\begin{array}{l}155 \\
18\end{array}$ & 141 \\
\hline Other & $\begin{array}{l}\text { KNA2 } \\
\text { ZWLM }\end{array}$ & 1438 & 1385 & $169 \ddot{3}$ & 2249 & $246 \ddot{2}$ & 3043 & 3486 & $\begin{array}{l}144 \\
3936\end{array}$ & $\begin{array}{r}13 \\
5441\end{array}$ & $\begin{array}{r}18 \\
4611\end{array}$ & $\begin{array}{r}15 \\
4132\end{array}$ \\
\hline Total Europe & LGIS & 160724 & 166273 & 169470 & 171198 & 176671 & 195108 & 232990 & 219690 & 245980 & 223917 & 245283 \\
\hline nerica & & & & & & & & & & & & \\
\hline tina & zWI & 476 & 457 & 204 & 212 & 313 & 258 & 325 & 356 & 457 & 401 & $\begin{array}{r}590 \\
3053\end{array}$ \\
\hline & & $\begin{array}{l}1122 \\
4862\end{array}$ & $\begin{array}{l}1202 \\
4831\end{array}$ & $\begin{array}{l}1202 \\
4630\end{array}$ & $\begin{array}{l}1155 \\
4809\end{array}$ & $\begin{array}{l}1108 \\
5090\end{array}$ & $\begin{array}{l}1214 \\
5009\end{array}$ & $\begin{array}{l}1409 \\
5901\end{array}$ & $\begin{array}{l}1521 \\
5\end{array}$ & 2279 & 2482 & $\begin{array}{l}3053 \\
7032\end{array}$ \\
\hline $\mathrm{C}$ & & $\begin{array}{r}4862 \\
209\end{array}$ & $\begin{array}{r}4830 \\
230\end{array}$ & $\begin{array}{r}4630 \\
200\end{array}$ & $\begin{aligned} 4809 \\
200\end{aligned}$ & 5090 & 5009 & $\begin{aligned} 5 \\
316\end{aligned}$ & $5 / 86$ & 5439 & 6029 & 811 \\
\hline $\mathrm{C}$ & & 156 & 244 & 167 & 203 & 2 & 179 & 207 & 213 & & 265 & $\begin{array}{l}811 \\
430\end{array}$ \\
\hline $\mathrm{M}$ & & 942 & 971 & & 53 & 77 & 937 & 1136 & 1119 & 1230 & 1135 & 1373 \\
\hline tes of America & LGIW & 48646 & 48614 & 50832 & 52705 & 54293 & 55337 & 61499 & 65582 & 70765 & 67686 & 72211 \\
\hline Urugua & $\mathrm{zW}$ & 64 & 53 & 35 & 93 & 41 & 50 & 59 & 53 & 33 & 75 & 99 \\
\hline jean Countries & & 300 & 423 & 402 & 241 & 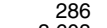 & 322 & 306 & & & 375 & 627 \\
\hline tral American Countries & $\mathrm{zW}$ & $\begin{array}{l}2388 \\
380\end{array}$ & $\begin{array}{l}2285 \\
383\end{array}$ & 2892 & $\begin{array}{l}3076 \\
330\end{array}$ & $\begin{array}{r}3608 \\
300\end{array}$ & $\begin{array}{l}4172 \\
351\end{array}$ & $\begin{array}{r}4437 \\
396\end{array}$ & $\begin{array}{l}5050 \\
492\end{array}$ & 5881 & 5212 & 5108 \\
\hline $\begin{array}{l}\text { Total Americas } \\
\text { Torice }\end{array}$ & $\begin{array}{l}\text { ZWLIY } \\
\text { LGI }\end{array}$ & $\begin{array}{l}380 \\
59545\end{array}$ & $\begin{array}{l}383 \\
59693\end{array}$ & 61837 & 63977 & 66435 & 68066 & $\begin{array}{l}35961 \\
75991\end{array}$ & 80841 & 88190 & 84862 & 92005 \\
\hline Asia & & & & & & & & & & & & \\
\hline Chin & $L G=$ & 1910 & 2297 & 220 & 2794 & 364 & 418 & 47 & 5324 & 7572 & 7631 & $\begin{array}{r}10263 \\
6062\end{array}$ \\
\hline & & & $\begin{array}{l}3770 \\
2\end{array}$ & 34 & 3663 & & $\begin{array}{l}4430 \\
3\end{array}$ & 41 & & & 5448 & \\
\hline In & & $\begin{array}{r}2599 \\
606\end{array}$ & $\begin{array}{l}2438 \\
515\end{array}$ & 23 & 2988 & $\begin{array}{r}3233 \\
593\end{array}$ & $\begin{array}{r}3954 \\
567\end{array}$ & $\begin{array}{l}4346 \\
609\end{array}$ & 4665 & & $\begin{array}{r}4660 \\
692\end{array}$ & $\begin{array}{c}6128 \\
786\end{array}$ \\
\hline In & & 424 & 606 & 58 & $\begin{array}{l}0<4 \\
702\end{array}$ & & 676 & & 5 & & $\begin{array}{l}692 \\
618\end{array}$ & $\begin{array}{l}100 \\
435\end{array}$ \\
\hline Is & ${ }_{\mathrm{LG}}$ & $\begin{array}{l}4957 \\
1957\end{array}$ & 1867 & $\begin{array}{r}1800 \\
1849\end{array}$ & 1729 & 179 & 1903 & $\begin{array}{l}1763 \\
1763\end{array}$ & 1723 & $1 \varepsilon$ & $\begin{array}{l}1730 \\
1730\end{array}$ & 2069 \\
\hline $\mathrm{Ja}$ & & 7126 & 7226 & 7431 & 7591 & 80 & 8212 & 84 & 85 & & 8207 & 8649 \\
\hline IVI & $\bar{L} C$ & 1293 & 1403 & 1278 & 1444 & 15 & 1546 & 13 & 1402 & 1661 & 1726 & 1973 \\
\hline$P$ & & 374 & 494 & 4 & 466 & & 893 & & 7 & & 835 & 63 \\
\hline$S$ & & 452 & $\begin{array}{r}572 \\
3651\end{array}$ & $\begin{array}{r}462 \\
3\end{array}$ & $\begin{array}{r}520 \\
4135\end{array}$ & & $\begin{array}{l}412 \\
3808\end{array}$ & $\begin{array}{r}340 \\
412\end{array}$ & 359 & & $\begin{array}{r}445 \\
7773\end{array}$ & $\begin{array}{r}409 \\
5\end{array}$ \\
\hline $\begin{array}{l}\text { Sa } \\
\text { Si }\end{array}$ & $\mathrm{LG}$ & 3 & $\begin{array}{l}3651 \\
2882\end{array}$ & $\begin{array}{r}3349 \\
2605\end{array}$ & $\begin{array}{l}4135 \\
3219\end{array}$ & & 8 & $\begin{array}{l}4212 \\
5331\end{array}$ & 40 & & $\begin{array}{l}4773 \\
6959\end{array}$ & $\begin{array}{l}5413 \\
7108\end{array}$ \\
\hline 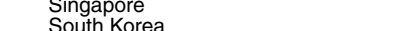 & & $\begin{array}{l}2704 \\
1742\end{array}$ & $\begin{array}{l}2882 \\
1687\end{array}$ & $\begin{array}{l}26 \\
19\end{array}$ & $\begin{array}{r}3219 \\
1948\end{array}$ & & $\begin{array}{l}4719 \\
2413\end{array}$ & $\begin{array}{l}5331 \\
2609\end{array}$ & 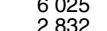 & & $\begin{array}{l}6959 \\
3088\end{array}$ & $\begin{array}{l}7108 \\
3467\end{array}$ \\
\hline sc & & $\begin{array}{l}1 / 42 \\
1291\end{array}$ & $\begin{array}{l}188 \\
1169\end{array}$ & $\begin{array}{l}1984 \\
1212\end{array}$ & $\begin{array}{l}1948 \\
1273\end{array}$ & 1466 & $\begin{array}{l}2413 \\
1523\end{array}$ & 1503 & 1578 & $\begin{array}{l}3604 \\
1574\end{array}$ & 1456 & $\begin{array}{l}346 / \\
2261\end{array}$ \\
\hline 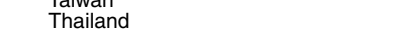 & & 773 & 815 & 737 & 903 & $\begin{array}{l}400 \\
955\end{array}$ & 891 & 860 & $\begin{array}{l}15 \\
9\end{array}$ & & 1411 & $\begin{array}{l}1501 \\
1596\end{array}$ \\
\hline $\mathrm{R}$ & & 3715 & 3817 & 3920 & 4702 & 5782 & 9042 & 7473 & 7350 & 9 & & 10597 \\
\hline Middle East & & 619 & 803 & 748 & & 1. & & 12 & & & 1743 & 1926 \\
\hline S & & & & & 1 & & & $2 \pi(8+2)+2$ & & & 8 & 38 \\
\hline Total Asia & LGJO & 36695 & 37447 & 36366 & 40966 & 45606 & 52026 & 52868 & 55116 & 65264 & 63750 & 72843 \\
\hline Austr & & & & & & & & & & & & \\
\hline A & LG. & 4281 & 4262 & 394 & 4308 & 4743 & 5292 & 5338 & 5889 & 7160 & 7139 & 8387 \\
\hline Net & & & 605 & & 6 & & & & 7 & & 776 & 866 \\
\hline & & ק2 & 141 & 154 & 185 & 571 & 143 & 198 & 17 & & & \\
\hline Total Australasia \& Oceania & LGJS & 5023 & 5008 & 4687 & 5143 & 5717 & 6263 & 6317 & 6858 & 8217 & 8139 & 9543 \\
\hline ica & & & & & & & & & & & & \\
\hline & $\mathrm{zw}-1$ & 78 & 79 & 686 & 697 & 991 & 917 & 10 & 1320 & 1668 & 1663 & 1758 \\
\hline & & & 2654 & & & & & & & & & \\
\hline & & 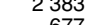 & 2654 & 25 & & & & & & & & 4613 \\
\hline & & & 645 & 6 & & & & & & & & \\
\hline 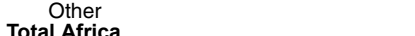 & IG & 3367 & $\begin{array}{l}3749 \\
8290\end{array}$ & $\begin{array}{l}3672 \\
8009\end{array}$ & $\begin{array}{l}4055 \\
8850\end{array}$ & $\begin{array}{l}3974 \\
9131\end{array}$ & $\begin{array}{l}4275 \\
9544\end{array}$ & $\begin{array}{r}4986 \\
10883\end{array}$ & $\begin{array}{r}5251 \\
11480\end{array}$ & $\begin{array}{r}7312 \\
15163\end{array}$ & 7457 & $\begin{array}{r}8597 \\
17081\end{array}$ \\
\hline International Organisations & LGJW & 51 & 64 & 85 & 73 & 52 & 60 & 42 & 47 & 50 & 39 & 41 \\
\hline rld total & KTMW & 9714 & 276775 & 280454 & 0207 & 303612 & 1067 & 379091 & 374032 & 422864 & 55588 & 36796 \\
\hline
\end{tabular}

1 Before 1999 only combined BLEU data is available under indentifier LGHU. 2 Separate data for Serbia and Montenegro available from 2007. 
9.3 Trade in goods and services

\begin{tabular}{|c|c|c|c|c|c|c|c|c|c|c|c|c|}
\hline continued & & & & & & & & & & & & $£$ million \\
\hline & & 2000 & 2001 & 2002 & 2003 & 2004 & 2005 & 2006 & 2007 & 2008 & 2009 & 2010 \\
\hline Imports & & & & & & & & & & & & \\
\hline $\begin{array}{l}\text { Europe } \\
\text { European Unior }\end{array}$ & & & & & & & & & & & & \\
\hline $\begin{array}{l}\text { European Unior } \\
\text { Austria }\end{array}$ & LGJY & 1894 & 2391 & 3083 & 3436 & 3112 & 3340 & 3667 & 3311 & 3318 & 3188 & 3620 \\
\hline $\begin{array}{l}\text { Belgium }{ }^{1} \\
\text { Bulgaria }\end{array}$ & A8EO & 11978 & 132 & 14164 & 14302 & 14790 & 16266 & 17416 & 17332 & 19009 & 17411 & 19400 \\
\hline $\begin{array}{l}\text { Bulgaria } \\
\text { Cyprus }\end{array}$ & $\begin{array}{l}\text { ZWNX } \\
\text { A8EP }\end{array}$ & $\begin{array}{r}125 \\
1090\end{array}$ & $\begin{array}{r}152 \\
1297\end{array}$ & $\begin{array}{r}213 \\
1223\end{array}$ & $\begin{array}{r}230 \\
1342\end{array}$ & $\begin{array}{r}324 \\
1289\end{array}$ & $\begin{array}{r}427 \\
1479\end{array}$ & $\begin{array}{r}438 \\
2896\end{array}$ & $\begin{array}{r}572 \\
1328\end{array}$ & $\begin{array}{r}609 \\
1322\end{array}$ & $\begin{array}{r}448 \\
1057\end{array}$ & $\begin{array}{l}571 \\
987\end{array}$ \\
\hline Czech Republic & $\begin{array}{l}\text { AOEF } \\
\text { LGKS }\end{array}$ & 969 & 1298 & $\begin{array}{l}1500 \\
1500\end{array}$ & $\begin{array}{l}1753 \\
1753\end{array}$ & 1716 & 2348 & $\begin{array}{l}3488 \\
3488 \\
3\end{array}$ & $\begin{array}{l}3467 \\
3467\end{array}$ & 4066 & 3708 & 4422 \\
\hline Den & LGKA & 3313 & 3582 & 4350 & 4185 & 4220 & 5358 & 7557 & 4492 & 5063 & 5266 & 5411 \\
\hline Estonia & $\mathrm{zWC}$ & 345 & 307 & 340 & 299 & 427 & 418 & 2188 & 293 & 206 & 157 & 196 \\
\hline $\begin{array}{l}\text { Finland } \\
\text { France }\end{array}$ & $\begin{array}{l}\text { LGKB } \\
\text { LGKC }\end{array}$ & $\begin{array}{r}3168 \\
26207\end{array}$ & $\begin{array}{r}3323 \\
27665\end{array}$ & $\begin{array}{r}3071 \\
28651\end{array}$ & $\begin{array}{r}2977 \\
28655\end{array}$ & $\begin{array}{r}2625 \\
28668\end{array}$ & $\begin{array}{r}2785 \\
31000\end{array}$ & $\begin{array}{r}3431 \\
36335\end{array}$ & $\begin{array}{r}3040 \\
31827\end{array}$ & $\begin{array}{r}3201 \\
33206\end{array}$ & $\begin{array}{r}2467 \\
29723\end{array}$ & $\begin{array}{r}2615 \\
31108\end{array}$ \\
\hline $\begin{array}{l}\text { France } \\
\text { Germany }\end{array}$ & $\begin{array}{l}\text { LGKC } \\
\text { LGKD }\end{array}$ & $\begin{array}{l}26200 \\
33208\end{array}$ & $\begin{array}{l}27665 \\
35447\end{array}$ & $\begin{array}{l}28651 \\
37852\end{array}$ & $\begin{array}{l}28655 \\
39257\end{array}$ & $\begin{array}{l}28668 \\
41849\end{array}$ & $\begin{array}{l}31000 \\
46903\end{array}$ & $\begin{array}{l}36335 \\
49854\end{array}$ & $\begin{array}{l}31822 \\
53022\end{array}$ & $\begin{array}{l}33206 \\
54600\end{array}$ & $\begin{array}{l}48918 \\
4893\end{array}$ & $\begin{array}{l}31208 \\
55542\end{array}$ \\
\hline Gre & LGKE & $\begin{array}{r}2069 \\
707\end{array}$ & 2372 & 2503 & $\begin{array}{r}2625 \\
1620\end{array}$ & 2635 & 2719 & 2821 & 2888 & 2809 & 2670 & 2652 \\
\hline $\begin{array}{l}\text { Hungary } \\
\text { Hreland }\end{array}$ & zWOJ & 775 & $\begin{array}{r}833 \\
14528\end{array}$ & $\begin{array}{r}975 \\
15\end{array}$ & $\begin{array}{r}1269 \\
12907\end{array}$ & $\begin{array}{r}1767 \\
13195\end{array}$ & $\begin{array}{l}2136 \\
13819\end{array}$ & $\begin{array}{r}2699 \\
11212\end{array}$ & $\begin{array}{l}2746 \\
15312\end{array}$ & $\begin{array}{r}2880 \\
1644\end{array}$ & 2958 & 3737 \\
\hline $\begin{array}{l}\text { Ireland } \\
\text { Italy }\end{array}$ & $\begin{array}{l}\text { LGKF } \\
\text { LGKG }\end{array}$ & $\begin{array}{l}12548 \\
12383\end{array}$ & $\begin{array}{l}14528 \\
12857\end{array}$ & $\begin{array}{l}15535 \\
13812\end{array}$ & $\begin{array}{l}12497 \\
14910\end{array}$ & $\begin{array}{l}13195 \\
15763\end{array}$ & $\begin{array}{l}13849 \\
16994\end{array}$ & $\begin{array}{l}14212 \\
17041\end{array}$ & $\begin{array}{l}15332 \\
18030\end{array}$ & $\begin{array}{l}16464 \\
19115\end{array}$ & $\begin{array}{l}16685 \\
16773\end{array}$ & $\begin{array}{ll}17023 \\
18290\end{array}$ \\
\hline Latvic & $\begin{array}{l}\text { LGKG } \\
\text { ZWOM }\end{array}$ & $\begin{array}{l}12383 \\
432\end{array}$ & $\begin{array}{r}12857 \\
450\end{array}$ & $\begin{array}{l}13812 \\
495\end{array}$ & $\begin{array}{r}14910 \\
546\end{array}$ & $\begin{array}{l}15763 \\
728\end{array}$ & $\begin{array}{l}16994 \\
762\end{array}$ & $\begin{array}{r}17041 \\
880\end{array}$ & $\begin{array}{l}18030 \\
718\end{array}$ & $\begin{array}{r}19115 \\
488\end{array}$ & $\begin{array}{l}167 / 33 \\
395\end{array}$ & $\begin{array}{r}18290 \\
534\end{array}$ \\
\hline Lithuani & zWOL & 250 & 245 & 300 & 312 & 289 & 351 & 367 & 405 & 446 & 471 & 669 \\
\hline Luxembourg ${ }^{1}$ & A8ER & $\begin{array}{l}407 \\
351\end{array}$ & 605 & 965 & 978 & 1289 & 1220 & $\begin{array}{r}3253 \\
\quad 61\end{array}$ & 1191 & 1434 & 1157 & 2348 \\
\hline $\begin{array}{l}\text { Malta } \\
\text { Netherlands }\end{array}$ & $\begin{array}{l}\text { A8ES } \\
\text { LGKH }\end{array}$ & $\begin{array}{r}331 \\
18424\end{array}$ & $\begin{array}{r}401 \\
18522\end{array}$ & $\begin{array}{r}408 \\
19529\end{array}$ & $\begin{array}{r}465 \\
20020\end{array}$ & $\begin{array}{r}502 \\
21566\end{array}$ & $\begin{array}{r}522 \\
24041\end{array}$ & $\begin{array}{l}461 \\
26059\end{array}$ & $\begin{array}{l}483 \\
26729\end{array}$ & $\begin{array}{r}517 \\
29697\end{array}$ & $\begin{array}{r}433 \\
26169\end{array}$ & $\begin{array}{r}496 \\
30347\end{array}$ \\
\hline $\begin{array}{l}\text { Netherlands } \\
\text { Poland }\end{array}$ & $\begin{array}{l}\text { LGKH } \\
\text { LGKT }\end{array}$ & $\begin{array}{r}18424 \\
1095\end{array}$ & $\begin{array}{r}8522 \\
1367\end{array}$ & $\begin{array}{r}19529 \\
1453\end{array}$ & $\begin{array}{r}20020 \\
1770\end{array}$ & $\begin{array}{r}21566 \\
2133\end{array}$ & $\begin{array}{r}r 4041 \\
2880\end{array}$ & $\begin{array}{r}26059 \\
4623\end{array}$ & $\begin{array}{r}26 \\
4914\end{array}$ & $\begin{array}{l}29697 \\
5624\end{array}$ & $\begin{array}{r}26169 \\
5924\end{array}$ & $\begin{array}{r}30347 \\
7467\end{array}$ \\
\hline Portus & LG! & 2755 & $\begin{array}{l}2740 \\
2740\end{array}$ & $\begin{array}{l}1453 \\
2970\end{array}$ & 3334 & $\begin{array}{l}2733 \\
3325\end{array}$ & $\begin{array}{l}2880 \\
3463\end{array}$ & $\begin{array}{l}4623 \\
4603\end{array}$ & $\begin{array}{l}4914 \\
3211\end{array}$ & $\begin{array}{l}5624 \\
3666\end{array}$ & $\begin{array}{l}5924 \\
2938\end{array}$ & 3382 \\
\hline & & 400 & & 603 & 772 & $\begin{array}{l}3325 \\
918\end{array}$ & $\begin{array}{r}3463 \\
971\end{array}$ & & 1119 & 1025 & $\begin{array}{l}2938 \\
1024\end{array}$ & $\begin{array}{l}3489 \\
1499\end{array}$ \\
\hline Slovak & & 168 & 188 & 238 & 282 & 296 & 439 & 939 & 1368 & 1752 & 1728 & 1741 \\
\hline Sloven & ZWOP & 152 & 166 & 196 & 195 & 205 & 252 & 803 & 361 & 386 & 291 & 405 \\
\hline Spai & LGKJ & 12647 & 14599 & 17045 & 18237 & 18236 & 21004 & 21952 & 20490 & 21383 & 19006 & 19863 \\
\hline Swe & & 5784 & 5501 & 5153 & 5703 & 6273 & 6783 & 7532 & 6529 & 8312 & 7484 & 8423 \\
\hline $\begin{array}{l}\text { European Cer } \\
\text { EU Institutions }\end{array}$ & $\underset{\mathrm{LGNR}}{\mathrm{ZWNR}}$ & & & & & & & & & & & \\
\hline Total EU27 & GCU & 152941 & 164624 & 176642 & 180368 & $188144^{4}$ & 208714 & 236514 & $22517 \overline{8}$ & 240605 & 218456 & 242850 \\
\hline European Free Trad & & & & & & & & & & & & \\
\hline $\begin{array}{l}\text { Icel } \\
\text { Lied }\end{array}$ & LGKN & $\begin{array}{c}450 \\
29\end{array}$ & 337 & 343 & 351 & 418 & $\begin{array}{r}421 \\
20\end{array}$ & 502 & 511 & 549 & $\begin{array}{r}619 \\
34\end{array}$ & 489 \\
\hline Norwa & $\begin{array}{l}\text { LGKO } \\
\text { LGKP }\end{array}$ & 6265 & 6410 & 6792 & 7345 & 9519 & 13413 & 15741 & 15637 & 23135 & 17411 & 22145 \\
\hline & & 7232 & 62 & 6455 & 5621 & 5326 & 6212 & 6710 & 7623 & 7996 & & 10644 \\
\hline Total EFTA & LGKR & 13976 & 13026 & 13617 & 13353 & 15285 & 20066 & 22998 & 23832 & 31722 & 26326 & 33308 \\
\hline Other Europ & & & & & & & & & & & & \\
\hline $\begin{array}{l}\text { Albania } \\
\text { Relarus }\end{array}$ & $\mathrm{zWNV}$ & 77 & 99 & $\begin{array}{l}15 \\
36\end{array}$ & 17 & $\begin{array}{l}20 \\
73\end{array}$ & 88 & 13 & $\begin{array}{r}22 \\
616\end{array}$ & $\begin{array}{r}20 \\
124\end{array}$ & 13 & \\
\hline $\begin{array}{l}\text { Belarus } \\
\text { Croatia }\end{array}$ & ${ }^{\mathrm{ZW}}$ & $\begin{array}{l}37 \\
69\end{array}$ & $\begin{array}{r}22 \\
108\end{array}$ & $\begin{array}{r}36 \\
117\end{array}$ & $\begin{array}{r}27 \\
120\end{array}$ & $\begin{array}{r}73 \\
138\end{array}$ & $\begin{array}{l}281 \\
193\end{array}$ & $\begin{array}{l}750 \\
243\end{array}$ & $\begin{array}{l}616 \\
228\end{array}$ & 124 & 26 & $\begin{array}{r}46 \\
318\end{array}$ \\
\hline Russ & LGKU & 1691 & $\begin{array}{r}108 \\
2282\end{array}$ & 2248 & 2762 & $\begin{array}{l}138 \\
4036\end{array}$ & 5611 & 6510 & 6303 & 8080 & 5935 & $\begin{array}{l}6161 \\
6161\end{array}$ \\
\hline Tu & $\mathrm{LG}$ & 1968 & 2291 & 2950 & 3 458 & 4127 & 4598 & 5062 & 5852 & 6461 & 5985 & 6974 \\
\hline Ukra & $\mathrm{zwC}$ & 74 & 93 & 163 & 105 & 143 & 174 & 195 & 313 & 376 & 297 & 500 \\
\hline Serbia and $M$ & zWOU & 45 & 61 & 63 & 75 & 76 & 61 & 138 & 145 & 150 & 137 & 143 \\
\hline $\begin{array}{r}\text { of which Ser } \\
\text { Mor }\end{array}$ & $\begin{array}{l}\text { KNA3 } \\
\text { KNA4 }\end{array}$ & & & & & & & & $\begin{array}{r}138 \\
7\end{array}$ & $\begin{array}{r}141 \\
9\end{array}$ & $\begin{array}{l}123 \\
14\end{array}$ & $\begin{array}{r}131 \\
12\end{array}$ \\
\hline Other & & 1208 & $110 \ddot{7}$ & $126 \ddot{0}$ & $121 \ddot{2}$ & 1107 & $122 \ddot{9}$ & $141 \ddot{2}$ & 1598 & 1482 & 1505 & 1522 \\
\hline Total Europe & LGKX & 172016 & 183623 & 197116 & 201497 & 213149 & 240935 & 273835 & 264087 & 289338 & 258955 & 291826 \\
\hline nericas & & & & & & & & & & & & \\
\hline & $\mathrm{zWI}$ & $\begin{array}{r}245 \\
\end{array}$ & 273 & 293 & 295 & 328 & 360 & 461 & 511 & 697 & 769 & $\begin{array}{r}777 \\
3558\end{array}$ \\
\hline $\mathrm{Br}$ & $\stackrel{L G}{L G}$ & $\begin{array}{l}1303 \\
5080\end{array}$ & $\begin{array}{l}1529 \\
4823\end{array}$ & $\begin{array}{l}1634 \\
4612\end{array}$ & $\begin{array}{l}1677 \\
4667\end{array}$ & $\begin{array}{l}1800 \\
5221\end{array}$ & $\begin{array}{l}2039 \\
5305\end{array}$ & $\begin{array}{l}2199 \\
6262\end{array}$ & $\begin{array}{l}2332 \\
7165\end{array}$ & $\begin{array}{l}3002 \\
7253\end{array}$ & $\begin{array}{l}2889 \\
5743\end{array}$ & $\begin{array}{l}3558 \\
7247\end{array}$ \\
\hline $\mathrm{Chi}$ & & $\begin{array}{r}5080 \\
510\end{array}$ & $\begin{array}{r}4823 \\
528\end{array}$ & 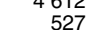 & $\begin{array}{r}4601 \\
471\end{array}$ & $\begin{array}{r}5221 \\
560\end{array}$ & $\begin{array}{r}5305 \\
573\end{array}$ & ${ }^{0} x_{5}+2$ & $\begin{array}{l}105 \\
565\end{array}$ & 635 & $\begin{array}{l}673 \\
673\end{array}$ & 634 \\
\hline $\mathrm{Co}$ & & 277 & $\begin{array}{l}5<0 \\
349\end{array}$ & $\begin{array}{l}327 \\
250\end{array}$ & 256 & 300 & 341 & 347 & $\begin{array}{l}505 \\
405\end{array}$ & 5 & 634 & $\begin{array}{l}733 \\
733\end{array}$ \\
\hline M & & 890 & 1067 & 827 & 789 & 764 & 730 & 701 & 914 & 1239 & 1022 & 1430 \\
\hline erica & LC & 41673 & 43364 & 38643 & 37871 & 37432 & 38350 & 42195 & 43677 & 45577 & 43445 & 46192 \\
\hline Uru & $\mathrm{zW}$ & 43 & 46 & 69 & 47 & 6 & 65 & 78 & 77 & 0 & 140 & 111 \\
\hline $\begin{array}{l}\text { Ver } \\
\text { Oth }\end{array}$ & $\mathrm{zW}$ & $\begin{array}{l}250 \\
2548\end{array}$ & $\begin{array}{l}197 \\
2031\end{array}$ & $\begin{array}{l}214 \\
2099\end{array}$ & $\begin{array}{r}146 \\
2435\end{array}$ & 3010 & $\begin{array}{r}425 \\
2985\end{array}$ & & 525 & & 470 & 471 \\
\hline an Cou & ZWC & $\begin{array}{r}2548 \\
544\end{array}$ & $\begin{array}{r}2031 \\
448\end{array}$ & $\begin{array}{r}2099 \\
392\end{array}$ & $\begin{array}{l}2435 \\
353\end{array}$ & $\begin{array}{r}3010 \\
347\end{array}$ & 2985 & 4083 & 3795 & $\begin{array}{r}4348 \\
525\end{array}$ & $\begin{array}{r}4376 \\
\quad 475\end{array}$ & $\begin{array}{l}3413 \\
487\end{array}$ \\
\hline $\begin{array}{l}\text { Other } \\
\text { Total Americas }\end{array}$ & $\begin{array}{l}\text { ZWOF } \\
\text { LGLD }\end{array}$ & $\begin{array}{r}5344 \\
53363\end{array}$ & $\begin{array}{r}448 \\
54655\end{array}$ & $\begin{array}{r}392 \\
49560\end{array}$ & $\begin{array}{r}353 \\
49007\end{array}$ & $\begin{array}{r}347 \\
50042\end{array}$ & $\begin{array}{r}395 \\
51568\end{array}$ & $\begin{array}{r}428 \\
57956\end{array}$ & $\begin{array}{rl} & 411 \\
60 & 377\end{array}$ & $\begin{array}{r}525 \\
64781\end{array}$ & $\begin{array}{r}475 \\
60636\end{array}$ & $\begin{array}{r}487 \\
65053\end{array}$ \\
\hline Asia & & & & & & & & & & & & \\
\hline $\mathrm{Ch}$ & $\mathrm{LG}$ & 5247 & 6280 & 7409 & 9006 & 11185 & 13936 & 16345 & 19885 & 24773 & 25804 & 31817 \\
\hline & $\mathrm{LG}$ & $\begin{array}{l}66 \\
23\end{array}$ & 64 & 62 & $\begin{array}{l}6303 \\
2887\end{array}$ & 65 & 7 & 8 & 81 & 9 & 8731 & $\begin{array}{l}9228 \\
8\end{array}$ \\
\hline & & 23 & & 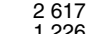 & & & & & & & & $\begin{array}{l}8104 \\
1507\end{array}$ \\
\hline & & $\begin{array}{l}1291 \\
64\end{array}$ & $\begin{array}{r}1323 \\
51\end{array}$ & 1 & $\begin{array}{l}1273 \\
60\end{array}$ & $\begin{array}{l}1084 \\
90\end{array}$ & $\begin{array}{l}1017 \\
67\end{array}$ & $\begin{array}{r}1166 \\
121\end{array}$ & & $\begin{array}{l}1345 \\
110\end{array}$ & $\begin{array}{l}1361 \\
285\end{array}$ & $\begin{array}{r}1507 \\
272\end{array}$ \\
\hline & & $\begin{array}{l}1276 \\
\end{array}$ & 12 & 1062 & 1052 & 1146 & 1245 & 1253 & 1348 & 1483 & 1448 & 1981 \\
\hline & & 11788 & 10906 & 9518 & 9826 & & 10866 & 10 & & & 9976 & 11734 \\
\hline$M$ & & 2574 & 2167 & 19 & 2067 & 2261 & 2100 & 2171 & 1934 & & & 2097 \\
\hline 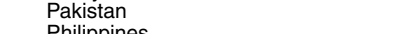 & & & & & 907 & 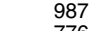 & & & & & 1091 & 1252 \\
\hline$\stackrel{P}{S}$ & & 1559 & $\begin{array}{l}1270 \\
1584\end{array}$ & 1068 & 881 & $\begin{array}{r}776 \\
\end{array}$ & & & & 3 & 577 & 742 \\
\hline $\begin{array}{c}S \\
\text { S }\end{array}$ & & & $\begin{array}{r}1584 \\
2482\end{array}$ & $\begin{array}{l}1990 \\
2404\end{array}$ & $\begin{array}{r}1793 \\
3124\end{array}$ & 1974 & & $\begin{array}{l}1970 \\
\end{array}$ & & & 905 & 1015 \\
\hline $\begin{array}{l}\text { Sin } \\
\text { Sol }\end{array}$ & LG & $\begin{array}{l}2769 \\
3523\end{array}$ & $\begin{array}{l}2482 \\
2930\end{array}$ & $\begin{array}{l}2404 \\
290 ?\end{array}$ & $\begin{array}{l}3124 \\
2739\end{array}$ & $\begin{array}{l}4092 \\
3315\end{array}$ & $\begin{array}{l}4607 \\
3289\end{array}$ & $\begin{array}{l}4653 \\
3310\end{array}$ & $\begin{array}{l}5026 \\
3311\end{array}$ & $\begin{array}{l}5444 \\
3913\end{array}$ & 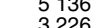 & $\begin{array}{l}\text { 5 } 949 \\
2914\end{array}$ \\
\hline Tai & $\begin{array}{l}\mathrm{LG} \\
\mathrm{LG}\end{array}$ & 3809 & 30 & 2628 & 2419 & 2587 & $\begin{array}{l}5289 \\
2572\end{array}$ & 2598 & 2727 & 2982 & $\begin{array}{l}2520 \\
2538\end{array}$ & 3365 \\
\hline & & 2025 & 21 & $\begin{array}{l}2020 \\
2108\end{array}$ & 2192 & 2439 & 2360 & 26 & 2711 & 3 & 2951 & $\begin{array}{l}3307 \\
330\end{array}$ \\
\hline $\mathrm{R}$ & & 15 & 16 & 18 & 23 & 26 & 30 & & 36 & & 4 & 7169 \\
\hline Other Near \& Middle Eastern Countries & $\mathrm{zW}$ & 2 & 2 & 3 & 307 & & 401 & 969 & & & 571 & 527 \\
\hline Total As $>2+a)$ & $\mathrm{zW}$ & 2208 & 2159 & 2250 & 2606 & 3109 & 2896 & 3492 & 3512 & 4 & 4623 & 5009 \\
\hline Total Asia & LGLT & 50916 & 49286 & 48501 & 51784 & 57739 & 64132 & 69707 & 73834 & 85903 & 82494 & 97989 \\
\hline Ononia & & & & & & & & & & & & \\
\hline A & LGI & 2650 & 2969 & 30 & 3217 & 3219 & 3 & 3 & 3 & 44 & 3964 & 4509 \\
\hline 0 & & & & & 4 & 1012 & 7 & 1043 & 8 & $?$ & 1102 & 1198 \\
\hline $\begin{array}{l}\text { Oth } \\
\text { Total Au }\end{array}$ & LGI & $\begin{array}{r}154 \\
3603\end{array}$ & $\begin{array}{r}119 \\
3917\end{array}$ & & $\begin{array}{r}154 \\
4265\end{array}$ & $\begin{array}{r}181 \\
4412\end{array}$ & $\begin{array}{r}179 \\
5194\end{array}$ & $\begin{array}{r}206 \\
5136\end{array}$ & $\begin{array}{r}164 \\
5218\end{array}$ & 5794 & $\begin{array}{r}223 \\
5289\end{array}$ & $\begin{array}{r}159 \\
5866\end{array}$ \\
\hline 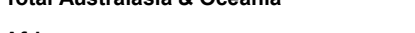 & & & & & & & & & & & & \\
\hline Africa & & & & & & & & & & & & \\
\hline & zWC & 700 & $\begin{array}{l}769 \\
592\end{array}$ & 722 & $\begin{array}{l}778 \\
587\end{array}$ & 835 & 874 & 1267 & 1126 & 1411 & 1428 & 1340 \\
\hline & & & $\begin{array}{r}5 \\
34\end{array}$ & $\begin{array}{r}5 \\
34 \\
\end{array}$ & $\begin{array}{r}587 \\
3912\end{array}$ & & 5 & & 4 & & $\begin{array}{r}5990 \\
4737\end{array}$ & $\begin{array}{r}591 \\
5524\end{array}$ \\
\hline & & 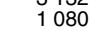 & 878 & 874 & 855 & 1093 & 1356 & 20 & 2105 & $2 \varepsilon$ & 2196 & 3283 \\
\hline & & 227 & 3472 & 36 & 3808 & 4054 & 4062 & 42 & 49 & 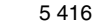 & 4885 & 4978 \\
\hline Africa & LGMA & 7753 & 9204 & 9210 & 9940 & 10876 & 11899 & 13131 & 13108 & 16129 & 13836 & 15716 \\
\hline nternational Organisations & LGMB & 80 & 62 & 40 & 29 & 37 & 43 & 57 & 57 & 43 & 15 & 30 \\
\hline rld tota & KTMX & 7731 & 747 & 308488 & 6522 & 336255 & 3771 & 419822 & 416681 & 461988 & 21225 & 6480 \\
\hline
\end{tabular}


9.3 Trade in goods and services

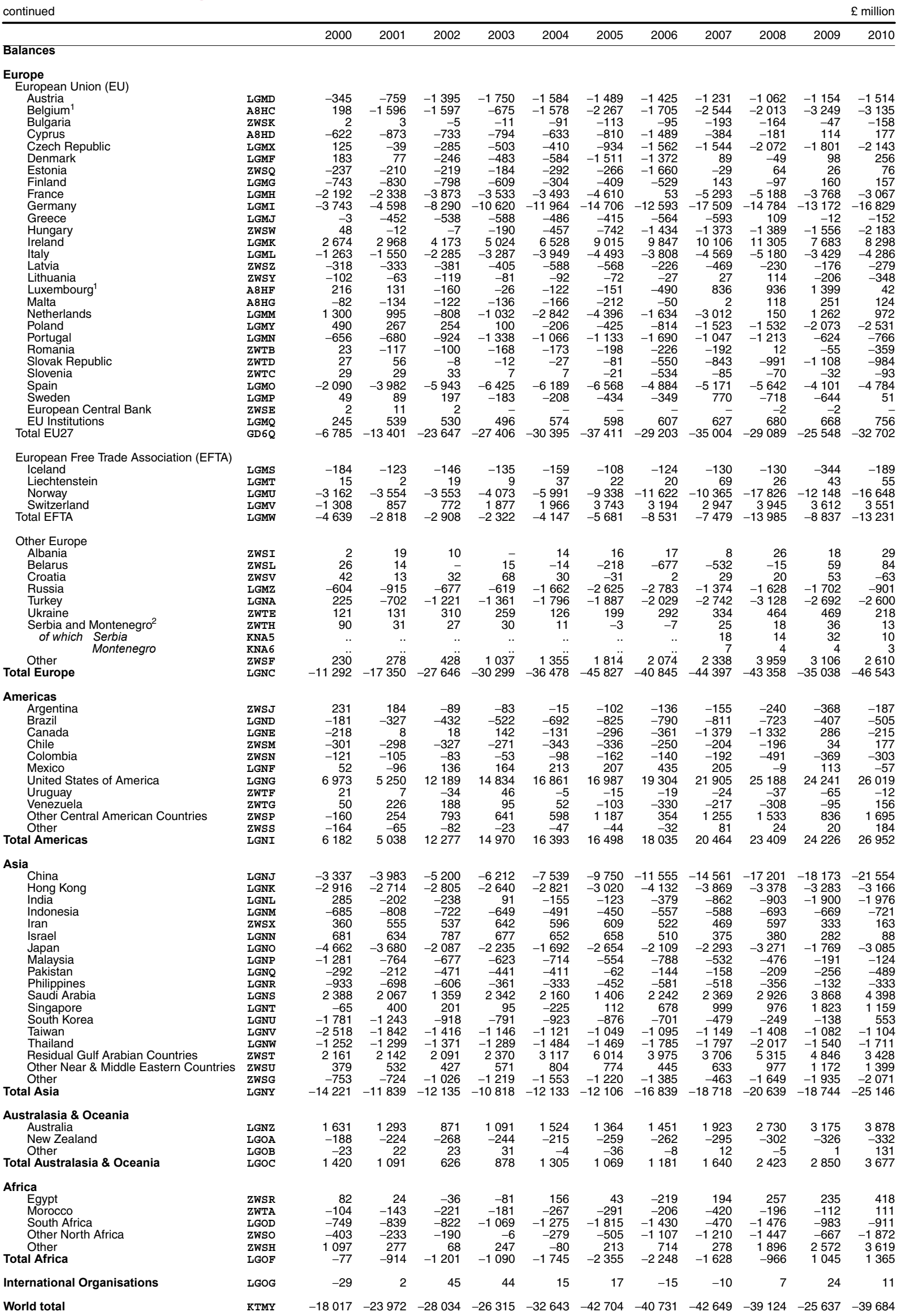

1 Before 1999 only combined BLEU data is available under identifier LGME. 2 Separate Serbia and Montenegro data is available from 2007. 


\subsection{Trade in goods}

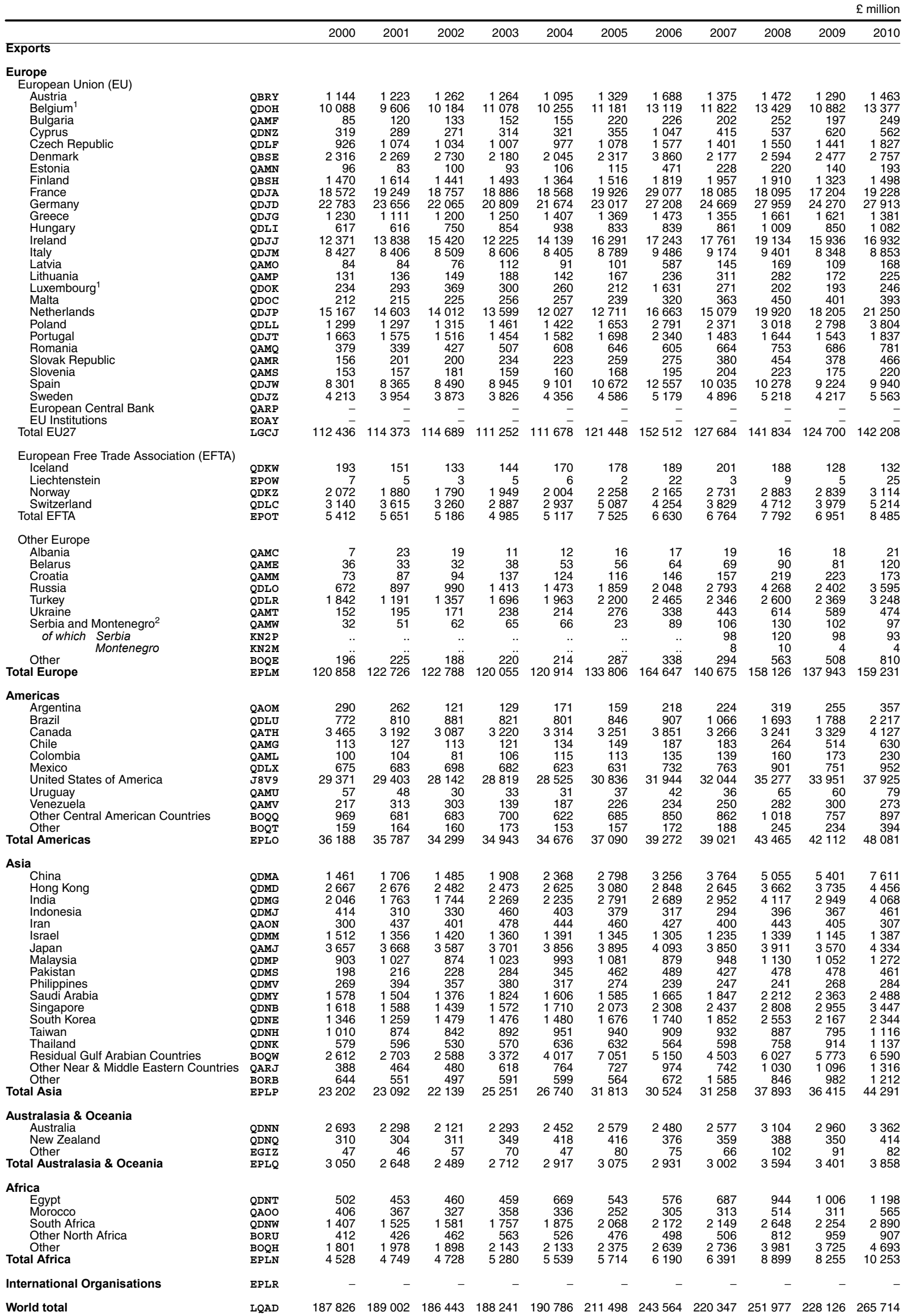

1 Before 1999 only combined BLEU data is available under identifier QBSB. _ 2 Separate Serbia and Montenegro data is available from 2007. 
continued 1. Trade in goods

\begin{tabular}{|c|c|c|c|c|c|c|c|c|c|c|c|c|}
\hline & & 2000 & 2001 & 2002 & 2003 & 2004 & 2005 & 2006 & 2007 & 2008 & 2009 & 201 \\
\hline \multicolumn{13}{|l|}{ Imports } \\
\hline \multirow{2}{*}{\multicolumn{13}{|c|}{$\begin{array}{l}\text { Europe } \\
\text { European Union (EU) }\end{array}$}} \\
\hline & & & & & & 2355 & 2469 & 2776 & 2494 & 2353 & 2280 & \\
\hline Belgium $^{1}$ & 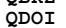 & 10486 & 11636 & 12339 & 12343 & 12786 & 14113 & 15281 & 15016 & 16441 & 15005 & \\
\hline Bulgaria & QAMZ & & 100 & 117 & 126 & 150 & 173 & 198 & 239 & 215 & 183 & \\
\hline $\begin{array}{l}\text { Cyprus } \\
\text { Czech Republic }\end{array}$ & $\begin{array}{l}\text { QDOA } \\
\text { QDLG }\end{array}$ & $\begin{array}{l}205 \\
801\end{array}$ & $\begin{array}{r}238 \\
1099\end{array}$ & $\begin{array}{r}246 \\
1250\end{array}$ & $\begin{array}{r}253 \\
1416\end{array}$ & $\begin{array}{r}208 \\
1290\end{array}$ & $\begin{array}{r}273 \\
1880\end{array}$ & $\begin{array}{l}1712 \\
2952\end{array}$ & $\begin{array}{r}196 \\
2987\end{array}$ & $\begin{array}{r}155 \\
3601\end{array}$ & 123 & \\
\hline $\begin{array}{l}\text { Denmark } \\
\text { Dofic }\end{array}$ & QBSF & 2639 & 2927 & 3604 & $\begin{array}{l}1410 \\
3402\end{array}$ & $\begin{array}{l}3367 \\
3367\end{array}$ & $\begin{array}{l}4416 \\
4480\end{array}$ & 6522 & $\begin{array}{l}2987 \\
3448\end{array}$ & $\begin{array}{l}3001 \\
3937\end{array}$ & $\begin{array}{l}3836 \\
3837\end{array}$ & \\
\hline Estonia & QAND & 312 & & 322 & 264 & 380 & 365 & 2118 & 239 & 151 & 127 & \\
\hline Finland & QBTG & 2764 & 2963 & 2789 & 2663 & 2340 & 2432 & 3068 & 2620 & 2799 & 2113 & \\
\hline France & QDJB & 18674 & 20134 & 20807 & 20388 & 20150 & 22038 & 26720 & 21954 & 23290 & 20439 & \\
\hline Germany & QDJE & 28502 & 30206 & 32450 & 33674 & 35407 & 39176 & 41920 & 44657 & 44891 & 39984 & \\
\hline $\begin{array}{l}\text { Greece } \\
\text { Hungary }\end{array}$ & QDJH & 452 & 485 & 595 & $\begin{array}{r}645 \\
1118\end{array}$ & $\begin{array}{r}665 \\
1581\end{array}$ & $\begin{array}{r}716 \\
186\end{array}$ & $\begin{array}{r}811 \\
2358\end{array}$ & $\begin{array}{r}648 \\
2379\end{array}$ & $\begin{array}{r}665 \\
2541\end{array}$ & 558 & \\
\hline $\begin{array}{l}\text { Hungary } \\
\text { Ireland }\end{array}$ & $\begin{array}{l}\text { QDLJ } \\
\text { ODJK }\end{array}$ & $\begin{array}{r}680 \\
10286\end{array}$ & $\begin{array}{r}707 \\
12190\end{array}$ & $\begin{array}{r}846 \\
13218\end{array}$ & $\begin{array}{l}1118 \\
9919\end{array}$ & $\begin{array}{r}1581 \\
10141\end{array}$ & $\begin{array}{r}1862 \\
10409\end{array}$ & $\begin{array}{r}2358 \\
10716\end{array}$ & $\begin{array}{r}2379 \\
11349\end{array}$ & $\begin{array}{r}2541 \\
12289\end{array}$ & $\begin{array}{r}2540 \\
12430\end{array}$ & \\
\hline Italy & QDJN & 9526 & 9854 & 10673 & 11479 & 12197 & 12685 & 12862 & 13329 & 14194 & 12314 & \\
\hline Latvia & QANE & 407 & 430 & 476 & 517 & 690 & 718 & 798 & 627 & & 339 & \\
\hline Lithuania & QANF & 244 & 229 & 263 & 287 & 270 & 279 & 283 & 309 & 359 & 370 & \\
\hline Luxembourg ${ }^{1}$ & QDOL & 224 & 421 & 753 & $\begin{array}{l}730 \\
186\end{array}$ & 931 & 917 & 2728 & 694 & 835 & 616 & \\
\hline $\begin{array}{l}\text { Malta } \\
\text { Netherlands }\end{array}$ & $\begin{array}{l}\text { QDOD } \\
\text { QDTO } \\
\text { ODTO }\end{array}$ & $\begin{array}{r}128 \\
15397\end{array}$ & $\begin{array}{r}145 \\
15393\end{array}$ & 16148 & $\begin{array}{r}186 \\
16698\end{array}$ & $\begin{array}{l}184 \\
18212\end{array}$ & $\begin{array}{l}179 \\
20463\end{array}$ & $\begin{array}{l}167 \\
22441\end{array}$ & $\begin{array}{r}199 \\
23198\end{array}$ & $\begin{array}{l}136 \\
25979\end{array}$ & $\begin{array}{l}106 \\
.91945\end{array}$ & \\
\hline $\begin{array}{l}\text { Netherlands } \\
\text { Poland }\end{array}$ & $\begin{array}{l}\text { QDJQ } \\
\text { QDLM }\end{array}$ & $\begin{array}{r}15397 \\
911\end{array}$ & $\begin{array}{r}15393 \\
1167\end{array}$ & $\begin{array}{r}16148 \\
1266\end{array}$ & $\begin{array}{r}166988 \\
1546\end{array}$ & $\begin{array}{r}18212 \\
1834\end{array}$ & $\begin{array}{r}20463 \\
2322\end{array}$ & $\begin{array}{r}\begin{array}{r}22441 \\
3664 \\
664\end{array}\end{array}$ & $\begin{array}{r}23198 \\
3699\end{array}$ & $\begin{array}{r}259 / 9 \\
4327\end{array}$ & $\begin{array}{r}11945 \\
4669\end{array}$ & \\
\hline $\begin{array}{l}\text { Portug } \\
\text { Pon }\end{array}$ & QDJU & 1734 & $\begin{array}{l}1624 \\
1624\end{array}$ & $\begin{array}{l}1761 \\
1761\end{array}$ & $\begin{array}{l}1966 \\
1966\end{array}$ & $\begin{array}{l}1927 \\
1927\end{array}$ & 2021 & $\begin{array}{l}3004 \\
3169\end{array}$ & 1512 & $\begin{array}{l}4027 \\
1746\end{array}$ & 1423 & \\
\hline Romania & QANG & 336 & 452 & 519 & 681 & 787 & 802 & 828 & 944 & 811 & 788 & \\
\hline Slovak Republic & QANH & 138 & 174 & 211 & 260 & 263 & 372 & 845 & 1275 & 1633 & 1604 & \\
\hline Slovenia & QANI & 123 & 149 & 173 & 171 & 171 & 204 & 750 & 318 & 320 & 251 & \\
\hline Spain & QDJX & 6150 & 7365 & 9196 & 9254 & 9126 & 11528 & 12011 & 10499 & 10821 & 9570 & 10 \\
\hline $\begin{array}{l}\text { Sweden } \\
\text { European Centra }\end{array}$ & $\begin{array}{l}\text { QDKA } \\
\text { QDARO }\end{array}$ & 4954 & 4669 & 4323 & 4567 & 5123 & 5465 & 6176 & 5305 & 6888 & 5726 & \\
\hline EU Institutions & $\begin{array}{l}\text { QARQ } \\
\text { EOBS }\end{array}$ & & & & & & & & & & & \\
\hline
\end{tabular}

European Free Trade Association (EFTA)

Iceland
Liechtenstein

Norway

Switzerland
Total EFTA

Other Europe

Albania

Belarus
Croatia

Russia

Turkey

Serbia and Montenegro ${ }^{2}$

of which Serbia

Other
Total Europe

Americas

Argentina

Canada

Chile

Colombia

United States of America

Uruguay

Other Central American Countries

Total Americas

Asia

China

Hong Kong

India

Indone
Iran
Israel
Japan

Japan

Makissian

Philippines

Singapore

South Korea

Taiwan

Thailand

Other
Total Asia

Australasia \& Oceania

Australia

New Zea

Total Australasia \& Oceania

Africa

Egypt

South Africa

Other North Africa

Other

International Organisations

World total
Other Near \& Middle Eastern Countries

$\begin{array}{llllllllllll}117577 & 126922 & 136910 & 137329 & 142535 & 158277 & 183874 & 170114 & 181797 & 162676 & 186048\end{array}$

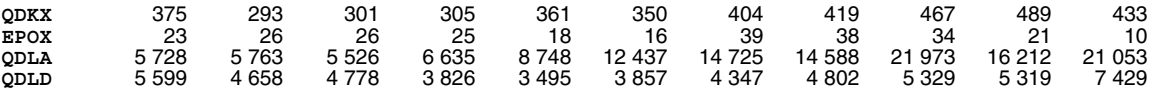
$\begin{array}{lrrrrrrrrrrr}\text { QDLD } & 5599 & 4658 & 4778 & 3826 & 3495 & 3857 & 4347 & 4802 & 5329 & 5319 & 7429 \\ \text { EPOU } & 11725 & 10740 & 10631 & 10791 & 12622 & 16660 & 19515 & 19847 & 27803 & 22041 & 28925\end{array}$

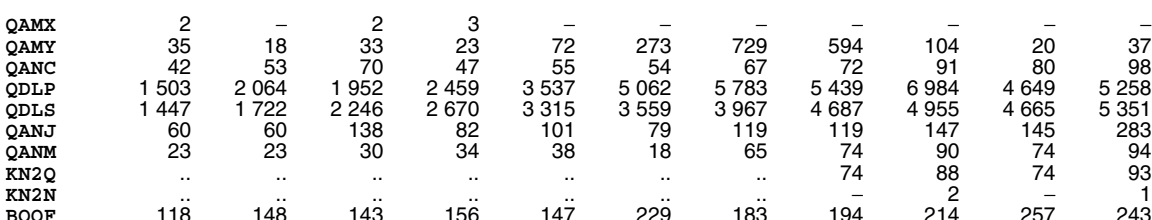

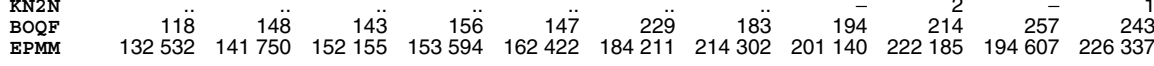

\begin{tabular}{|c|c|c|c|c|c|c|c|c|c|c|c|}
\hline QAOP & 170 & 199 & 238 & 247 & 259 & 273 & 346 & 420 & 568 & 669 & 666 \\
\hline QDLV & 1129 & 1298 & 1383 & 1501 & 1583 & 1777 & 1934 & 2060 & 2658 & 2566 & 3111 \\
\hline QATI & 3 975 & 3630 & 3522 & 3664 & 4172 & 4142 & 4916 & 5791 & 5725 & 4464 & 5770 \\
\hline QANA & 475 & 480 & 479 & 428 & 488 & 492 & 527 & 497 & 586 & 600 & 584 \\
\hline QANB & 238 & 318 & 215 & 224 & 277 & 301 & 301 & 368 & 701 & 583 & 678 \\
\hline QDLY & 607 & 669 & 491 & 477 & 399 & 431 & 427 & 555 & 782 & 757 & 1032 \\
\hline J8VA & 28465 & 29855 & 25234 & 23000 & 22121 & 22049 & 25416 & 25608 & 25540 & 24222 & 26992 \\
\hline QANK & 37 & 37 & 46 & 43 & 41 & 58 & 66 & 71 & 116 & 114 & \\
\hline QANL & 204 & 155 & 179 & 112 & 208 & 391 & 605 & 488 & 615 & 428 & 421 \\
\hline $\mathrm{DQR}$ & 1075 & 624 & 786 & 1020 & 1128 & 1211 & 1411 & 1165 & 1371 & 1249 & 1146 \\
\hline DQU & 286 & 301 & 268 & 224 & 236 & 224 & 301 & 251 & 369 & 352 & \\
\hline ?MO & 36661 & 37566 & 32841 & 30940 & 30912 & 31349 & 36250 & 37274 & 39031 & 36004 & 40867 \\
\hline MB & 4955 & 5905 & 6913 & 8543 & 10626 & 3240 & 15534 & 8881 & 23447 & 24627 & 366 \\
\hline Q̃DME & 6053 & 5878 & 5669 & 5588 & 5852 & 6691 & 7446 & 6989 & 8169 & 7757 & \\
\hline QDMH & 1650 & 1823 & 1801 & 2079 & 2299 & 2770 & 3127 & 3735 & 4533 & 4618 & 581 \\
\hline DMK & 1166 & 1202 & 1090 & 976 & 984 & 920 & 1029 & 1010 & 1219 & 1240 & \\
\hline 100 & 21 & 14 & 20 & 15 & 29 & 25 & 60 & 55 & 69 & 211 & \\
\hline QDMN & 1010 & 919 & 860 & 839 & 913 & 996 & 958 & 1046 & 1172 & 1099 & 15 \\
\hline QAMK & 10124 & 9089 & 8068 & 8086 & 8065 & 8607 & 7834 & 7910 & 8547 & 6670 & 81 \\
\hline DMQ & 2347 & 1976 & $\begin{array}{l}1762 \\
176\end{array}$ & 1897 & 2053 & $\begin{array}{l}1829 \\
\text { - }\end{array}$ & 1917 & 1702 & $\begin{array}{l}1893 \\
\text {. }\end{array}$ & 1665 & \\
\hline DMT & 366 & 426 & 474 & 521 & 556 & 483 & 508 & 493 & 637 & 702 & \\
\hline QDMW & 1179 & 1188 & 968 & 726 & 667 & 722 & 748 & 720 & 637 & 396 & \\
\hline Q̃DMZ & 1006 & 941 & 687 & 728 & 1195 & 1836 & 1277 & 893 & 688 & 610 & 766 \\
\hline QDNC & 2433 & 2087 & 1976 & 2699 & 3431 & 3877 & 3788 & 4086 & 4044 & 3589 & 41 \\
\hline DNF & 3382 & 2751 & 2717 & 2553 & 3069 & 3035 & 3056 & 2990 & 3509 & 2862 & \\
\hline QDNI & 3652 & 2862 & 2445 & 2239 & 2385 & 2262 & 2379 & 2414 & 2631 & 2268 & 3 \\
\hline DNL & 1640 & 1656 & 1587 & 1679 & 1798 & 1747 & 1960 & 2021 & 2454 & 2321 & \\
\hline$Q x$ & 1140 & 112 & 1218 & 1571 & 1737 & 2010 & 2238 & 2137 & 2398 & $2 \varepsilon$ & \\
\hline QARK & 115 & 116 & 170 & 156 & 91 & 202 & 749 & 376 & 435 & 343 & \\
\hline BORD & 1575 & 1622 & 1692 & 1958 & 2207 & 2049 & 2463 & 2643 & 3158 & 3626 & \\
\hline EPMP & 43814 & 41579 & 40117 & 42853 & 47957 & 53301 & 57071 & 60101 & 69640 & 67425 & 81 \\
\hline DNO & 1459 & 1717 & 1645 & 1740 & 1829 & 2059 & 2081 & 2190 & 2384 & 2225 & \\
\hline 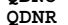 & 53 & 542 & 517 & 547 & 578 & 586 & 602 & 657 & 750 & 819 & \\
\hline 'KF & 129 & 99 & 98 & 126 & 127 & 131 & 130 & 116 & 169 & 168 & \\
\hline MQ & 2123 & 2358 & 2260 & 2413 & 2534 & 2776 & 2813 & 2963 & 3303 & $\begin{array}{l}3212 \\
\end{array}$ & \\
\hline QDNU & 4 & 402 & 409 & 42 & 491 & 335 & 66 & 5 & 647 & 687 & \\
\hline QAOR & & & 45 & & 515 & 414 & 3 & 42 & 440 & & \\
\hline $\mathrm{NX}$ & 2604 & 2895 & 2728 & 2990 & 3321 & 4001 & 3952 & 3143 & 4794 & 3854 & \\
\hline & 757 & 529 & 611 & 589 & 763 & 1007 & 1727 & $17 C$ & 2375 & 1 & \\
\hline & 1498 & 658 & 2532 & 2519 & 2810 & 2888 & 2994 & 3665 & 37 & 30 & \\
\hline MN & 5726 & 6929 & 6737 & 6972 & 7900 & 8645 & 9697 & 9474 & 11999 & 9730 & \\
\hline
\end{tabular}

1 Before 1999 only combined BLEU data is available under indentifier QBSC. 2 Separate Serbia and Montenegro data is available from 2007. 


\begin{tabular}{|c|c|c|c|c|c|c|c|c|c|c|c|c|}
\hline ontinued & & & & & & & & & & & & $£$ mill \\
\hline & & 2000 & 2001 & 2002 & 2003 & 2004 & 2005 & 2006 & 2007 & 2008 & 2009 & 20 \\
\hline Balances & & & & & & & & & & & & \\
\hline $\begin{array}{l}\text { Europe } \\
\text { European } L\end{array}$ & & & & & & & & & & & & \\
\hline $\begin{array}{l}\text { uropean } \\
\text { Austria }\end{array}$ & 3SA & -273 & -662 & -1133 & -1512 & -1260 & -1140 & -1088 & -1119 & -881 & -990 & \\
\hline $\begin{array}{l}\text { Belgium } 1 \\
\text { Bulgaria }\end{array}$ & $\begin{array}{l}\text { QDOJ } \\
\text { QDOJ }\end{array}$ & $\begin{array}{l}-398 \\
-2\end{array}$ & $\begin{array}{l}-2030 \\
\end{array}$ & $\begin{array}{l}-2155 \\
16\end{array}$ & -1265 & -2531 & -2932 & $\begin{array}{l}-2162 \\
168\end{array}$ & -3194 & $\begin{array}{l}-3012 \\
-37\end{array}$ & $\begin{array}{l}-4123 \\
14\end{array}$ & \\
\hline $\begin{array}{l}\text { Bugaria } \\
\text { Cyprus }\end{array}$ & & 114 & $\begin{array}{l}20 \\
51\end{array}$ & $\begin{array}{l}16 \\
25\end{array}$ & $\begin{array}{l}26 \\
61\end{array}$ & $\begin{array}{r}5 \\
113\end{array}$ & $\begin{array}{l}47 \\
82\end{array}$ & $\begin{array}{l}28 \\
-665\end{array}$ & $\begin{array}{l}-37 \\
219\end{array}$ & $\begin{array}{r}37 \\
382\end{array}$ & $\begin{array}{r}14 \\
497\end{array}$ & \\
\hline Czech Republic & H & 125 & -25 & -216 & -409 & -313 & -802 & -1375 & -1586 & -2051 & -1895 & \\
\hline $\mathrm{D}$ & & -323 & -658 & -874 & -1222 & -1322 & -2099 & -2662 & -1271 & -1343 & -1360 & \\
\hline Estc & QANT & -216 & -197 & -222 & -171 & -274 & -250 & -1647 & -11 & 69 & 13 & \\
\hline $\begin{array}{l}\text { Finlan } \\
\text { Franc }\end{array}$ & QBTL & -1294 & -1349 & -1348 & $\begin{array}{l}-1170 \\
-1502\end{array}$ & $\begin{array}{l}-976 \\
-1582\end{array}$ & $\begin{array}{l}-916 \\
-9112\end{array}$ & $\begin{array}{r}-1249 \\
2357\end{array}$ & -663 & -889 & -790 & \\
\hline $\begin{array}{l}\text { France } \\
\text { Germany }\end{array}$ & $\begin{array}{l}\text { QDJC } \\
\text { QDJF }\end{array}$ & $\begin{array}{r}-102 \\
-5719\end{array}$ & $\begin{array}{r}-885 \\
-6550\end{array}$ & $\begin{array}{r}-2050 \\
-10385\end{array}$ & $\begin{array}{r}-1502 \\
-12865\end{array}$ & $\begin{array}{r}-1582 \\
-13733\end{array}$ & $\begin{array}{r}-2112 \\
-16159\end{array}$ & $\begin{array}{r}2357 \\
-14712\end{array}$ & $\begin{array}{r}-3869 \\
-19988\end{array}$ & $\begin{array}{r}-5195 \\
-16932\end{array}$ & $\begin{array}{r}-3235 \\
-15714\end{array}$ & $\begin{array}{r}-23 \\
-185\end{array}$ \\
\hline Greece & QDJI & 778 & 626 & 605 & 605 & 742 & 653 & & & 996 & 1063 & \\
\hline $\mathrm{Hu}$ & QDLK & -63 & -91 & -96 & -264 & -643 & -1029 & -1519 & -1518 & -1532 & -1690 & \\
\hline $\begin{array}{l}\text { |reel } \\
\text { |tal| }\end{array}$ & & $\begin{array}{r}2085 \\
1099\end{array}$ & & 2202 & 2306 & 3998 & 5882 & 6527 & 6412 & 6845 & 3506 & $\begin{array}{r}40 \\
-50\end{array}$ \\
\hline $\begin{array}{l}\text { Ita } \\
\mathrm{La}\end{array}$ & $\begin{array}{l}\text { QDJO } \\
\text { OANU }\end{array}$ & $\begin{array}{r}-1099 \\
-323\end{array}$ & $\begin{array}{r}-1448 \\
-346\end{array}$ & $\begin{array}{l}-2164 \\
-400\end{array}$ & $\begin{array}{l}-2873 \\
-405\end{array}$ & $\begin{array}{r}-3792 \\
-599\end{array}$ & $\begin{array}{l}-3896 \\
-617\end{array}$ & $\begin{array}{l}-3376 \\
-211\end{array}$ & $\begin{array}{l}-4155 \\
-482 \\
-482\end{array}$ & $\begin{array}{l}-4793 \\
-251\end{array}$ & $\begin{array}{r}-3966 \\
-230\end{array}$ & -50 \\
\hline $\mathrm{Lit}$ & QANV & -113 & -93 & -114 & -99 & -128 & -112 & -47 & 2 & -77 & -198 & \\
\hline $\mathrm{urg}^{1}$ & QD & 10 & -128 & -384 & -430 & -671 & -705 & -1097 & -423 & -633 & -423 & \\
\hline $\begin{array}{l}\text { Mal } \\
\text { Net }\end{array}$ & & 84 & $\begin{array}{r}70 \\
790\end{array}$ & 55 & 70 & 73 & $\begin{array}{r}60 \\
7752\end{array}$ & 153 & 184 & 314 & 295 & -51 \\
\hline $\begin{array}{l}\text { Netherlands } \\
\text { Poland }\end{array}$ & $\begin{array}{l}\text { QDJR } \\
\text { ODLN }\end{array}$ & $\begin{array}{r}-230 \\
388\end{array}$ & $\begin{array}{r}-790 \\
130\end{array}$ & $\begin{array}{r}-2136 \\
49\end{array}$ & $\begin{array}{l}-3099 \\
-85\end{array}$ & $\begin{array}{r}-6185 \\
-412\end{array}$ & $\begin{array}{l}-7752 \\
-669\end{array}$ & $\begin{array}{l}-5778 \\
-873\end{array}$ & $\begin{array}{l}-8119 \\
-1328\end{array}$ & $\begin{array}{l}-6059 \\
-1309\end{array}$ & $\begin{array}{l}-3740 \\
-1871\end{array}$ & $\begin{array}{l}-51 \\
-23\end{array}$ \\
\hline Portugal & QDJV & -71 & -49 & -245 & -512 & -345 & -323 & -829 & -29 & -102 & 120 & \\
\hline $\mathrm{Ro}$ & QAOD & 43 & -113 & -92 & -174 & -179 & -156 & -223 & -280 & -58 & -102 & \\
\hline epublic & QAOG & 18 & 27 & -11 & -26 & -40 & -113 & -570 & -895 & -1179 & -1226 & \\
\hline $\begin{array}{l}\text { Slover } \\
\text { Spain }\end{array}$ & QAOH & $\begin{array}{r}30 \\
2151\end{array}$ & 8 & 8 & -12 & -11 & -36 & -555 & -114 & -97 & -76 & \\
\hline $\begin{array}{l}\text { Spain } \\
\text { Sweden }\end{array}$ & QDJY & $\begin{array}{r}2151 \\
-741\end{array}$ & $\begin{array}{l}1000 \\
-715\end{array}$ & $\begin{array}{l}-706 \\
-450\end{array}$ & $\begin{array}{r}-309 \\
741\end{array}$ & -25 & $\begin{array}{l}-856 \\
-879\end{array}$ & $\begin{array}{r}546 \\
-997\end{array}$ & $\begin{array}{l}-464 \\
-409\end{array}$ & $\begin{array}{r}-543 \\
-1670\end{array}$ & $\begin{array}{l}-346 \\
-1509\end{array}$ & \\
\hline $\begin{array}{l}\text { Sweden } \\
\text { Europear }\end{array}$ & QDKV & -141 & -115 & -450 & -141 & -101 & & -997 & -409 & $-16 / 0$ & -1509 & \\
\hline & & 1 & -20 & $\Omega_{0}$ & 2077 & (2) & 20 & 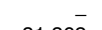 & 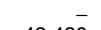 & menges & > & \\
\hline Total E & LGCE & -5141 & -12549 & -22221 & -20011 & -50003 & -36829 & -31362 & -42430 & -39963 & -31910 & \\
\hline European Fre & & & & & & & & & & & & \\
\hline $\begin{array}{l}\text { Icel } \\
\text { Lieg }\end{array}$ & $\underset{\text { EPOY }}{\text { QDKY }}$ & $\begin{array}{l}-182 \\
-16\end{array}$ & $\begin{array}{l}-142 \\
-21\end{array}$ & $\begin{array}{l}-168 \\
-23\end{array}$ & $\begin{array}{l}-161 \\
-20\end{array}$ & $\begin{array}{l}-191 \\
-12\end{array}$ & $\begin{array}{r}-172 \\
-14\end{array}$ & $\begin{array}{l}-215 \\
-17\end{array}$ & $\begin{array}{l}-218 \\
-35\end{array}$ & $\begin{array}{l}-279 \\
-25\end{array}$ & $\begin{array}{l}-361 \\
-16\end{array}$ & \\
\hline Nory & QDLB & $\begin{array}{l}-3656 \\
-3656\end{array}$ & -3883 & -3736 & -4686 & -6744 & -10179 & -12560 & -11857 & -19090 & -13373 & $-17 \mathrm{~s}$ \\
\hline & & -2459 & -1043 & -1518 & -939 & -558 & 1230 & -93 & -973 & -617 & -1340 & -2 \\
\hline Total EFTA & EPOV & -6313 & -5089 & -5445 & -5806 & -7505 & -9135 & -12885 & -13083 & -20011 & -15090 & -202 \\
\hline Other Europ & & & & & & & & & & & & \\
\hline & QANN & 5 & 23 & 17 & 8 & 12 & 16 & 17 & 19 & & 18 & \\
\hline Bela & QANO & 1 & 15 & -1 & 15 & -19 & -217 & -665 & -525 & -14 & 61 & \\
\hline $\mathrm{Cr}$ & QANS & 31 & 34 & 24 & 90 & 69 & 62 & 79 & 85 & 128 & 143 & \\
\hline $\begin{array}{l}\mathrm{Ru} \\
\mathrm{Tu}\end{array}$ & $\begin{array}{l}\text { QDLQ } \\
\text { QDLT }\end{array}$ & $\begin{array}{r}-831 \\
395\end{array}$ & $\begin{array}{r}-1167 \\
-531\end{array}$ & $\begin{array}{l}-962 \\
-889\end{array}$ & $\begin{array}{r}-1046 \\
-974\end{array}$ & $\begin{array}{l}-2064 \\
-1352\end{array}$ & $\begin{array}{l}-3203 \\
-1359\end{array}$ & $\begin{array}{l}-3735 \\
-1502\end{array}$ & $\begin{array}{l}-2646 \\
-2341\end{array}$ & $\begin{array}{l}-2716 \\
-2355\end{array}$ & $\begin{array}{l}-2247 \\
-2296\end{array}$ & $\begin{array}{l}-16 \\
-21\end{array}$ \\
\hline & QAOI & 92 & 135 & 33 & 156 & $\begin{array}{l}-113 \\
-113\end{array}$ & 197 & 219 & 324 & 467 & 444 & \\
\hline Serbia and Mor & QAOL & 9 & 28 & 32 & 31 & 28 & 5 & 24 & 32 & 40 & 28 & \\
\hline of which $S$ & & & & & & & & & 24 & 32 & 24 & \\
\hline Other & $\begin{array}{l}\mathrm{KN} \\
\text { BOS }\end{array}$ & & 77 & & $6 \ddot{4}$ & & & $15 \overline{5}$ & 100 & $\begin{array}{r}8 \\
349\end{array}$ & 251 & \\
\hline Total Europe & EPNM & 11674 & -19024 & -29367 & -33539 & -41508 & -50405 & -49655 & -60465 & -64059 & -56664 & -67 \\
\hline ner & & & & & & & & & & & & \\
\hline & QA & 120 & & -117 & -118 & -88 & -114 & -128 & -196 & -249 & -414 & \\
\hline & & -357 & -488 & -502 & -680 & -782 & -931 & -1027 & -994 & -965 & -778 & \\
\hline & $Q_{B}^{B}$ & -510 & -438 & -435 & -444 & -858 & -891 & -1065 & -2525 & -2484 & -1135 & -16 \\
\hline & & $\begin{array}{l}-362 \\
-138\end{array}$ & $\begin{array}{l}-353 \\
-214\end{array}$ & -134 & $\begin{array}{l}-307 \\
-118\end{array}$ & -354 & $\begin{array}{l}-343 \\
-118\end{array}$ & $\begin{array}{l}-340 \\
-160\end{array}$ & $\begin{array}{l}-314 \\
-209\end{array}$ & $\begin{array}{l}-322 \\
-541\end{array}$ & $\begin{array}{l}-86 \\
-410\end{array}$ & \\
\hline M & & $\begin{array}{r}-138 \\
68\end{array}$ & $\begin{array}{l}-214 \\
14\end{array}$ & $\begin{array}{r}-134 \\
207\end{array}$ & $\begin{array}{r}-118 \\
205\end{array}$ & $\begin{array}{r}-162 \\
224\end{array}$ & $\begin{array}{r}-188 \\
200\end{array}$ & $\begin{array}{r}-166 \\
305\end{array}$ & $\begin{array}{r}-229 \\
208\end{array}$ & $\begin{array}{r}-541 \\
119\end{array}$ & $\begin{array}{r}-410 \\
-6\end{array}$ & \\
\hline es of $A$ & & 906 & -452 & 2908 & 5819 & 6404 & 8787 & 6528 & 6436 & 9737 & 9729 & \\
\hline Ur & & 20 & 11 & -16 & -10 & -10 & -21 & -24 & -30 & -51 & -54 & \\
\hline Ve & & 13 & 158 & 124 & 27 & -21 & -165 & -371 & -238 & -333 & -128 & \\
\hline tral Ar & Bo & $\begin{array}{l}-106 \\
-127\end{array}$ & 57 & -103 & -320 & -506 & -526 & -561 & -303 & -353 & -492 & \\
\hline $\begin{array}{l}\text { Total Americas } \\
\text { Tor }\end{array}$ & $\begin{array}{l}\text { BOQV } \\
\text { EPNO }\end{array}$ & $\begin{array}{l}-127 \\
-473\end{array}$ & $\begin{array}{l}-137 \\
-1779\end{array}$ & $\begin{array}{l}-108 \\
1458\end{array}$ & $\begin{array}{l}-5003 \\
4003\end{array}$ & $\begin{array}{r}-83 \\
3764\end{array}$ & 5741 & $\begin{array}{l}-129 \\
3022\end{array}$ & $\begin{array}{r}-63 \\
1747\end{array}$ & $\begin{array}{r}-124 \\
4434\end{array}$ & $\begin{array}{l}-118 \\
6108\end{array}$ & \\
\hline Asia & & & & & & & & & & & & \\
\hline Chi & $Q D I$ & & & -5428 & -6635 & -8258 & -10442 & -12278 & & -18392 & -19226 & \\
\hline & & -3386 & -3202 & -318 & -3115 & -3227 & -3611 & -4598 & -4344 & -4507 & -4022 & \\
\hline & & 396 & -60 & & 190 & & 21 & -438 & -183 & -416 & -1669 & -17 \\
\hline In & & $\begin{array}{l}-752 \\
279\end{array}$ & -892 & $\begin{array}{l}-760 \\
381\end{array}$ & $\begin{array}{l}-516 \\
463\end{array}$ & $\begin{array}{l}-581 \\
411\end{array}$ & -541 & $\begin{array}{l}-712 \\
-367 \\
\end{array}$ & $\begin{array}{l}-716 \\
345 \\
345\end{array}$ & -823 & $\begin{array}{l}-873 \\
194\end{array}$ & \\
\hline Is & & 502 & $\begin{array}{l}423 \\
437\end{array}$ & $\begin{array}{l}381 \\
560\end{array}$ & $\begin{array}{l}463 \\
521\end{array}$ & $\begin{array}{l}415 \\
478\end{array}$ & $\begin{array}{l}435 \\
349\end{array}$ & $\begin{array}{l}367 \\
347\end{array}$ & $\begin{array}{l}345 \\
189\end{array}$ & 4 & & \\
\hline Ja & & -6467 & -5421 & -4481 & -4385 & -4209 & -4712 & -3741 & -4060 & -4636 & -3100 & -37 \\
\hline M & & -1444 & -949 & -888 & -874 & -1060 & -748 & -1038 & -754 & -763 & -613 & \\
\hline$P$ & & -168 & -210 & -24 & -237 & -211 & & -19 & & -159 & & -3 \\
\hline $\mathrm{Ph}$ & & $\begin{array}{l}-910 \\
570\end{array}$ & -794 & -611 & -346 & -350 & -448 & $\begin{array}{l}-509 \\
-389\end{array}$ & (-4) & -396 & -128 & 17 \\
\hline $\begin{array}{l}\mathrm{Sa} \\
\mathrm{Sin}\end{array}$ & & $\begin{array}{r}572 \\
-815\end{array}$ & $\begin{array}{r}563 \\
-499\end{array}$ & $\begin{array}{r}689 \\
-537\end{array}$ & $\begin{array}{r}1096 \\
-1127\end{array}$ & $\begin{array}{r}411 \\
1701\end{array}$ & $\begin{array}{r}-251 \\
-1804\end{array}$ & $\begin{array}{r}388 \\
-1480\end{array}$ & $\begin{array}{r}954 \\
-1649\end{array}$ & 1524 & 1753 & $\begin{array}{c}17 \\
-6 \\
-6\end{array}$ \\
\hline & & $\begin{array}{l}-815 \\
-2036\end{array}$ & $\begin{array}{l}-499 \\
-1492\end{array}$ & $\begin{array}{l}-531 \\
-1238\end{array}$ & 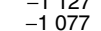 & $\begin{array}{l}-11<1< \\
-1589\end{array}$ & $\begin{array}{l}-1804 \\
-1359\end{array}$ & $\begin{array}{l}-1480 \\
-1316\end{array}$ & $\begin{array}{l}-1649 \\
-1138\end{array}$ & $\begin{array}{l}-1236 \\
-956 \\
-9\end{array}$ & $\begin{array}{l}-634 \\
-695\end{array}$ & $\begin{array}{l}-6 \\
-2 \\
-2\end{array}$ \\
\hline & & $\begin{array}{l}-2036 \\
-2642\end{array}$ & $\begin{array}{l}-1492 \\
-1988\end{array}$ & $\begin{array}{l}-1<00 \\
-1603\end{array}$ & $\begin{array}{l}-1347 \\
-1347\end{array}$ & $\begin{array}{l}-1405 \\
-1434\end{array}$ & $\begin{array}{l}-1322 \\
-1322\end{array}$ & -1470 & $\begin{array}{l}-1100 \\
-1482\end{array}$ & -1744 & -1473 & -20 \\
\hline & & $\begin{array}{l}-<042 \\
-1061\end{array}$ & $\begin{array}{l}-1900 \\
-1060\end{array}$ & $\begin{array}{l}-1057 \\
-1057\end{array}$ & $\begin{array}{l}-1 \text { S } \\
-1109\end{array}$ & $\begin{array}{l}-1434 \\
-1162\end{array}$ & $\begin{array}{l}-13<2 \\
-1115\end{array}$ & $\begin{array}{l}-1470 \\
-1396\end{array}$ & $\begin{array}{l}-1402 \\
-1423 \\
-142\end{array}$ & $\begin{array}{l}-1696 \\
-1696\end{array}$ & $\begin{array}{l}-1473 \\
-1407\end{array}$ & -15 \\
\hline Re & & 1472 & 1579 & 1370 & 1801 & 2280 & 5041 & 2912 & 2366 & 3629 & 2952 & 12 \\
\hline Oth & & 273 & 348 & 310 & 462 & 673 & 525 & 225 & 366 & 595 & 753 & 10 \\
\hline Ton & & -931 & -1071 & -1195 & -1367 & -1608 & -1485 & -1791 & -1058 & -2312 & -2644 & 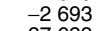 \\
\hline Total Asia & EPNP & 20612 & -18487 & -17978 & -17602 & -21217 & -21488 & -26547 & -28843 & -31747 & -31010 & -37 \\
\hline ust & & & & & & & & & & & & \\
\hline A & QD & 1234 & 581 & 476 & 553 & 623 & $52 C$ & 399 & 387 & 720 & 735 & \\
\hline nd & & -225 & -238 & -206 & -198 & -16 & -17 & -226 & -29 & -3 & -469 & \\
\hline $\begin{array}{l}\text { Other } \\
\text { Total Australasi }\end{array}$ & & & & -41 & $\begin{array}{r}-56 \\
299\end{array}$ & & -51 & $\begin{array}{l}-55 \\
118\end{array}$ & $\begin{array}{r}-50 \\
39\end{array}$ & -6 & $-7,-7>$ & \\
\hline Total Australasia & EPNQ & 927 & 290 & 229 & 299 & 383 & 299 & 118 & 39 & 291 & 189 & \\
\hline Africa & & & & & & & & & & & & \\
\hline & $Q D$ & 92 & & 51 & 31 & 8 & 8 & -86 & 151 & 97 & 319 & \\
\hline & & $\begin{array}{r}-51 \\
-197\end{array}$ & -78 & -130 & -88 & -179 & -162 & -57 & -115 & & & \\
\hline & & $\begin{array}{r}-1197 \\
-345\end{array}$ & $\begin{array}{l}-1370 \\
-103\end{array}$ & -112 & -1233 & $\begin{array}{l}-1446 \\
-237\end{array}$ & -1933 & $\begin{array}{l}-1780 \\
-1200\end{array}$ & 4 & $\begin{array}{l}-2146 \\
-1563\end{array}$ & -16 & \\
\hline Oth & & $\begin{array}{r}-345 \\
303 \\
\end{array}$ & $\begin{array}{l}-103 \\
-680\end{array}$ & $\begin{array}{l}-149 \\
-634\end{array}$ & -26 & -237 & $\begin{array}{l}-531 \\
-513\end{array}$ & $\begin{array}{r}-1229 \\
-355\end{array}$ & $\begin{array}{l}-1196 \\
-1929\end{array}$ & $\begin{array}{r}-1563 \\
238\end{array}$ & & \\
\hline Total Africa & EPNN & -1198 & $\begin{array}{l}-0.00 \\
-2180\end{array}$ & $\begin{array}{l}-634 \\
-2009\end{array}$ & $\begin{array}{l}-1692 \\
-1692\end{array}$ & $\begin{array}{l}-2361 \\
-2361\end{array}$ & $\begin{array}{l}-2931 \\
-290\end{array}$ & $\begin{array}{l}-3507 \\
-3507\end{array}$ & -3083 & $\begin{array}{l}-3100 \\
-3100\end{array}$ & $\begin{array}{r}711 \\
-1475\end{array}$ & -15 \\
\hline International Organisations & EPNR & - & - & - & - & _- & - & - & - & - & - & \\
\hline World total & LQCT & -33030 & -41180 & -47667 & -48531 & -60939 & -68784 & -76569 & -90605 & 181 & -82852 & \\
\hline
\end{tabular}

1 Before 1999 only combined BLEU data is available under identifier QBSD. 2 Separate Serbia and Montenegro data is available from 2007. 


\section{5}

\begin{tabular}{|c|}
\hline \\
\hline \\
\hline Exports \\
\hline $\begin{array}{l}\text { Europe } \\
\text { European Union (EU) }\end{array}$ \\
\hline Austria \\
\hline Belgium $^{1}$ \\
\hline Bulgaria \\
\hline Cyprus \\
\hline Czech Republic \\
\hline Denmark \\
\hline Estonia \\
\hline Finland \\
\hline France \\
\hline Germany \\
\hline Greece \\
\hline Hungary \\
\hline Ireland \\
\hline Italy \\
\hline Latvia \\
\hline Lithuania \\
\hline Luxembourg ${ }^{1}$ \\
\hline Malta \\
\hline Netherlands \\
\hline Poland \\
\hline Portugal \\
\hline Romania \\
\hline Slovak Republic \\
\hline Slovenia \\
\hline Spain \\
\hline Sweden \\
\hline European Central Bank \\
\hline EU Institutions \\
\hline Total EU27 \\
\hline European Free Trade Association (EFTA) \\
\hline Iceland \\
\hline Liechtenstein \\
\hline Norway \\
\hline Switzerland \\
\hline Total EFTA \\
\hline Other Europe \\
\hline Albania \\
\hline Belarus \\
\hline Croatia \\
\hline Russia \\
\hline Turkey \\
\hline Ukraine \\
\hline Serbia and Montenegro ${ }^{2}$ \\
\hline of which Serbia \\
\hline Montenegro \\
\hline Other \\
\hline Total Europe \\
\hline Americas \\
\hline Argentina \\
\hline Brazil \\
\hline Canada \\
\hline Chile \\
\hline Colombia \\
\hline Mexico \\
\hline United States of America \\
\hline Uruguay \\
\hline Venezuela \\
\hline Other Central American Countries \\
\hline Other \\
\hline Total Americas \\
\hline Asia \\
\hline China \\
\hline Hong Kong \\
\hline India \\
\hline Indonesia \\
\hline Iran \\
\hline Israel \\
\hline Japan \\
\hline Malaysia \\
\hline Pakistan \\
\hline Philippines \\
\hline Saudi Arabia \\
\hline Singapore \\
\hline South Korea \\
\hline Taiwan \\
\hline Thailand \\
\hline Residual Gulf Arabian Countries \\
\hline Other Near \& Middle Eastern Countries \\
\hline Other Asian Countries \\
\hline Total Asia \\
\hline Australasia \& Oceania \\
\hline Australia \\
\hline New Zealand \\
\hline Other \\
\hline Total Australasia \& Oceania \\
\hline Africa \\
\hline Egypt \\
\hline Morocco \\
\hline South Africa \\
\hline Other North Africa \\
\hline Othe \\
\hline Total Africa \\
\hline International Organisations \\
\hline World total \\
\hline
\end{tabular}

1 Before 1999 only combined BLEU data is available under indentifier FYVD. 2 Separate Serbia and Montenegro data available from 2007. 


\begin{tabular}{rlrlllllllr}
2000 & 2001 & 2002 & 2003 & 2004 & 2005 & 2006 & 2007 & 2008 & 2009 & 2010 \\
\hline
\end{tabular}

Europe

European Union (EU)

Austria
Belgium
Bulgaria

Bulgaria
Cyprus

Czech Republic

Denmark

Estonia

France

Germany

Greece

reland

Italy

Latvia

Luxembourg

Malta

Poland

Portugal

Slovak Republic

Slovenia

Spain

European Central Bank

EU Institutions

European Free Trade Association (EFTA)

Iceland
Liechtenstein

Norway

Switzerland
Total EFTA

Other Europe

Albania

Belarus

Russia
Turkey
Ukraine

Serbia and Montenegro ${ }^{2}$

of which Serbia

Other

Other
Total Europe

Americas

Argentina

Canada

Chile

Colombia

United States of America

Uruguay

Venezuela

Other

Total Americas

Asia

China

Hong Kong

Indonesia

ran

Japan

Malaysia

Pakistan

Philippines

Singapore

South Korea

Taiwan

Thailand Residual Gulf Arabian Countries

Other Near \& Middle Eastern Countries

Other
asia

Australasia \& Oceania

Australia

New Zer

Total Australasia \& Oceani

Africa

Egypt

Morocco

Other North Africa

Other

Total Africa

International Organisations

World total

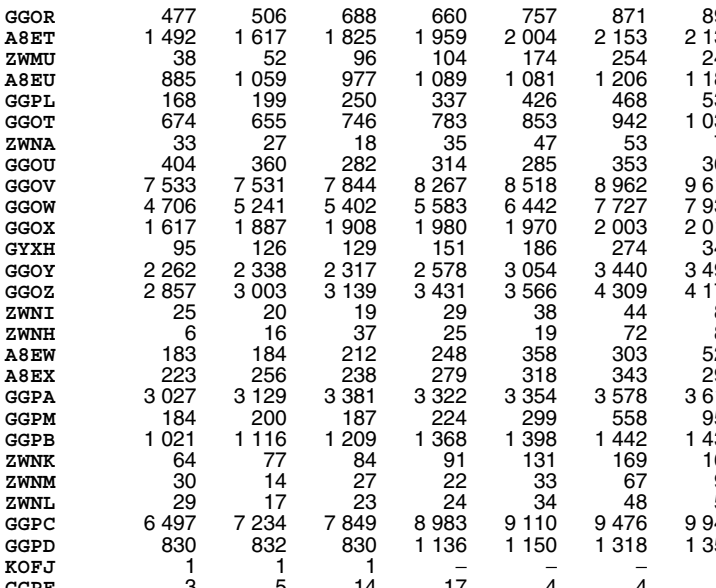

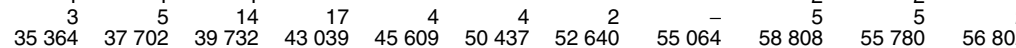

817
2316
333

$\begin{array}{rrr}965 & 908 & 987 \\ 2568 & 2406 & 2319 \\ 394 & 265 & 340\end{array}$

$\begin{array}{lll}394 & 265 & 340 \\ 167 & 934 & 881\end{array}$

$\begin{array}{rrr}465 & 372 & 434 \\ 126 & 1429 & 1294 \\ 55 & 30 & 30\end{array}$

$\begin{array}{rrr}55 & 30 & 30 \\ 402 & 354 & 45\end{array}$

$9916 \quad 9284 \quad 9636$

$\begin{array}{lll}9709 & 8934 & 9127 \\ 144 & 2112 & 1951\end{array}$

$\begin{array}{rrrr}3963 & 4175 & 4255 & 4150\end{array}$

$\begin{array}{rrrr}91 & 68 & 445 & 4350 \\ 96 & 87 & 561 & 94\end{array}$

$\begin{array}{rrrr}96 & 87 & 101 & 118 \\ 497 & 599 & 541 & 1411\end{array}$

$\begin{array}{rrrr}304 & 381 & 327 & 326 \\ 3531 & 3718 & 4224 & 3898\end{array}$

$\begin{array}{llll}3215 & 1297 & 4255 & 1361 \\ 1699 & 1920 & 1515 & 1633\end{array}$

$\begin{array}{rrrr}175 & 214 & 236 & 258 \\ 93 & 119 & 124 & 121\end{array}$

$\begin{array}{rrrr}43 & 66 & 40 & 48 \\ 9991 & 10562 & 9436 & 9482\end{array}$

$\begin{array}{rrrr}1224 & 1424 & 1758 & 1614 \\ - & 2 & 2 & \end{array}$

$\begin{array}{lrrrrrrrrrrr}\text { GGPG } & 75 & 44 & 42 & 46 & 57 & 71 & 98 & 92 & 82 & 130 & 56 \\ \text { GGPH } & 6 & 7 & 1 & 11 & 4 & 4 & 6 & 23 & 8 & 13 & 20 \\ \text { GGPI } & 537 & 647 & 1266 & 710 & 771 & 976 & 1016 & 1049 & 1162 & 1199 & 1092 \\ \text { GGPJ } & 1633 & 1588 & 1677 & 1795 & 1831 & 2355 & 2363 & 2821 & 2667 & 2943 & 3215\end{array}$

$\begin{array}{lllllllllll}1633 & 1588 & 1677 & 1795 & 1831 & 2355 & 2363 & 2821 & 2667 & 2943 & 3215 \\ 2251 & 2286 & 2986 & 2562 & 2663 & 3406 & 3483 & 3985 & 3919 & 4285 & 4383\end{array}$

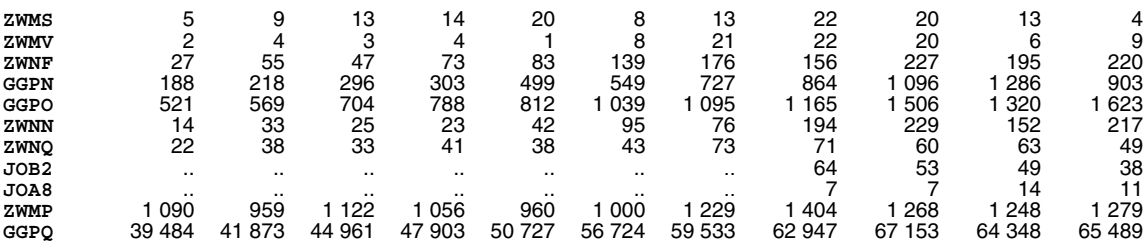

$\begin{array}{rrrrrrr}75 & 74 & 55 & 48 & 69 & 87 & 115 \\ 174 & 231 & 251 & 176 & 217 & 262 & 265 \\ 1105 & 1193 & 1090 & 1003 & 1049 & 1163 & 1346 \\ 35 & 48 & 48 & 43 & 72 & 81 & 39\end{array}$

$\begin{array}{rrrrrrr}35 & 48 & 48 & 43 & 72 & 81 & 39 \\ 39 & 31 & 35 & 32 & 23 & 40 & 46 \\ 283 & 398 & 336 & 312 & 365 & 299 & 274 \\ 13208 & 13509 & 13409 & 14871 & 15311 & 16301 & 16779\end{array}$

$\begin{array}{rrrr}91 & 129 & 100 & 111 \\ 272 & 344 & 323 & 447 \\ 1374 & 1528 & 1279 & 1477 \\ 68 & 49 & 73 & 50\end{array}$

$\begin{array}{rr}68 & 49 \\ 37 & 31\end{array}$

$\begin{array}{rrrr}359 & 457 & 265 & 398 \\ 18069 & 20037 & 19223 & 19200\end{array}$

$\begin{array}{rrrrrrr}6 & 9 & 23 & 4 & 5 & 7 & 12 \\ 46 & 42 & 35 & 34 & 26 & 34 & 31 \\ 1473 & 1407 & 1313 & 1415 & 1882 & 1774 & 2672\end{array}$

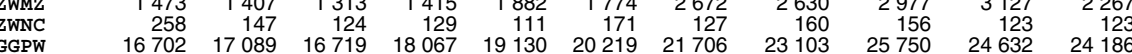

$\begin{array}{rrr}6 & 4 & 26 \\ 37 & 38 & 42 \\ 2630 & 2977 & 3127\end{array}$

$\begin{array}{lrrrrrrrrrrrr}\text { ZWNC } & 258 & 147 & 124 & 129 & 111 & 171 & 127 & 160 & 156 & 123 & 123 \\ \text { GGPW } & 16702 & 17089 & 16719 & 18067 & 19130 & 20219 & 21706 & 23103 & 25750 & 24632 & 24186\end{array}$

2267

GGPX

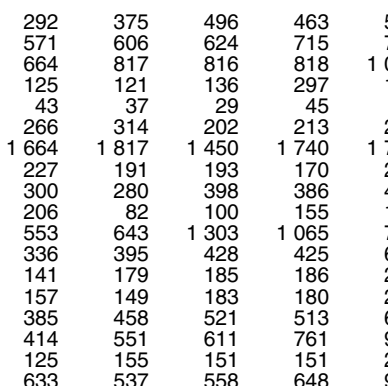

$\begin{array}{rrr}559 & 696 & 8 \\ 721 & 759 & 8 \\ 1089 & 1307 & 15 \\ 100 & 97 & 137 \\ 61 & 42 & \\ 233 & 249 & 2 \\ 1704 & 2259 & 27 \\ 208 & 271 & 2 \\ 431 & 472 & 4 \\ 109 & 142 & 1 \\ 779 & 566 & 6 \\ 661 & 730 & 8 \\ 246 & 254 & 2 \\ 202 & 310 & 2 \\ 641 & 613 & 6 \\ 928 & 1018 & 12 \\ 208 & 199 & 2 \\ 902 & 847 & 10 \\ 9782 & 0831 & 12\end{array}$

$\begin{array}{llrr}1004 & 1326 & 1177 & 1180 \\ 1202 & 1027 & 974 & 1056\end{array}$

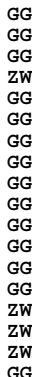

7102

$7 \begin{array}{r}537 \\ 7707\end{array}$

8384

8931

$\begin{array}{rrr}10831 & 12636\end{array}$

$\begin{array}{rrrr}1202 & 1027 & 974 & 1056 \\ 1792 & 2256 & 1942 & 2292\end{array}$

41

302
2917

41
311
3670

74
349

349
3306

$3306 \quad 3627$

$\begin{array}{llll}232 & 244 & 252 & 260 \\ 410 & 394 & 389 & 443 \\ 157 & 196 & 181 & 217\end{array}$

$\begin{array}{llll}157 & 196 & 181 & 217 \\ 742 & 587 & 295 & 249\end{array}$

$\begin{array}{lrrr}940 & 1390 & 1547 & 1803\end{array}$

$\begin{array}{llll}321 & 404 & 364 & 351 \\ 313 & 351 & 270 & 247\end{array}$

$\begin{array}{rrrr}690 & 673 & 630 & 624 \\ 1507 & 2060 & 1973 & 1782 \\ 171 & 220 & 228 & 230\end{array}$

$\begin{array}{rrrr}869 & 220 & 228 & 230 \\ 3733 & 987 & 997 & 1104\end{array}$

$\begin{array}{rrrrrrrrrrrr}\text { GGQN } & 1191 & 1252 & 1431 & 1477 & 1390 & 1869 & 1806 & 1776 & 2046 & 1739 & 2194 \\ \text { GGOO } & 264 & 287 & 337 & 347 & 434 & 501 & 441 & 431 & 369 & 283 & 354\end{array}$

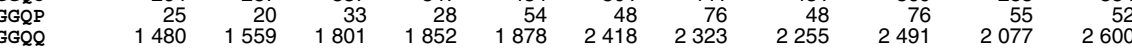

ZWNB

ZWNJ

GWQR

ZWMR

$\begin{array}{rrrrrrr}290 & 367 & 313 & 350 & 344 & 539 & 605 \\ 114 & 147 & 141 & 141 & 149 & 175 & 239 \\ 528 & 598 & 684 & 922 & 909 & 1017 & 1026 \\ 323 & 349 & 263 & 266 & 330 & 349 & 286 \\ 772 & 814 & 1072 & 1289 & 1244 & 1174 & 1278 \\ 2027 & 2275 & 2473 & 2968 & 2976 & 3254 & 3434\end{array}$

$\begin{array}{rrrr}590 & 764 & 741 & 681 \\ 379 & 373 & 254 & 248 \\ 954 & 891 & 883 & 1101 \\ 403 & 429 & 357 & 432 \\ 1308 & 1673 & 1871 & 1472 \\ 3634 & 4130 & 4106 & 3934\end{array}$

$\begin{array}{lllllll}80 & 62 & 40 & 29 & 37 & 43 & 57\end{array}$

57

43

15

30

\begin{tabular}{lllllllllll}
66875 & 70565 & 74378 & 79750 & 84530 & 93489 & 99689 & 105729 & 115830 & 110247 & 112304 \\
\hline
\end{tabular}

1 Before 1999 only combined BLEU data is available under identifier GGOS. 2 Separate Serbia and Montenegro data is available from 2007. 


\subsection{Tratein iserice \\ continued}

\begin{tabular}{llllllllllr}
2000 & 2001 & 2002 & 2003 & 2004 & 2005 & 2006 & 2007 & 2008 & 2009 & 2010 \\
\hline
\end{tabular}

Balances

Europe

European Union (EU)

Austria

Bulgaria

Cyprus

Denmark

Estonia
Finland

France

Germany

Greece

Ireland

Italy

Latvia

Luxembourg ${ }^{1}$

Malta

Netherlands

Portugal

Romania

Slovenia

Spain

European Central Bank

EU Institutions

European Free Trade Association (EFTA)

Iceland
Liechtenstein

Norway

Switzerland
Total EFTA

Other Europe

Albania

Belarus

Russia
Turkey
Ukraine

Serbia and Montenegro ${ }^{2}$

of which Serbia

Other

Other
Total Europe

Montenegro

Americas

Argentina

Canada

Chile

Molombia

United States of Americ

Uruguay

Venezuela

Other

Total Americas

Asia

China

Hong Kong

Indonesia

Iran

srael

Malaysia

Pakistan

Philippines

Singapore

South Korea

Taiwan

Thailand Gulf Arabian Countries

Other Near \& Middle Eastern Countries Other

Total Asia

Australasia \& Oceania

$$
\begin{aligned}
& \text { Australia } \\
& \text { New Zealand }
\end{aligned}
$$

Other
Total Australasia \& Oceania

Africa

Egypt

South Africa

Other North Africa

Other

Total Africa

International Organisations

World total

\begin{tabular}{|c|c|c|c|c|c|c|c|c|c|c|c|}
\hline GGQW & -72 & -97 & -262 & -238 & -324 & -349 & -337 & -112 & -181 & -164 & -344 \\
\hline $\begin{array}{l}\text { A8HH } \\
\text { ZWTO }\end{array}$ & $\begin{array}{r}596 \\
4\end{array}$ & $\begin{array}{r}434 \\
-17\end{array}$ & $\begin{array}{l}558 \\
-21\end{array}$ & $\begin{array}{l}590 \\
-37\end{array}$ & $\begin{array}{r}953 \\
-96\end{array}$ & $\begin{array}{r}665 \\
-160\end{array}$ & $\begin{array}{r}457 \\
-123\end{array}$ & $\begin{array}{r}650 \\
-156\end{array}$ & 999 & 874 & $\begin{array}{r}569 \\
-176\end{array}$ \\
\hline A8HI & $\begin{array}{r}4 \\
-736\end{array}$ & $\begin{array}{r}-11 \\
-924\end{array}$ & -758 & $\begin{array}{r}-31 \\
-855\end{array}$ & $\begin{array}{r}-746 \\
-746\end{array}$ & $\begin{array}{l}-100 \\
-892\end{array}$ & $\begin{array}{l}-1100 \\
-824\end{array}$ & $\begin{array}{l}-1503 \\
-603\end{array}$ & $\begin{array}{l}-201 \\
-563\end{array}$ & $\begin{array}{r}-61 \\
-383\end{array}$ & $\begin{array}{l}-116 \\
-279\end{array}$ \\
\hline GGRQ & & -14 & -69 & -94 & -97 & -132 & -187 & 42 & -21 & 94 & 18 \\
\hline GGQY & 506 & 735 & 628 & 739 & 738 & 588 & 1290 & 1360 & 1294 & 1458 & 1616 \\
\hline ZWTU & -21 & -13 & 3 & -13 & -18 & -16 & -13 & -18 & -5 & 13 & 49 \\
\hline GGQZ & 551 & 519 & 550 & 561 & 672 & 507 & 720 & 806 & 792 & 950 & 823 \\
\hline GGRA & -2090 & -1453 & -1823 & -2031 & -1911 & -2498 & -2304 & -1424 & 7 & -533 & -723 \\
\hline GGRB & 1976 & 1952 & 2095 & 2245 & 1769 & 1453 & 2119 & 2479 & 2148 & 2542 & 1673 \\
\hline GGRC & -781 & -1078 & -1143 & -1193 & -1228 & -1068 & -1226 & -1300 & -887 & -1075 & -832 \\
\hline GYXT & 111 & 79 & 89 & 74 & 186 & 287 & 85 & 145 & 143 & 134 & -14 \\
\hline GGRD & 589 & 1320 & 1971 & 2718 & 2530 & 3133 & 3320 & 3694 & 4460 & 4177 & 4239 \\
\hline GGRE & -164 & -102 & -121 & -414 & -157 & -59 & -432 & -414 & -387 & 537 & 801 \\
\hline ZWUC & 5 & 13 & 19 & - & 11 & 49 & -15 & 13 & 21 & 54 & \\
\hline ZWUB & 11 & 30 & -5 & 18 & 36 & 40 & 20 & 25 & 191 & -8 & -22 \\
\hline A8HK & 206 & 259 & 224 & 404 & 549 & 554 & 607 & 1259 & 1569 & 1822 & 733 \\
\hline A8HL & -166 & -204 & -177 & -206 & -239 & -272 & -203 & -182 & -196 & -44 & 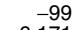 \\
\hline GGRF & 1530 & 1785 & 1328 & 2067 & 3343 & 3356 & 4144 & 5107 & 6209 & 5002 & 6171 \\
\hline GGRR & 102 & 137 & 205 & 185 & 206 & 244 & 59 & -195 & -223 & -202 & -229 \\
\hline GGRG & -585 & -631 & -679 & -826 & -721 & -810 & -861 & -1018 & -1111 & -744 & -8 \\
\hline ZWUE & -20 & -4 & -8 & 6 & 6 & -42 & -3 & 88 & 70 & 47 & 10 \\
\hline ZWUG & 9 & 29 & 3 & 14 & 13 & 3 & 2( & 52 & 18 & 118 & \\
\hline ZWUF & -1 & 21 & 25 & 19 & 18 & 15 & 21 & 29 & 27 & 44 & \\
\hline GGRH & -4241 & -4982 & -5237 & -6116 & -6164 & -5712 & -5430 & -4707 & -5099 & -3755 & -4343 \\
\hline GGRI & 790 & 804 & 647 & 558 & 559 & 445 & 648 & 1179 & 952 & 865 & 12 \\
\hline ZWTI & & 11 & & & & & & & ? & -2 & \\
\hline GGRJ & $24 \overline{5}$ & 539 & 530 & 496 & 574 & 598 & 607 & 627 & 680 & 668 & \\
\hline GD6R & -1644 & -852 & -1426 & -1329 & 462 & -582 & 2159 & 7426 & 10874 & 12428 & 1113 \\
\hline GGRL & -2 & 19 & 26. & 26 & 32 & 64 & 91 & 88 & 149 & 17 & \\
\hline GGRM & 31 & 23 & 19. & 29 & 49 & & 37 & 104 & & 59 & \\
\hline GGRN & 494 & 329 & 183 & 613 & 753 & 84 & 938 & 1492 & 12 & 1225 & 12 \\
\hline GGRO & 1151 & 1900 & 229 & 2816 & 2524 & 2513 & 3288 & 3920 & 4562 & 4952 & 57 \\
\hline GGRP & 1674 & 2271 & 2537 & 3484 & 3358 & 3454 & 4354 & 5604 & 6026 & 6253 & 72 \\
\hline
\end{tabular}

ZWTM
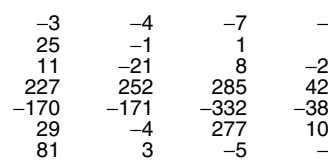

$\begin{array}{rr}-8 & 2 \\ - & 5 \\ -22 & -39 \\ 427 & 402 \\ -387 & -444 \\ 103 & 13 \\ -1 & -17\end{array}$

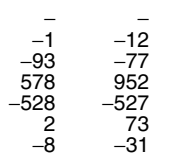

$\begin{array}{rr}-11 & 10 \\ -7 & -1 \\ -56 & -108 \\ 1272 & 1088 \\ -401 & -773 \\ 10 & -3 \\ -7 & -22\end{array}$

$\begin{array}{rr}- & 8 \\ -2 & 1 \\ -90 & -138 \\ 545 & 762 \\ -396 & -497 \\ 25 & 27 \\ 8 & 10 \\ 8 & 10\end{array}$

$\begin{array}{lrrrrrrrrrrr}\text { JOB8 } & 152 & 201 & \dot{383} & 973 & \dot{1} 288 & 1756 & 1919 & 2238 & 3610 & 285 \overline{5} & 2043 \\ \text { ZWTJ } & 382 & 1674 & 1721 & 3240 & 5030 & 4578 & 8810 & 16068 & 20701 & 21626 & 20563\end{array}$

ZWT
GG
GG
ZW
ZW
GG
GG
ZW
ZW
ZW
ZW
GG

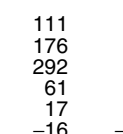

$\begin{array}{rrrrrrrrrr}121 & 28 & 35 & 73 & 12 & -8 & 41 & 9 & 46 & 122 \\ 161 & 70 & 158 & 90 & 106 & 237 & 183 & 242 & 371 & 389 \\ 446 & 453 & 586 & 727 & 595 & 704 & 1146 & 1152 & 1421 & 1428 \\ 55 & 39 & 36 & 11 & 7 & 90 & 110 & 126 & 120 & 131 \\ 109 & 51 & 65 & 64 & 26 & 26 & 37 & 50 & 41 & 145 \\ -110 & -71 & -41 & -11 & 7 & 130 & -3 & -128 & 119 & 23 \\ 5702 & 9281 & 9015 & 10457 & 8200 & 12776 & 15469 & 15451 & 14512 & 15086 \\ -4 & -18 & 56 & 5 & 6 & 5 & 11 & 14 & -11 & 12 \\ 68 & 64 & 68 & 73 & 62 & 41 & 21 & 25 & 33 & 304 \\ 197 & 896 & 961 & 1104 & 1713 & 915 & 1558 & 1886 & 1328 & 1944 \\ 72 & 26 & 28 & 36 & 23 & 97 & 144 & 148 & 138 & 154 \\ 6817 & 10819 & 10967 & 12629 & 10757 & 15013 & 18717 & 18975 & 18118 & 19738\end{array}$

ZWTT
ZWTW
GGSB

$6067 \quad 570$

$\begin{array}{rrr}1 & -4 \\ 37 & 68 \\ -54 & 197 \\ -37 & 72 & \\ 6655 & 6817 & 108\end{array}$

$\begin{array}{lrrrrrrrrrrr}\text { GGSC } & 157 & 216 & 228 & 423 & 719 & 692 & 723 & 556 & 1191 & 1053 & 1472 \\ \text { GGSD } & 470 & 488 & 382 & 475 & 406 & 591 & 466 & 475 & 1129 & 739 & 550 \\ \text { GGSE } & -111 & -142 & -181 & -99 & -91 & -144 & 59 & -79 & -487 & -231 & -232 \\ \text { GGSF } & 67 & 84 & 38 & -133 & 90 & 91 & 155 & 128 & 130 & 204 & 206 \\ \text { ZWUA } & 81 & 132 & 156 & 179 & 181 & 174 & 155 & 124 & 223 & 139 & 62 \\ \text { GGSG } & 179 & 197 & 227 & 156 & 174 & 309 & 163 & 186 & 213 & 236 & 267 \\ \text { GGSH } & 1805 & 1741 & 2394 & 2150 & 2517 & 2058 & 1632 & 1767 & 1365 & 1331 & 688 \\ \text { GGSI } & 163 & 185 & 211 & 251 & 346 & 194 & 250 & 222 & 287 & 422 & 441 \\ \text { GGSJ } & -124 & -2 & -225 & -204 & -200 & -41 & -125 & -92 & -50 & -32 & -141 \\ \text { GGSK } & -23 & 96 & 5 & -15 & 17 & -4 & -72 & -45 & 40 & -4 & -92 \\ \text { GGSL } & 1816 & 1504 & 670 & 1246 & 1749 & 1657 & 1854 & 1415 & 1402 & 2115 & 2676 \\ \text { GGSM } & 750 & 899 & 738 & 1222 & 1496 & 1916 & 2158 & 2648 & 2212 & 2457 & 1858 \\ \text { GGSN } & 255 & 249 & 320 & 286 & 666 & 483 & 615 & 659 & 707 & 557 & 772 \\ \text { GGSO } & 124 & 146 & 187 & 201 & 313 & 273 & 375 & 333 & 336 & 391 & 898 \\ \text { GGSP } & -191 & -239 & -314 & -180 & -322 & -354 & -389 & -374 & -321 & -133 & -165 \\ \text { ZWTX } & 689 & 563 & 721 & 569 & 837 & 973 & 1063 & 1340 & 1686 & 1894 & 2225 \\ \text { ZWTY } & 106 & 184 & 117 & 109 & 131 & 249 & 220 & 267 & 382 & 419 & 380 \\ \text { ZWTK } & 178 & 347 & 169 & 148 & 55 & 265 & 406 & 595 & 663 & 709 & 622 \\ \text { GGSR } & 6391 & 6648 & 5843 & 6784 & 9084 & 9382 & 9708 & 10125 & 11108 & 12266 & 12487\end{array}$

$\begin{array}{rrrrrrrrrrrr}\text { GGSS } & 397 & 712 & 395 & 538 & 901 & 844 & 1052 & 1536 & 2010 & 2440 & 2831 \\ \text { GGST } & 37 & 14 & -62 & -46 & -55 & -89 & -36 & 3 & 60 & 143 & 98\end{array}$

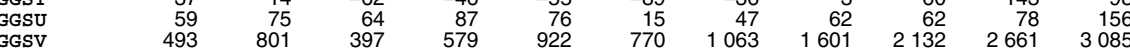

1 Before 1999 only combined BLEU data is available under identifier GGQX..

$\begin{array}{lr}-87 & -11 \\ -91 & -93 \\ 325 & 164 \\ -41 & \\ 702 & 62 \\ 808 & 602\end{array}$

$\begin{array}{rr}-112 & -22 \\ -93 & -88 \\ 164 & 17 \\ 20 & -42 \\ 623 & 597 \\ 602 & 616\end{array}$

$\begin{array}{rr}-22 & -165 \\ -88 & -129 \\ 71 & 118 \\ -42 & 2 \\ 57 & 72 \\ 616 & 57\end{array}$

-344
569
-176
-279
18
1616
49
823
-723
1673
-832
-14
4239
801
-7
-22
733
-99
6171
-229
-854
101
170
44
-4343
1297
756
11138

ZWTV

ZWUD

ZWTS

$\begin{array}{rr}-10 & -27 \\ -53 & -65 \\ 448 & 531 \\ -58 & -130 \\ 794 & 957 \\ 1121 & 1\end{array}$

GGSZ

$-29$

$45 \quad 44$

15

$\begin{array}{rrrr}43 & -40 & -84 & -121 \\ -305 & -270 & -87 & -111 \\ 524 & 670 & 617 & 622 \\ -14 & 116 & 213 & 72 \\ 1207 & 1658 & 1861 & 2432 \\ 1455 & 2134 & 2520 & 2894\end{array}$ 


\subsection{Income}

\begin{tabular}{|c|}
\hline \\
\hline \\
\hline Credits \\
\hline $\begin{array}{l}\text { Europe } \\
\text { European Union (EU) }\end{array}$ \\
\hline Austria \\
\hline Belgium $^{1}$ \\
\hline Bulgaria \\
\hline Cyprus \\
\hline Czech Republic \\
\hline Denmark \\
\hline Estonia \\
\hline Finland \\
\hline France \\
\hline Germany \\
\hline Greece \\
\hline Hungary \\
\hline Ireland \\
\hline Italy \\
\hline Latvia \\
\hline Lithuania \\
\hline Luxembourg $^{1}$ \\
\hline Malta \\
\hline Netherlands \\
\hline Poland \\
\hline Portugal \\
\hline Romania \\
\hline Slovak Republic \\
\hline Slovenia \\
\hline Spain \\
\hline Sweden \\
\hline European Central Bank \\
\hline $\begin{array}{l}\text { EU Institutions } \\
\text { - }\end{array}$ \\
\hline Total EU27 \\
\hline European Free Trade Association (EFTA) \\
\hline $\begin{array}{l}\text { Iceland } \\
\text { Liechtenstein }\end{array}$ \\
\hline $\begin{array}{l}\text { Liechtenstein } \\
\text { Norway }\end{array}$ \\
\hline $\begin{array}{l}\text { Norway } \\
\text { Switzerland }\end{array}$ \\
\hline Switzerland \\
\hline Total EFTA \\
\hline Other Europe \\
\hline Albania \\
\hline Belarus \\
\hline Croatia \\
\hline Russia \\
\hline Turkey \\
\hline Ukraine \\
\hline Serbia and Montenegro ${ }^{2}$ \\
\hline of which Serbia \\
\hline Montenegro \\
\hline $\begin{array}{l}\text { Other } \\
\text { Total Eurone }\end{array}$ \\
\hline Total Europe \\
\hline Americas \\
\hline Argentina \\
\hline Brazil \\
\hline Canada \\
\hline Chile \\
\hline Colombia \\
\hline Mexico \\
\hline United States of America \\
\hline Uruguay \\
\hline Venezuela \\
\hline Other Central American Countries \\
\hline Other \\
\hline Total Americas \\
\hline Asia \\
\hline China \\
\hline Hong Kong \\
\hline India \\
\hline Indonesia \\
\hline Ir \\
\hline Israel \\
\hline Japa \\
\hline Malaysia \\
\hline an \\
\hline Philippines \\
\hline Saudi Arabia \\
\hline Sing \\
\hline Korea \\
\hline Taiws \\
\hline Thailand \\
\hline Residual Gulf Arabian Countries \\
\hline Other Near \& Middle Eastern Countries \\
\hline Other \\
\hline Total Asia \\
\hline Australasia \& $\mathrm{C}$ \\
\hline \\
\hline New Zealand \\
\hline Other \\
\hline Total Australasia \& Oceania \\
\hline Africa \\
\hline Egypt \\
\hline Morocco \\
\hline So \\
\hline Other North Africa \\
\hline Other \\
\hline Total Africa \\
\hline International Organisations \\
\hline World total \\
\hline
\end{tabular}

1 Before 1999 only combined BLEU data is available under identifier CTFK. 2 Separate Serbia and Montenegro data is available from 2007. 


\subsection{Income}

\begin{tabular}{|c|}
\hline continued \\
\hline \\
\hline Debits \\
\hline $\begin{array}{l}\text { Europe } \\
\text { European Union (EU) }\end{array}$ \\
\hline Austria \\
\hline Belgium ${ }^{1}$ \\
\hline Bulgaria \\
\hline Cyprus \\
\hline Czech Republic \\
\hline Denmark \\
\hline Estonia \\
\hline Finland \\
\hline France \\
\hline Germany \\
\hline Greece \\
\hline Hungary \\
\hline Ireland \\
\hline Italy \\
\hline Latvia \\
\hline Lithuania \\
\hline Luxembourg ${ }^{1}$ \\
\hline Malta \\
\hline Netherlands \\
\hline Poland \\
\hline Portugal \\
\hline Romania \\
\hline Slovak Republic \\
\hline Slovenia \\
\hline Spain \\
\hline Sweden \\
\hline European Central Bank \\
\hline $\begin{array}{l}\text { EU Institutions } \\
\text { Total EU27 }\end{array}$ \\
\hline Iotal EU2r \\
\hline European Free Trade Association (EFTA) \\
\hline $\begin{array}{l}\text { Iceland } \\
\text { Liechtenstein }\end{array}$ \\
\hline Liechtenstein \\
\hline Norway \\
\hline Switzerland \\
\hline Total EFTA \\
\hline Other Europe \\
\hline Albania \\
\hline Belarus \\
\hline Croatia \\
\hline Russia \\
\hline Turkey \\
\hline Ukrai \\
\hline Serbia and Montenegro ${ }^{2}$ \\
\hline of which Serbia \\
\hline Mont \\
\hline $\begin{array}{l}\text { Other } \\
\text { Total Europe }\end{array}$ \\
\hline Total Europe \\
\hline Americas \\
\hline $\begin{array}{l}\text { Argentina } \\
\text { Brazil }\end{array}$ \\
\hline Brazil \\
\hline Canada \\
\hline Chile \\
\hline Colombia \\
\hline Mexico \\
\hline United States of America \\
\hline Uruguay \\
\hline Venezuela \\
\hline Other Central American Countries \\
\hline Other \\
\hline Total Americas \\
\hline Asia \\
\hline China \\
\hline Hong Kong \\
\hline Indi \\
\hline Indonesia \\
\hline Ire \\
\hline Israel \\
\hline Japan \\
\hline Malaysia \\
\hline $\mathrm{Pa}$ \\
\hline Philippines \\
\hline Saudi Arabia \\
\hline Singapore \\
\hline Korea \\
\hline Tai \\
\hline Thailand \\
\hline Residual Gulf Arabian Countries \\
\hline Other Near \& Middle Eastern Countries \\
\hline Other \\
\hline Total Asia \\
\hline \& Oceania \\
\hline Australia \\
\hline New Zealand \\
\hline \\
\hline Total Australasia \\
\hline \\
\hline Egypt \\
\hline Morocco \\
\hline South Africa \\
\hline Other North Africa \\
\hline Other \\
\hline Total Africa \\
\hline International Organisations \\
\hline World total \\
\hline
\end{tabular}




\begin{tabular}{|c|c|}
\hline Balances & \\
\hline Balances & \\
\hline $\begin{array}{l}\text { Europe } \\
\text { European Union (EU) }\end{array}$ & \\
\hline Austria & CUHA \\
\hline Belgium 1 & AA3A \\
\hline Bulgaria & ZXAN \\
\hline Cyprus & AA3B \\
\hline Czech Republic & LEPV \\
\hline Denmark & LEQW \\
\hline Estonia & ZXAP \\
\hline Finland & LEUI \\
\hline France & LEUR \\
\hline Germany & LEQN \\
\hline Greece & LEVA \\
\hline Hungary & BFKT \\
\hline Ireland & BFML \\
\hline Italy & BFOI \\
\hline Latvia & ZXAQ \\
\hline Lithuania & ZXAR \\
\hline Luxembourg ${ }^{1}$ & AA3E \\
\hline Malta & AA3 F \\
\hline Netherlands & BFQK \\
\hline Poland & BFS̃D \\
\hline Portugal & BFSM \\
\hline Romania & zXAS \\
\hline Slovak Republic & ZXAT \\
\hline Slovenia & ZXAU \\
\hline Spain & LESY \\
\hline Sweden & BFTN \\
\hline European Central Bank & ZXAK \\
\hline EU Institutions & CSFM \\
\hline Total EU27 & G97D \\
\hline European Free Trade Association (EFTA) & \\
\hline Iceland & BFNU \\
\hline Liechtenstein & BFPU \\
\hline Norway & BFQT \\
\hline Switzerland & LEPD \\
\hline Total EFTA & CTFV \\
\hline Other Europe & \\
\hline Albania & ZXAL \\
\hline Belarus & ZXAM \\
\hline Croatia & ZXAO \\
\hline Russia & BFSV \\
\hline Turkey & BFUO \\
\hline Ukraine & ZXAV \\
\hline Serbia and Montenegro, ${ }^{2}$ & BFWH \\
\hline of which Serbia & KNB3 \\
\hline Montenegro & KNB4 \\
\hline Other & LEWB \\
\hline Total Europe & LERF \\
\hline Americas & \\
\hline Argentina & $\operatorname{zXAX}$ \\
\hline Brazil & LENT \\
\hline Canada & LEOU \\
\hline Chile & ZXAY \\
\hline Colombia & zXAZ \\
\hline Mexico & BFPS \\
\hline United States of America & BFVG \\
\hline Uruguay & ZXBA \\
\hline Venezuela & zXBB \\
\hline Other Central American Countries & JISR \\
\hline Other & LEVU \\
\hline Total Americas & LESP \\
\hline Asia & \\
\hline China & LEPM \\
\hline Hong Kong & BF JW \\
\hline India & BFND \\
\hline Indonesia & BFLR \\
\hline Iran & $\mathrm{ZXBC}$ \\
\hline Israel & BFMU \\
\hline Japan & BFOR \\
\hline Málaysia & $\mathrm{BFQB}$ \\
\hline Pakistan & BFRU \\
\hline Philippines & BFRL \\
\hline Saudi Arabia & BFTE \\
\hline Singapore & BFTW \\
\hline South Korea & BFPA \\
\hline Taiwan & BFUX \\
\hline Thailand & BFUF \\
\hline Residual Gulf Arabian Countries & JITS \\
\hline Other Near \& Middle Eastern Countries & ZXBD \\
\hline Other & LEWK \\
\hline Total Asia & LETH \\
\hline Australasia \& Oceania & \\
\hline Australia & CYAA \\
\hline New Zealand & BFRC \\
\hline Other & LEVS \\
\hline Total Australasia \& Oceania & LETZ \\
\hline Africa & \\
\hline Egypt & $\mathrm{ZXBF}$ \\
\hline Morocco & $\mathrm{ZXBG}$ \\
\hline South Africa & BFWZ \\
\hline Other North Africa & JIRT \\
\hline Other & LEWT \\
\hline Total Africa & LERX \\
\hline International Organisations & CTFD \\
\hline World total & HMBP \\
\hline
\end{tabular}

1 Before 1999 only combined BLEU data is available under identifier CTFM. 2 Separate Serbia and Montenegro data is available from 2007. 


\begin{tabular}{|c|}
\hline \\
\hline \\
\hline Credits \\
\hline $\begin{array}{l}\text { Europe } \\
\text { European Union (EU) }\end{array}$ \\
\hline Austria \\
\hline Belgium ${ }^{1}$ \\
\hline Bulgaria \\
\hline Cyprus \\
\hline Czech Republic \\
\hline Denmark \\
\hline Estonia \\
\hline Finland \\
\hline France \\
\hline Germany \\
\hline Greece \\
\hline Hungary \\
\hline Ireland \\
\hline Italy \\
\hline Latvia \\
\hline Lithuania \\
\hline Luxembourg $^{1}$ \\
\hline Malta \\
\hline Netherlands \\
\hline Poland \\
\hline Portugal \\
\hline Romania \\
\hline Slovak Republic \\
\hline Slovenia \\
\hline Spain \\
\hline Sweden \\
\hline European Central Bank \\
\hline EU Institutions \\
\hline Total EU27 \\
\hline European Free Trade Association (EFTA) \\
\hline Iceland \\
\hline Liechtenstein \\
\hline Norway \\
\hline Switzerland \\
\hline Total EFTA \\
\hline Other Europe \\
\hline Albania \\
\hline Belarus \\
\hline Croatia \\
\hline Russia \\
\hline Turkey \\
\hline Ukraine \\
\hline Serbia and Montenegro ${ }^{2}$ \\
\hline of which Serbia \\
\hline Montenegro \\
\hline $\begin{array}{c}\text { Other } \\
\text { Totarne }\end{array}$ \\
\hline Total Europe \\
\hline Americas \\
\hline Argentina \\
\hline Brazil \\
\hline Canada \\
\hline Chile \\
\hline Colombia \\
\hline Mexico \\
\hline United States of America \\
\hline Uruguay \\
\hline Venezuela \\
\hline Other Central American Countries \\
\hline Other \\
\hline Total Americas \\
\hline Asia \\
\hline China \\
\hline Hong Kong \\
\hline India \\
\hline Indonesia \\
\hline Iran \\
\hline Israel \\
\hline Japan \\
\hline Malaysia \\
\hline Pakistan \\
\hline Philippines \\
\hline Saudi Arabia \\
\hline Singapore \\
\hline South Korea \\
\hline Taiwan \\
\hline Thailand \\
\hline Residual Gulf Arabian Countries \\
\hline Other Near \& Middle Eastern Countries \\
\hline 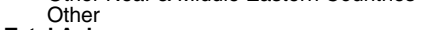 \\
\hline Total Asia \\
\hline Australasia \& Oceania \\
\hline Australia \\
\hline New Zealand \\
\hline Other \\
\hline Total Australasia \& Oceania \\
\hline Africa \\
\hline Egypt \\
\hline Morocco \\
\hline South Africa \\
\hline Other North Africa \\
\hline Other \\
\hline Total Africa \\
\hline International Organisations \\
\hline World total \\
\hline
\end{tabular}




\begin{tabular}{|c|}
\hline continued \\
\hline \\
\hline Debits \\
\hline $\begin{array}{l}\text { Europe } \\
\text { European Union (EU) }\end{array}$ \\
\hline Austria \\
\hline Belgium ${ }^{1}$ \\
\hline Bulgaria \\
\hline Cyprus \\
\hline Czech Republic \\
\hline Denmark \\
\hline Estonia \\
\hline Finland \\
\hline France \\
\hline Germany \\
\hline Greece \\
\hline Hungary \\
\hline Ireland \\
\hline Italy \\
\hline Latvia \\
\hline Lithuania \\
\hline Luxembourg $^{1}$ \\
\hline Malta \\
\hline Netherlands \\
\hline Poland \\
\hline Portugal \\
\hline Romania \\
\hline Slovak Republic \\
\hline Slovenia \\
\hline Spain \\
\hline Sweden \\
\hline European Central Bank \\
\hline EU Institutions \\
\hline Total EU27 \\
\hline European Free Trade Association (EFTA) \\
\hline Iceland \\
\hline Liechtenstein \\
\hline Norway \\
\hline Switzerland \\
\hline Total EFTA \\
\hline Other Europe \\
\hline Albania \\
\hline Belarus \\
\hline Croatia \\
\hline Russia \\
\hline Turkey \\
\hline Ukraine \\
\hline Serbia and Montenegro ${ }^{2}$ \\
\hline of which Serbia \\
\hline Montenegro \\
\hline Other \\
\hline Total Europe \\
\hline Americas \\
\hline Argentina \\
\hline Brazil \\
\hline Canada \\
\hline Chile \\
\hline Colombia \\
\hline Mexico \\
\hline United States of America \\
\hline Uruguay \\
\hline Venezuela \\
\hline Other Central American Countries \\
\hline Other \\
\hline Total Americas \\
\hline Asia \\
\hline China \\
\hline Hong Kong \\
\hline India \\
\hline Indonesia \\
\hline Iran \\
\hline Israel \\
\hline Japan \\
\hline Malaysia \\
\hline Pakistan \\
\hline Philippines \\
\hline Saudi Arabia \\
\hline Singapore \\
\hline South Korea \\
\hline Taiwan \\
\hline Thailand \\
\hline Residual Gulf Arabian Countries \\
\hline Other Near \& Middle Eastern Countries \\
\hline Other \\
\hline Total Asia \\
\hline Australasia \& Oceania \\
\hline Australia \\
\hline New Zealand \\
\hline Other \\
\hline Total Australasia \& Oceania \\
\hline Africa \\
\hline Egypt \\
\hline Morocco \\
\hline South Africa \\
\hline Other North Africa \\
\hline Other \\
\hline Total Africa \\
\hline International Organisations \\
\hline World total \\
\hline
\end{tabular}

1 Before 1999 only combined BLEU data is available under indentifier GXXW. 2 Separate Serbia and Montenegro data is available from 2007. 


\section{Current transfers}

continued

$2000 \quad 2001 \quad 2002 \quad 2003$

$£$ million

Balances

Europe

Auropean Union (EU)

Austria
Belgium

Belgium

Cyprus

Czech Republic

Denmark

Finland

France

Germany

Greece

Hungary

Italy

Lithuania

Luxembourg ${ }^{1}$

Malta

Poland

Portuga

Romania

Slovak Republic

Slovenia

Sweden

European Central Bank

EU Institutions

European Free Trade Association (EFTA)

celand

Liechtenstein

Norway

Switzerland
Total EFTA

Other Europe

Albania

Belarus

Russia

Turkey

Ukraine

Serbia and Montenegro ${ }^{2}$

of which Serbia

Other

Total Europe

Americas

Argentina

Brazil

Canada

Colombia

Mexico

United States of America

Uruguay

Other Central American Countries

Total Americas

Asia

China

Hong Kong

India

Indon

rrae

Japan

Malaysia
Pakistan

Philippines

Saudi Arabia

Singapore

South Korea

Taiwan

Thalf Arabian Countries Other Near \& Middle Eastern Countries Other Near

Total Asia

Australasia \& Oceania

New Zealan

Total Australasia \& Oceania

Africa

Egypt

South Africa

Other North Africa

Other

International Organisations

World total

\begin{tabular}{|c|c|c|c|c|c|c|c|c|c|c|c|}
\hline GZDU & 7 & 10 & 9 & 26 & 7 & 4 & 6 & 3 & -2 & 1 & -4 \\
\hline A8H2 & 139 & 120 & 143 & 123 & 126 & 163 & 172 & 148 & 133 & 132 & 34 \\
\hline ZWRH & -10 & -8 & -6 & -6 & -3 & 4 & - & 10 & 11 & 4 & \\
\hline A8H3 & -16 & -17 & -24 & -22 & -28 & -31 & -30 & -27 & -26 & -30 & -40 \\
\hline GZCJ & -3 & -7 & -8 & 20 & 10 & 2 & 18 & 1 & -3 & -4 & -8 \\
\hline GZDW & -9 & -2 & 5 & -2 & -2 & -9 & -1 & -6 & -11 & -1 & -10 \\
\hline ZWRN & - & - & - & -1 & -4 & -4 & -4 & -4 & - & -1 & \\
\hline GZDX & 2 & 1 & 2 & 80 & - & - & -1 & 3 & 2 & 3 & \\
\hline GZDY & 11 & 40 & 3 & -74 & 12 & -32 & -51 & -64 & -94 & -77 & 5 \\
\hline GZDZ & 97 & 104 & 78 & 63 & 56 & 44 & 72 & 63 & 24 & -164 & -278 \\
\hline GZEA & -10 & -5 & 2 & -6 & -10 & -10 & -9 & -20 & -28 & -22 & \\
\hline GYWH & -15 & -11 & -11 & -9 & -10 & -7 & -9 & - & -1 & -7 & \\
\hline GZEB & -356 & -280 & -321 & -393 & -389 & -445 & -428 & -427 & -468 & -480 & \\
\hline GZEC & -32 & -22 & -27 & -31 & - & -27 & -14 & -31 & -41 & -49 & \\
\hline ZWRV & - & - & 3 & - & - & 7 & -3 & 3 & 3 & 7 & \\
\hline zWRU & -1 & -14 & -14 & 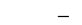 & -8 & -2 & 9 & 10 & 10 & 9 & \\
\hline A8H5 & 7 & 4 & 6 & 4 & 6 & 7 & 7 & 7 & 6 & 7 & \\
\hline A8H6 & -15 & -16 & -17 & -18 & -20 & -21 & -22 & -21 & -24 & -29 & \\
\hline GZED & 136 & 171 & 196 & 165 & 177 & 172 & 204 & 150 & 98 & 201 & 1 \\
\hline $\mathrm{GZCK}$ & -55 & -43 & -36 & -45 & -19 & 48 & 63 & 62 & 60 & -42 & \\
\hline GZEE & -20 & -21 & -19 & -26 & -24 & -27 & -30 & -28 & -33 & -25 & \\
\hline ZWRX & -14 & -11 & -9 & -9 & -10 & -5 & -9 & -4 & 3 & -1 & -1 \\
\hline ZWRZ & -5 & -1 & -3 & -3 & -1 & 7 & 61 & 16 & 17 & 8 & \\
\hline ZWRY & - & -5 & 1 & & - & - & - & (c) & - & 2 & \\
\hline GZEF & -33 & -28 & -36 & -65 & -44 & -35 & -25 & -62 & -78 & -85 & \\
\hline GYRO & 55 & 56 & 58 & 49 & 65 & 66 & 65 & 59 & 52 & 37 & \\
\hline ZWRB & & & & & & & & 507 & & & \\
\hline GYRP & -5405 & -2282 & -4192 & -4797 & -4151 & -5122 & -4906 & -5673 & -4783 & -5341 & -9 \\
\hline GD6K & -5545 & -2267 & -4217 & -4977 & -4264 & -5253 & -4865 & -5832 & -5173 & -5947 & \\
\hline GXEL & 29 & 32 & 29 & 62 & 32 & 33 & 33 & 32 & 31 & 30 & \\
\hline GXEM & & & & & & & & & & & \\
\hline GXEN & 4 & 1 & 17 & 12 & 3 & 10 & 14 & -11 & -18 & -13 & \\
\hline $\mathrm{GZCH}$ & -35 & -26 & -44 & $\begin{array}{l}-27 \\
\end{array}$ & -36 & -41 & -32 & -36 & -49 & -53 & \\
\hline $\mathrm{GZCI}$ & -2 & 7 & 2 & 47 & -1 & 2 & 15 & -15 & -36 & -36 & \\
\hline ZWRF & -6 & -9 & -12 & -7 & -7 & -7 & -4 & -9 & -9 & -10 & \\
\hline ZWRI & , & -5 & -11 & 4 & -2 & -1 & - & - & - & - & \\
\hline zWRS & -6 & -4 & -4 & -3 & -4 & -11 & -7 & -5 & -3 & -5 & \\
\hline GZCL & -80 & -43 & -7 & -38 & -41 & -38 & -50 & -38 & -11 & -54 & \\
\hline $\mathrm{GZCM}$ & - & -4 & -7 & -2 & -7 & -9 & -8 & -2 & -6 & -7 & \\
\hline zWSA & -29 & -54 & -15 & -21 & -22 & -40 & -20 & -18 & -16 & -24 & \\
\hline ZWSD & -65 & -37 & -29 & -25 & -26 & -29 & -28 & -29 & -32 & -39 & \\
\hline $\mathrm{KN} 4 \mathrm{P}$ & .. & .. & .. & .. & .. & .. & .. & -26 & -28 & -35 & \\
\hline KN4O & & & & & & & & -3 & -4 & -4 & \\
\hline ZWRC & $-11 \ddot{6}$ & -122 & -140 & -173 & $-15 \ddot{9}$ & $-16 \ddot{6}$ & -165 & -184 & -196 & -211 & \\
\hline
\end{tabular}

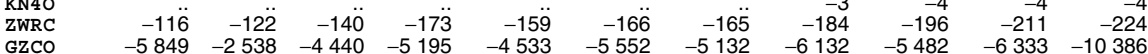

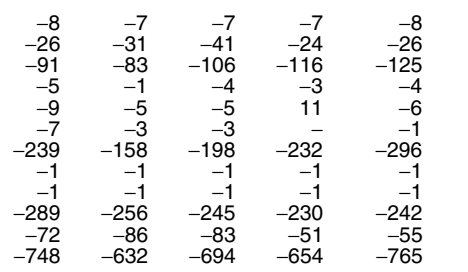

-10
-29
-139
-4
-6
-1
-285
-1
-1
-252
-53

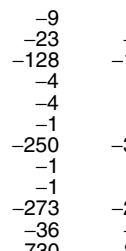

-9
-20
-155
-4
-6
-3
-313
-1
-1
-282
-48

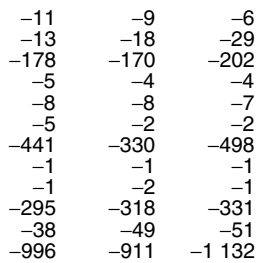

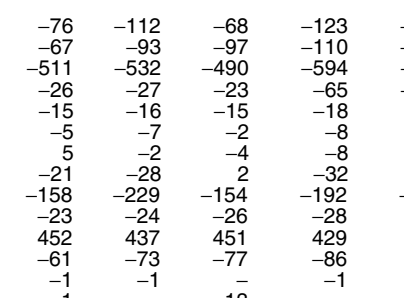

$\begin{array}{ll}-125 & -117 \\ -119 & -128 \\ -681 & -659\end{array}$

$-164 \quad-84 \quad-84$

$-9$

-11
-40
-208

-208
-30
441

-9
-4
-238
-3
444

444
-89

$-3$

-129
217

$-893$

$-1809$

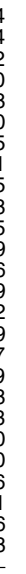




\section{Current account}

I. Transactions with Europe and USA ${ }^{1,2}$

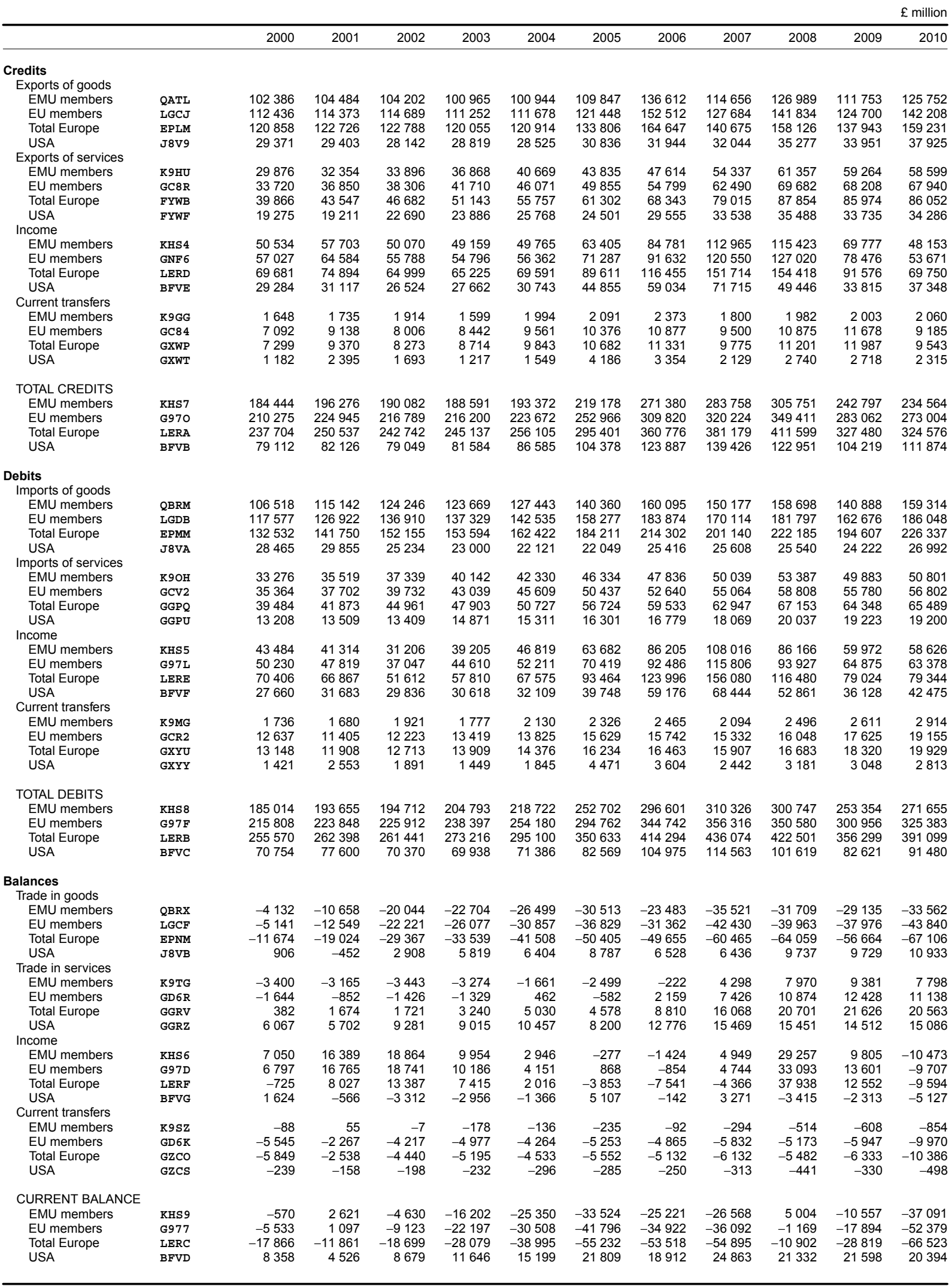

1 EMU Members: Austria, Belgium, Cyprus, Estonia, Finland, France, Germany,

Greece, Irish Republic, Italy, Luxembourg, Malta, Netherlands, Portugal, Slo-

vakia, Slovenia and Spain.
$2 \mathrm{EU}$ and Europe include transactions with European Union institutions. 


\section{(2) UK official transactions with institutions of the EU}

\begin{tabular}{|c|c|c|c|c|c|c|c|c|c|c|c|c|}
\hline & & & & & & & & & & & & $£$ million \\
\hline & & 2000 & 2001 & 2002 & 2003 & 2004 & 2005 & 2006 & 2007 & 2008 & 2009 & 2010 \\
\hline Credits & & & & & & & & & & & & \\
\hline Exports of services & & & & & & & & & & & & \\
\hline UK charge for collecting duties and levies(net) ${ }^{1}$ & QWUE & 217 & 525 & 487 & 489 & 543 & 565 & 583 & 603 & 660 & 661 & 733 \\
\hline Current transfers & & & & & & & & & & & & \\
\hline Other sectors & & & & & & & & & & & & \\
\hline Agricultural Guarantee Fund ${ }^{2}$ & EBGL & 2571 & 2336 & 2381 & 2691 & 3315 & 3408 & 3221 & 2952 & 3051 & 3411 & 3032 \\
\hline European Social Fund & HDIZ & 659 & 370 & 412 & 427 & 433 & 900 & 1305 & 795 & 608 & 609 & 777 \\
\hline European Coal \& Steel Community Grant & FUKP & - & 1 & - & - & 2 & - & - & - & - & - & - \\
\hline Central government & & & & & & & & & & & & \\
\hline Fontainebleau abatement & FKKL & 2084 & 4560 & 3099 & 3560 & 3592 & 3655 & 3570 & 3523 & 4862 & 5392 & 3046 \\
\hline Other EU receipts & GCSD & - & 8 & 13 & 10 & 12 & 13 & 24 & 117 & 30 & 26 & 15 \\
\hline Capital transfers & & & & & & & & & & & & \\
\hline Other sectors & & & & & & & & & & & & \\
\hline Agricultural Fund for Regional Development ${ }^{3}$ & FUXL & 82 & 26 & - & 2 & 49 & 80 & 50 & 150 & 417 & 215 & 431 \\
\hline European Regional Development Fund & HBZA & 989 & 543 & 296 & 622 & 1062 & 1402 & 618 & 707 & 972 & 640 & 609 \\
\hline Other capital transfers from EU Institutions ${ }^{2}$ & EBGO & - & 322 & - & - & - & - & - & - & - & - & - \\
\hline Total credits & GCSL & 6602 & 8691 & 6688 & 7801 & 9008 & 10023 & 9371 & 8847 & 10600 & 10954 & 8643 \\
\hline Debits & & & & & & & & & & & & \\
\hline Current transfers & & & & & & & & & & & & \\
\hline Othe & & & & & & & & & & & & \\
\hline Customs duties and agricultural levies ${ }^{4}$ & FUWD & 2086 & 2069 & 1919 & 1937 & 2145 & 2237 & 2329 & 2412 & 2636 & 2645 & 2933 \\
\hline Sugar levies ${ }^{4}$ & GTBA & 44 & 31 & 25 & 18 & 25 & 24 & - & - & - & - & - \\
\hline European Coal \& Steel Community production levy ${ }^{4}$ & GTBB & - & - & - & - & - & - & - & - & - & - & - \\
\hline VAT based contribution ${ }^{5}$ & HCML & 4104 & 3624 & 2720 & 2775 & 1764 & 1980 & 2165 & 2293 & 2255 & 1733 & 2172 \\
\hline VAT adjustment ${ }^{5}$ & FSVL & 100 & -49 & 88 & -35 & 25 & 19 & 2 & 26 & 15 & -140 & 81 \\
\hline Centr & & & & & & & & & & & & \\
\hline GNP fourth resource ${ }^{6}$ & HCSO & 4243 & 3859 & 5259 & 6622 & 7565 & 8597 & 8358 & 7996 & 8628 & 10692 & 10718 \\
\hline GNP adjustments ${ }^{6}$ & HCSM & 136 & -1 & 76 & 150 & -16 & 135 & 163 & 327 & -205 & -137 & 101 \\
\hline Total GNP based fourth own resource contribution & NMFH & 4379 & 3858 & 5335 & 6772 & 7549 & 8732 & 8521 & 8323 & 8423 & 10555 & 10819 \\
\hline Inter-government agreements & HCBW & - & - & - & - & - & - & - & - & - & - & - \\
\hline EU non-budget (miscellaneous) & HRTM & - & - & - & - & - & - & - & - & - & - & - \\
\hline Other current transfers to EU institutions & GVEG & 6 & 24 & 10 & 18 & -3 & 106 & 8 & 6 & 5 & -14 & -18 \\
\hline Total debits & GCSM & 10719 & 9557 & 10097 & 11485 & 11505 & 13098 & 13025 & 13060 & 13334 & 14779 & 15987 \\
\hline Balance (UK net contribution to the EU) & BLZS & -4117 & -866 & -3409 & -3684 & -2497 & -3075 & -3654 & -4213 & -2734 & -3825 & -7344 \\
\hline
\end{tabular}

1 Before 1989 this is netted off the VAT contribution but cannot be identfified separately.

Other capital transfers from EU institutions are included indistiguishably with Agricultural Guarantee Fund receipts before 1996.

3 Up to 2006 this series includes the European Agricultural Guidance Fund.

4 EU traditional own resource.

5 Third own resource contribution.

6 Fourth own resource contribution. 


\subsection{Tratel inenives \\ By type of service 2009}

\begin{tabular}{|c|c|c|c|c|c|c|c|c|c|c|c|c|}
\hline & $\begin{array}{c}\text { Trans- } \\
\text { port- } \\
\text { ation }\end{array}$ & Travel & $\begin{array}{r}\text { Commun- } \\
\text { ications }\end{array}$ & $\begin{array}{l}\text { Cons- } \\
\text { truction }\end{array}$ & $\begin{array}{l}\text { Insu- } \\
\text { rance }\end{array}$ & $\begin{array}{r}\text { Finan- } \\
\text { cial }\end{array}$ & $\begin{array}{r}\text { Computer } \\
\text { and } \\
\text { infor- } \\
\text { mation }\end{array}$ & $\begin{array}{r}\text { Royal- } \\
\text { ties } \\
\text { and } \\
\text { license } \\
\text { fees }\end{array}$ & $\begin{array}{r}\text { Other } \\
\text { busi- } \\
\text { ness } \\
\text { services }\end{array}$ & $\begin{array}{r}\text { Personal, } \\
\text { cultural } \\
\text { and } \\
\text { recrea- } \\
\text { tional }\end{array}$ & $\begin{array}{r}\text { Govern- } \\
\text { ment }\end{array}$ & $\begin{array}{r}\text { Total } \\
\text { services }\end{array}$ \\
\hline \multicolumn{13}{|l|}{ Exports } \\
\hline European Union (EU27) total & 8206 & 9010 & 2632 & 406 & 725 & 19167 & 3850 & 3252 & 19031 & 1050 & 879 & 68208 \\
\hline Belgium & 366 & 244 & 69 & .. & 24 & 983 & 170 & 59 & 1310 & 19 & .. & 3280 \\
\hline Luxembourg & 7 & 19 & 48 & - & 10 & 1451 & 58 & 8 & 761 & 1 & - & 2363 \\
\hline Denmark & 1126 & 286 & 44 & 6 & 32 & 394 & 99 & 133 & 744 & 16 & 7 & 2887 \\
\hline France & 927 & 1094 & 298 & 35 & 139 & 3271 & 438 & 346 & 2120 & 60 & 23 & 8751 \\
\hline Germany & 872 & 1107 & 515 & 31 & 114 & 3867 & 959 & 376 & 3420 & 183 & 32 & 11476 \\
\hline Ireland & 712 & 983 & 266 & 265 & 74 & 1603 & 967 & 965 & 2523 & 62 & 12 & 8432 \\
\hline Italy & 605 & 1420 & 340 & 22 & 118 & 1059 & 155 & 246 & 807 & 206 & 18 & 4996 \\
\hline Netherlands & 925 & 671 & 343 & 10 & 69 & 2960 & 339 & 240 & 3473 & 184 & 12 & 9226 \\
\hline Russia & 127 & 164 & 42 & 13 & 22 & 919 & 49 & 43 & 421 & 21 & 10 & 1831 \\
\hline Spain & 1614 & 945 & 304 & 8 & 40 & 1508 & 181 & 220 & 749 & 97 & 15 & 5681 \\
\hline Switzerland & 338 & 360 & 93 & 9 & 67 & 1921 & 1195 & 756 & 3085 & 68 & 3 & 7895 \\
\hline Turkey & 164 & 151 & 30 & 10 & 17 & 241 & 36 & 33 & 224 & 9 & 9 & 924 \\
\hline Argentina & 14 & 50 & 20 & & 11 & 12 & 4 & 7 & 22 & .. & .. & 146 \\
\hline Brazil & 110 & 200 & 46 & 12 & 22 & 101 & 29 & 15 & 152 & 1 & 6 & 694 \\
\hline Canada & 337 & 404 & 45 & 12 & 259 & 691 & 64 & 46 & 816 & 9 & 17 & 2700 \\
\hline Chile & 42 & 6 & 7 & 11 & 14 & 12 & & .. & 93 & .. & .. & 193 \\
\hline Mexico & 66 & 66 & 11 & .. & 36 & 92 & 12 & $2 \ddot{9}$ & 66 & &.. & 384 \\
\hline United States of America & 3121 & 2157 & 790 & & 3303 & 9626 & 2059 & 1751 & 9763 & 703 & & 33735 \\
\hline China & 737 & 600 & 48 & 13 & 17 & 204 & 34 & 43 & 436 & 85 & 13 & 2230 \\
\hline Hong Kong & 280 & 265 & 44 & 2 & 29 & 694 & 28 & 41 & 300 & 27 & 3 & 1713 \\
\hline India & 417 & 416 & 60 & 6 & 21 & 232 & 70 & 44 & 349 & 12 & 84 & 1711 \\
\hline Iran & 20 & 61 & 18 & 12 & 3 & 13 & 4 & & 63 & - & 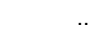 & 213 \\
\hline Japan & 525 & 376 & 46 & 2 & 108 & 2138 & 83 & 308 & 975 & 72 & $\ddot{4}$ & 4637 \\
\hline Malaysia & 41 & 191 & 19 & 3 & 17 & 88 & 32 & 35 & 230 & 15 & 3 & 674 \\
\hline Philippines & 34 & 39 & & & 12 & 12 & 11 & & 56 & & .. & 177 \\
\hline Saudi Arabia & 176 & 320 & 56 & $1 \ddot{4}$ & 18 & 303 & 26 & 10 & & $\ddot{7}$ &.. & 2410 \\
\hline Singapore & 592 & 123 & 39 & 5 & 28 & 501 & 48 & & 780 & 144 & & 4004 \\
\hline South Korea & 260 & 91 & 14 & 1 & 12 & 222 & 18 & 51 & 245 & 1 & $\ddot{6}$ & 921 \\
\hline Taiwan & 110 & 123 & 6 & 2 & 12 & 211 & .. & 24 & 67 & .. & 20 & 661 \\
\hline Thailand & 74 & 141 & 17 & 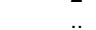 & 9 & 81 & 11 & 11 & 114 & & 6 & 497 \\
\hline Australia & 1321 & 819 & 76 & 79 & 157 & 706 & 110 & 93 & 748 & 57 & 13 & 4179 \\
\hline South Africa & 272 & 234 & 66 & 3 & 48 & 207 & 110 & 127 & 368 & 52 & 13 & 1500 \\
\hline Other & 3455 & 5227 & .. & .. & 779 & 10185 & .. & .. & .. &.. & .. & 36341 \\
\hline Global total & 19787 & 19353 & 5111 & 1561 & 5641 & 46508 & 8488 & 8829 & 47399 & 2601 & 2184 & 167462 \\
\hline
\end{tabular}

Imports

\begin{tabular}{|c|c|c|c|c|c|c|c|c|c|c|c|c|}
\hline European Union (EU27) total & 10793 & 18656 & 2039 & 518 & 519 & 3857 & 1962 & 1210 & 13122 & 510 & 2594 & 55780 \\
\hline Belgium & 396 & 364 & 66 & 8 & 42 & 452 & 57 & 161 & 829 & 6 & 25 & 2406 \\
\hline Luxembourg & 45 & 16 & 98 & & .. & 142 & 25 & 61 & 148 & & .. & 541 \\
\hline Denmark & 578 & 137 & 28 & 127 & & 82 & 27 & 68 & 283 & 76 & & 1429 \\
\hline France & 1470 & 3823 & 240 & 33 & 99 & 579 & 244 & 153 & 2477 & 56 & 110 & 9284 \\
\hline Germany & 930 & 753 & 691 & 120 & 111 & 819 & 518 & 212 & 2711 & 86 & 1983 & 8934 \\
\hline Ireland & 597 & 1007 & 216 & 117 & 38 & 535 & 402 & 122 & 1129 & 65 & 27 & 4255 \\
\hline Italy & 1254 & 1457 & 221 & 46 & 33 & 274 & 68 & 29 & 968 & 60 & 49 & 4459 \\
\hline Netherlands & 642 & 579 & 152 & 19 & 61 & 404 & 172 & 282 & 1861 & 25 & 27 & 4224 \\
\hline Russia & 158 & 118 & 28 & & 12 & 290 & 10 & 2 & 631 & & 11 & 1286 \\
\hline Spain & 2317 & 5668 & 125 & 10 & 26 & 232 & 58 & 25 & 795 & 21 & 159 & 9436 \\
\hline Switzerland & 422 & 527 & 42 & 11 & 16 & 382 & .. & 318 & 833 & 33 & .. & 2943 \\
\hline Turkey & 347 & 816 & 22 & 14 & 12 & 19 & - & 4 & 70 & .. & .. & 1320 \\
\hline Argentina & 15 & 48 & & .. & .. & 2 & .. & 2 & 22 & .. & 3 & 100 \\
\hline Brazil & 59 & 89 & 7 & 8 & 7 & & 5 & 6 & 82 & 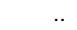 & 12 & 323 \\
\hline Canada & 190 & 380 & 63 & 12 & 14 & 120 & 50 & 88 & 296 & 5 & 61 & 1279 \\
\hline Chile & 42 & 15 & & .. &. & - & .. & .. & 9 & .. & .. & 73 \\
\hline Mexico & 45 & 170 & 5 & - & 2 & 9 & & 6 & 18 & & 4 & 265 \\
\hline United States of America & 1524 & 3128 & 439 & 102 & 109 & 3461 & 912 & 2810 & 5635 & 286 & 817 & 19223 \\
\hline China & 359 & 248 & 44 & 12 & 65 & 33 & 24 & 4 & 375 & & & 1177 \\
\hline Hong Kong & 146 & 130 & 36 & - & 20 & 307 & 10 & 2 & 316 & 3 & 4 & 974 \\
\hline India & 291 & 672 & 83 & 1 & 12 & 49 & 433 & 3 & 351 & 41 & 6 & 1942 \\
\hline Iran & 14 & 35 & & .. & & & & & 16 & & .. & 74 \\
\hline Japan & 300 & 129 & 31 & .. & 18 & 883 & 24 & 676 & 1203 & 25 & .. & 3306 \\
\hline Malaysia & 47 & 111 & 13 & .. & .. & 11 & 19 & 4 & 40 & .. & .. & 252 \\
\hline Philippines & 36 & 94 & & .. & .. & & 11 & .. & 26 & .. & .. & 181 \\
\hline Saudi Arabia & 34 & 95 & 11 & .. & .. & 13 & 2 & & & & 3 & 295 \\
\hline Singapore & 134 & 144 & 22 & .. & 9 & 205 & & 12 & 922 & 12 & .. & 1547 \\
\hline South Korea & 51 & 29 & 8 & .. & 8 & 76 & 10 & .. & 176 & .. & . & 364 \\
\hline Taiwan & 46 & 16 & 6 & .. & 6 & & & 1 & 107 & .. & 2 & 270 \\
\hline Thailand & 84 & 472 & 13 & - & $\cdot$. & 12 & 7 & & 31 & & .. & 630 \\
\hline Australia & 336 & 721 & 57 & .. & 8 & 109 & 50 & 63 & 315 & 11 & .. & 1739 \\
\hline South Africa & 141 & 400 & 31 & .. & 14 & 37 & 18 & .. & 204 & 25 & .. & 883 \\
\hline Other & 4979 & 9797 & .. & .. & .. & .. & .. & .. & .. & .. & .. & 24833 \\
\hline lobal total & 18029 & 32188 & 4402 & 1346 & 1007 & 10958 & 4002 & 5374 & 27742 & 1133 & 4066 & 110247 \\
\hline
\end{tabular}

Symbols used in this table:

Indicates that data might be disclosive and have therefore been omitted

- Indicates that the data is nil or less than $£ 500,000$ 


\subsection{Tratel insentices \\ By type of service 2010}

\begin{tabular}{|c|c|c|c|c|c|c|c|c|c|c|c|c|}
\hline & $\begin{array}{r}\text { Trans- } \\
\text { port- } \\
\text { ation }\end{array}$ & Travel & $\begin{array}{r}\text { Commun- } \\
\text { ications }\end{array}$ & $\begin{array}{l}\text { Cons- } \\
\text { truction }\end{array}$ & $\begin{array}{l}\text { Insu- } \\
\text { rance }\end{array}$ & $\begin{array}{r}\text { Finan- } \\
\text { cial }\end{array}$ & $\begin{array}{r}\text { Computer } \\
\text { and } \\
\text { infor- } \\
\text { mation }\end{array}$ & $\begin{array}{r}\text { Royal- } \\
\text { ties } \\
\text { and } \\
\text { license } \\
\text { fees }\end{array}$ & $\begin{array}{r}\text { Other } \\
\text { busi- } \\
\text { ness } \\
\text { services }\end{array}$ & $\begin{array}{r}\text { Personal, } \\
\text { cultural } \\
\text { and } \\
\text { recrea- } \\
\text { tional }\end{array}$ & $\begin{array}{r}\text { Govern- } \\
\text { ment }\end{array}$ & $\begin{array}{r}\text { Total } \\
\text { services }\end{array}$ \\
\hline \multicolumn{13}{|l|}{ Exports } \\
\hline European Union (EU27) total & 8118 & 9645 & 2384 & 331 & 750 & 17010 & 4068 & 3396 & 19575 & 1745 & 918 & 67940 \\
\hline Belgium & 334 & 410 & 57 & 18 & 23 & 708 & 122 & 59 & 1123 & 21 & 13 & 2888 \\
\hline Luxembourg & 6 & 24 & 93 & . & 17 & 1464 & 41 & & 472 & 15 & .. & 2144 \\
\hline Denmark & 1060 & 260 & 38 & 2 & 31 & 340 & 111 & 208 & 833 & 23 & 4 & 2910 \\
\hline France & 1029 & 1195 & 299 & 18 & 131 & 2801 & 442 & 264 & 2524 & 191 & 19 & 8913 \\
\hline Germany & 947 & 1252 & 394 & 25 & 111 & 3080 & 1097 & 402 & 3088 & 372 & 32 & 10800 \\
\hline Ireland & 805 & 913 & 287 & 232 & 49 & 1651 & 950 & 891 & 2498 & 105 & 8 & 8389 \\
\hline Italy & 601 & 1675 & 239 & 6 & 121 & 798 & 200 & 260 & 916 & 323 & 12 & 5151 \\
\hline Netherlands & 803 & 856 & 419 & 7 & 78 & 3286 & 355 & 272 & 3795 & 191 & 7 & 10069 \\
\hline Russia & 99 & 239 & 37 & 8 & 25 & 686 & 42 & 56 & 385 & 78 & 10 & 1665 \\
\hline Spain & 1509 & 853 & 209 & 8 & 54 & 1038 & 233 & 253 & 808 & 165 & 9 & 5139 \\
\hline Switzerland & 344 & 375 & 694 & 4 & 75 & 1793 & 1067 & 591 & 3980 & 55 & 3 & 8981 \\
\hline Turkey & 177 & 197 & 35 & 5 & 23 & 250 & 40 & 43 & 329 & 19 & 8 & 1126 \\
\hline Argentina & 29 & 59 & 14 & .. & 7 & 41 & 3 & 19 & & 5 & 4 & 233 \\
\hline Brazil & 116 & 174 & 48 & 4 & 40 & 139 & 67 & 18 & 221 & & & 836 \\
\hline Canada & 379 & 562 & 126 & 17 & 382 & 638 & 91 & 40 & 647 & 9 & 14 & 2905 \\
\hline Chile & 63 & 16 & 10 & 9 & 20 & 8 & & & 46 & .. & .. & 181 \\
\hline Mexico & 73 & 50 & 12 & - & 51 & 114 & 15 & 32 & 66 & . & .. & 421 \\
\hline United States of America & 3584 & 2354 & 658 & 39 & 2497 & 9115 & 2020 & 1755 & 11029 & 936 & 299 & 34286 \\
\hline China & 729 & 813 & 51 & 7 & 27 & 218 & 68 & 47 & 609 & 69 & 14 & 2652 \\
\hline Hong Kong & 253 & 240 & 42 & .. & 30 & 598 & 48 & 28 & 345 & .. & & 1606 \\
\hline India & 436 & 582 & 42 & 3 & 27 & 216 & 67 & 26 & 569 & 10 & 82 & 2060 \\
\hline Iran & 13 & 42 & 9 & - & 2 & 12 & 4 & 5 & 25 & .. &.. & 128 \\
\hline Japan & 495 & 213 & .. & .. & 109 & 1811 & 96 & 280 & 1166 & 101 & .. & 4315 \\
\hline Malaysia & 39 & 185 & 15 & 4 & 24 & 95 & 24 & 23 & 231 & .. & .. & 701 \\
\hline Philippines & 34 & 16 & 5 & - & 5 & 10 & 8 & 4 & 42 & - & 1 & 125 \\
\hline Saudi Arabia & 118 & 290 & 43 & 51 & 19 & 282 & 38 & 22 & & 35 & .. & 2925 \\
\hline Singapore & 497 & 194 & 28 & 4 & 43 & 449 & 62 & & 785 & 96 & .. & 3661 \\
\hline South Korea & 281 & 116 & 13 & .. & 26 & 208 & 30 & 42 & 396 & .. & .. & 1123 \\
\hline Taiwan & 130 & 112 & 6 & .. & 7 & 556 & 83 & 29 & 200 & .. & .. & 1145 \\
\hline Thailand & 58 & 154 & 18 & - & 11 & 75 & 16 & 6 & 100 & & .. & 459 \\
\hline Australia & 1732 & 982 & 75 & 120 & 295 & 719 & 80 & 89 & 810 & 111 & 12 & 5025 \\
\hline South Africa & 285 & 219 & 60 & 2 & 63 & 243 & 151 & 157 & 431 & 98 & 14 & 1723 \\
\hline Other & 3271 & 5347 & .. &.. & 842 & 8720 & .. &.. &.. & .. &.. & 36397 \\
\hline Global total & 20329 & 20969 & 5340 & 1218 & 5265 & 42162 & 8742 & 8678 & 52162 & 4012 & 2205 & 171082 \\
\hline
\end{tabular}

Imports

\begin{tabular}{|c|c|c|c|c|c|c|c|c|c|c|c|c|}
\hline European Union (EU27) total & 10764 & 18035 & 2504 & 551 & 625 & 3807 & 1915 & 1232 & 13798 & 870 & 2701 & 56802 \\
\hline Belgium & 413 & 330 & 65 & 13 & 49 & 413 & 66 & 109 & 844 & 6 & 11 & 2319 \\
\hline Luxembourg & 21 & 29 & 770 & & & 248 & & 24 & 198 & & 1 & 1411 \\
\hline Denmark & 604 & 99 & 26 & 17 & 15 & 87 & 35 & 77 & 294 & 31 & 9 & 1294 \\
\hline France & 1424 & 3789 & 186 & 61 & 95 & 574 & 221 & 145 & 2834 & 126 & 181 & 9636 \\
\hline Germany & 905 & 797 & 640 & 212 & 139 & 867 & 485 & 228 & 2916 & 99 & 1839 & 9127 \\
\hline Ireland & 578 & 862 & 199 & 87 & 58 & 448 & 488 & 198 & 1145 & 47 & 40 & 4150 \\
\hline Italy & 1167 & 1345 & 192 & 59 & 42 & 153 & 43 & 18 & 999 & 268 & 64 & 4350 \\
\hline Netherlands & 636 & 587 & 151 & 23 & 79 & 528 & 143 & 278 & 1352 & 87 & 34 & 3898 \\
\hline Russia & 154 & 86 & 24 & & 17 & 89 & 13 & 3 & 493 & 10 & .. & 903 \\
\hline Spain & 2334 & 5425 & 91 & 13 & 31 & 232 & 75 & 27 & 930 & 68 & 256 & 9482 \\
\hline Switzerland & 381 & 546 & 184 & & 25 & 453 & 220 & 326 & 1034 & 33 & .. & 3215 \\
\hline Turkey & 438 & 1016 & 9 & 8 & 16 & 36 &.. & 4 & 79 & 5 & .. & 1623 \\
\hline Argentina & 18 & 49 & 4 & .. & .. & 2 & .. & .. & 24 & 5 & 2 & 111 \\
\hline Brazil & 63 & 131 & 8 & - & 11 & & 5 & 3 & 107 & 21 & .. & 447 \\
\hline Canada & 199 & 412 & 140 & 17 & 41 & 133 & 32 & 80 & 361 & 6 & 56 & 1477 \\
\hline Chile & 9 & 14 & .. & .. & .. & . & .. & .. & 18 & .. & .. & 50 \\
\hline Mexico & 72 & 254 & 5 & - & .. & 14 & .. & 1 & 29 & 2 & 3 & 398 \\
\hline United States of America & 1664 & 3455 & 409 & 72 & 322 & 3356 & 844 & 2416 & 5471 & 318 & 873 & 19200 \\
\hline China & 362 & 281 & 44 & 14 & 78 & 41 & 33 & 1 & 315 & 4 & 7 & 1180 \\
\hline Hong Kong & 134 & 170 & & & 26 & 358 & 13 & 1 & 301 & 5 & 2 & 1056 \\
\hline India & 405 & 702 & 56 & $\ddot{9}$ & 17 & 65 & 539 & 3 & 481 & 7 & 8 & 2292 \\
\hline Iran & 14 & 44 & .. & - & .. & - & &.. & .. & .. & 1 & 66 \\
\hline Japan & 295 & 114 & 34 & .. & 33 & 775 & 16 & 987 & 1339 & 19 & .. & 3627 \\
\hline Malaysia & 42 & 115 & 13 & .. & 7 & 14 & 9 & 1 & 57 & .. & .. & 260 \\
\hline Philippines & 49 & 93 & & .. & .. & & 16 & 1 & 50 & .. & .. & 217 \\
\hline Saudi Arabia & 39 & 84 & 10 & .. & .. & 16 & & .. & 53 & & 2 & 249 \\
\hline Singapore & 127 & 115 & 18 & - & 13 & 198 & 28 & .. & 1237 & 10 & .. & 1803 \\
\hline South Korea & 49 & 23 & 6 & .. & 10 & 88 & 12 & .. & 156 & .. & .. & 351 \\
\hline Taiwan & 37 & 27 & .. & - & 8 & 72 & .. & .. & 91 & .. & 1 & 247 \\
\hline Thailand & 92 & 458 & 13 & - & 8 & & 5 & 1 & 23 & 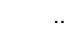 & 1 & 624 \\
\hline Australia & 476 & 800 & 57 & .. & 24 & 131 & 108 & 63 & 420 & 19 & .. & 2194 \\
\hline South Africa & 149 & 419 & 48 & $\ddot{1}$ & 20 & 55 & 27 & 8 & 358 & 13 & $\ddot{3}$ & 1101 \\
\hline Other & 5360 & 9689 & .. & .. &.. & .. & .. & .. & .. & .. & .. & 23946 \\
\hline lobal total & 18710 & 32360 & 4396 & 1229 & 1561 & 10667 & 3968 & 5281 & 28472 & 1511 & 4149 & 112304 \\
\hline
\end{tabular}

Symbols used in this table:

.. Indicates that data might be disclosive and have therefore been omitted

- Indicates that the data is nil or less than $£ 500,000$ 


\section{Trade in goods and services Top fifty UK trading partners}

\begin{tabular}{|c|c|c|c|c|c|c|c|c|c|c|c|}
\hline \multicolumn{6}{|c|}{ Exports } & \multicolumn{6}{|c|}{ Imports } \\
\hline \multicolumn{3}{|c|}{ Goods (£266 bn in 2010) } & \multicolumn{3}{|c|}{ Services (£171bn in 2010) } & \multicolumn{3}{|c|}{ Goods (£364bn in 2010) } & \multicolumn{3}{|c|}{ Services (£112bn in 2010) } \\
\hline & 10 & 09 & & 10 & 09 & & 10 & 09 & & 10 & 09 \\
\hline$U_{S A}{ }^{1}$ & 1 & 1 & $\mathrm{USA}^{1}$ & 1 & 1 & Germany & 1 & 1 & $U_{S A}^{1}$ & 1 & 1 \\
\hline Germany & 2 & 2 & Germany & 2 & 2 & China & 2 & 3 & France & 2 & 3 \\
\hline Netherlands & 3 & 3 & Netherlands & 3 & 3 & USA $^{1}$ & 3 & 2 & Spain & 3 & 2 \\
\hline France & 4 & 4 & Switzerland & 4 & 6 & Netherlands & 4 & 4 & Germany & 4 & 4 \\
\hline Irish Republic & 5 & 5 & France & 5 & 4 & France & 5 & 5 & Italy & 5 & 5 \\
\hline Belgium-Lux & 6 & 6 & Ireland & 6 & 5 & Norway & 6 & 6 & Ireland & 6 & 6 \\
\hline Spain & 7 & 7 & Italy & 7 & 8 & Belgium-Lux & 7 & 7 & Netherlands & 7 & 7 \\
\hline Italy & 8 & 8 & Spain & 8 & 7 & Italy & 8 & 9 & Japan & 8 & 8 \\
\hline China & 9 & 9 & Australia & 9 & 10 & Irish Republic & 9 & 8 & Switzerland & 9 & 9 \\
\hline Sweden & 10 & 10 & Japan & 10 & 9 & Spain & 10 & 10 & Belgium & 10 & 10 \\
\hline Switzerland & 11 & 11 & Singapore & 11 & 11 & Hong Kong & 11 & 11 & India & 11 & 12 \\
\hline Hong Kong & 12 & 12 & Saudi Arabia & 12 & 18 & Japan & 12 & 12 & Australia & 12 & 14 \\
\hline Japan & 13 & 14 & Sweden & 13 & 16 & Switzerland & 13 & 14 & Greece & 13 & 11 \\
\hline Canada & 14 & 15 & Denmark & 14 & 14 & Sweden & 14 & 13 & Singapore & 14 & 15 \\
\hline India & 15 & 18 & Canada & 15 & 15 & Poland & 15 & 15 & Portugal & 15 & 16 \\
\hline United Arab Emirates $^{2}$ & 16 & 13 & Belgium & 16 & 13 & India & 16 & 18 & Turkey & 16 & 19 \\
\hline Poland & 17 & 20 & The Channel Islands & 17 & 12 & Canada & 17 & 19 & Sweden & 17 & 13 \\
\hline Russia & 18 & 22 & China & 18 & 20 & Turkey & 18 & 17 & Canada & 18 & 22 \\
\hline Singapore & 19 & 16 & Norway & 19 & 17 & Russia & 19 & 16 & Luxembourg & 19 & 34 \\
\hline Australia & 20 & 17 & United Arab Emirates ${ }^{2}$ & 20 & 22 & South Africa & 20 & 21 & Poland & 20 & 23 \\
\hline Turkey & 21 & 23 & Luxembourg & 21 & 19 & Singapore & 21 & 22 & Denmark & 21 & 18 \\
\hline Norway & 22 & 19 & India & 22 & 25 & Denmark & 22 & 20 & China & 22 & 25 \\
\hline South Africa & 23 & 25 & South Africa & 23 & 26 & Czech Republic & 23 & 23 & United Arab Emirates ${ }^{2}$ & 23 & 20 \\
\hline Denmark & 24 & 21 & Russia & 24 & 23 & Hungary & 24 & 25 & South Africa & 24 & 30 \\
\hline Saudi Arabia & 25 & 24 & Cayman Islands & 25 & 21 & Taiwan & 25 & 29 & Norway & 25 & 24 \\
\hline South Korea & 26 & 26 & Hong Kong & 26 & 24 & Brazil & 26 & 26 & Bermuda & 26 & 17 \\
\hline Brazil & 27 & 27 & Nigeria & 27 & 27 & Thailand & 27 & 27 & The Channel Islands & 27 & 26 \\
\hline Portugal & 28 & 29 & Finland & 28 & 28 & Austria & 28 & 28 & Hong Kong & 28 & 27 \\
\hline Czech Rep & 29 & 30 & Taiwan & 29 & 40 & South Korea & 29 & 24 & Austria & 29 & 29 \\
\hline Finland & 30 & 31 & Poland & 30 & 29 & Qatar & 30 & 43 & Russia & 30 & 21 \\
\hline Austria & 31 & 33 & Turkey & 31 & 31 & Australia & 31 & 30 & Cyprus & 31 & 28 \\
\hline Nigeria & 32 & 32 & South Korea & 32 & 32 & Finland & 32 & 31 & Egypt & 32 & 31 \\
\hline Israel & 33 & 34 & Greece & 33 & 30 & Malaysia & 33 & 32 & Thailand & 33 & 32 \\
\hline Greece & 34 & 28 & Virgin Islands, British & 34 & 35 & Portugal & 34 & 34 & Hungary & 34 & 35 \\
\hline Malaysia & 35 & 35 & Brazil & 35 & 38 & United Arab Emirates ${ }^{2}$ & 35 & 37 & Finland & 35 & 41 \\
\hline Egypt & 36 & 36 & Angola & 36 & 48 & Slovakia & 36 & 33 & Brazil & 36 & 44 \\
\hline Thailand & 37 & 37 & Portugal & 37 & 36 & Israel & 37 & 39 & Pakistan & 37 & 37 \\
\hline Taiwan & 38 & 40 & Malaysia & 38 & 39 & Libya & 38 & 42 & Czech Republic & 38 & 39 \\
\hline Hungary & 39 & 38 & Bermuda & 39 & 33 & Indonesia & 39 & 35 & Israel & 39 & 42 \\
\hline Qatar & 40 & 39 & Israel & 40 & 43 & Vietnam & 40 & 36 & Afghanistan & 40 & - \\
\hline Mexico & 41 & 41 & Austria & 41 & 37 & Romania & 41 & 41 & Mexico & 41 & 50 \\
\hline Romania & 42 & 42 & Cyprus & 42 & 45 & Bangladesh & 42 & 38 & New Zealand & 42 & 45 \\
\hline Chile & 43 & 45 & Kazakhstan & 43 & 34 & Kuwait & 43 & 46 & South Korea & 43 & 40 \\
\hline Angola & 44 & - & Egypt & 44 & 41 & Mexico & 44 & 44 & Bulgaria & 44 & 49 \\
\hline Morocco & 45 & - & Qatar & 45 & 49 & Nigeria & 45 & 50 & Malta & 45 & 43 \\
\hline Cyprus & 46 & 43 & Kuwait & 46 & - & New Zealand & 46 & 40 & Barbados & 46 & 38 \\
\hline Kuwait & 47 & 47 & Hungary & 47 & 44 & Pakistan & 47 & 47 & Nigeria & 47 & 33 \\
\hline Senegal & 48 & - & Thailand & 48 & 47 & Saudi Arabia & 48 & - & Malaysia & 48 & - \\
\hline Ukraine & 49 & 44 & Czech Republic & 49 & 50 & Sri Lanka & 49 & 45 & Romania & 49 & - \\
\hline Slovakia & 50 & - & New Zealand & 50 & - & Algeria & 50 & - & Saudi Arabia & 50 & 45 \\
\hline
\end{tabular}

1 USA includes Puerto Rico

2 United Arab Emirates includes Abu Dhabi, Dubai, Sharjah, Ajman,

Umm al Qaiwain, Ras al Khaimah and Fujairah 


\subsection{Tratelin senvices \\ Trading partners ranked ${ }^{1}$}

\begin{tabular}{|c|c|c|c|c|c|c|c|c|c|}
\hline \multirow{2}{*}{ Exports } & & & & & & & & \multirow{2}{*}{\multicolumn{2}{|c|}{$£$ million }} \\
\hline & & & & & & & & & \\
\hline & 2010 & 2009 & & 2010 & 2009 & & 2010 & 200 & \\
\hline USA & 34286 & 33735 & Kenya & 200 & 178 & Belize & 17 & & 18 \\
\hline Germany & 10800 & 11476 & Bangladesh & 198 & 169 & Cuba & 17 & & 20 \\
\hline Netherlands & 10069 & 9226 & Viet Nam & 192 & 239 & Guinea & 17 & & 22 \\
\hline Switzerland & 8981 & 7895 & Bahamas & 186 & 164 & Somalia & 17 & & 5 \\
\hline France & 8913 & 8751 & Chile & 181 & 193 & Armenia & 16 & & 6 \\
\hline Ireland & 8389 & 8432 & Iceland & 168 & 147 & Bosnia \& Herzegovina & 16 & & 9 \\
\hline Italy & 5151 & 4996 & Bulgaria & 164 & 204 & Andorra & 15 & & 15 \\
\hline Spain & 5139 & 5681 & Oman & 164 & 148 & Macao & 14 & & 9 \\
\hline Australia & 5025 & 4179 & Isle of Man & 156 & 202 & Seychelles & 14 & & 33 \\
\hline Japan & 4315 & 4637 & Morocco & 137 & 167 & Macedonia & 13 & & 15 \\
\hline Singapore & 3661 & 4004 & Sri Lanka & 135 & 83 & Rwanda & 13 & & 7 \\
\hline Saudi Arabia & 2925 & 2410 & Algeria & 133 & 147 & Albania & 12 & & 13 \\
\hline Sweden & 2911 & 2623 & Iran & 128 & 213 & Mali & 12 & & 18 \\
\hline Denmark & 2910 & 2887 & Philippines & 125 & 177 & Mongolia & 12 & & 10 \\
\hline Canada & 2905 & 2700 & Lebanon & 124 & 106 & Uzbekistan & 12 & & 7 \\
\hline Belgium & 2888 & 3280 & Jordan & 123 & 131 & Cambodia & 11 & & 11 \\
\hline The Channel Islands & 2834 & 3334 & Trinidad \&Tobago & 116 & 147 & Montenegro & 11 & & 14 \\
\hline China & 2652 & 2230 & Panama & 115 & 67 & Turkmenistan & 11 & & 7 \\
\hline Norway & 2383 & 2424 & Tunisia & 115 & 165 & Aruba & 10 & & 12 \\
\hline United Arab Emirates2 & 2166 & 1916 & Cameroon & 113 & 108 & Belarus & 10 & & 4 \\
\hline Luxembourg & 2144 & 2363 & Peru & 106 & 124 & Democratic Republic Congo & 9 & & 11 \\
\hline India & 2060 & 1711 & Lithuania & 96 & 93 & Guatemala & 9 & & 8 \\
\hline South Africa & 1723 & 1500 & Slovenia & 92 & 84 & Maldives & 9 & & 11 \\
\hline Russia & 1665 & 1831 & Latvia & 87 & 110 & Micronesia, & 9 & & 4 \\
\hline Cayman Islands & 1643 & 1950 & Barbados & 84 & 98 & Moldova & 9 & & 10 \\
\hline Hong Kong & 1606 & 1713 & Zambia & 84 & 47 & Solomon Islands & 9 & & 6 \\
\hline Nigeria & 1280 & 1323 & Croatia & 82 & 105 & Togo & 9 & & 9 \\
\hline Finland & 1274 & 1304 & Mozambique & 80 & 86 & Benin & 8 & & 10 \\
\hline Taiwan & 1145 & 661 & Estonia & 79 & 43 & Cape Verde & 8 & & 9 \\
\hline Poland & 1132 & 1053 & Sudan & 78 & 58 & Mauritania & 8 & & 10 \\
\hline Turkey & 1126 & 924 & Papua New Guinea & 75 & 83 & Nicaragua & 8 & & 12 \\
\hline South Korea & 1123 & 921 & Congo & 72 & 62 & North Korea & 8 & & 15 \\
\hline Greece & 1119 & 1037 & Equatorial Guinea & 66 & 72 & Saint Lucia & 8 & & 4 \\
\hline Virgin Islands, British & 1115 & 799 & Senegal & 65 & 55 & America Samoa & 7 & & 2 \\
\hline Brazil & 836 & 694 & Netherlands Antilles & 63 & 65 & Guam & 7 & & 2 \\
\hline Angola & 781 & 496 & Uganda & 62 & 74 & Kyrgyzstan & 7 & & 18 \\
\hline Portugal & 779 & 771 & Liechtenstein & 60 & 72 & Turks \& Caicos Islands & 7 & & 13 \\
\hline Malaysia & 701 & 674 & Brunei Darussalam & 54 & 47 & Tajikistan & 7 & & 5 \\
\hline Bermuda & 695 & 913 & Georgia & 53 & 43 & Vanuatu & 7 & & 1 \\
\hline Israel & 682 & 585 & Marshall Islands & 53 & 17 & Burundi & 6 & & 1 \\
\hline Austria & 643 & 744 & Tanzania & 52 & 54 & Gambia & 6 & & 9 \\
\hline Cyprus & 602 & 551 & Gabon & 50 & 45 & Myanmar & 6 & & 4 \\
\hline Kazakhstan & 590 & 806 & Syrian Arab Republic & 50 & 65 & Tonga & 6 & & 5 \\
\hline Egypt & 560 & 657 & Serbia & 48 & 57 & Antigua \& Barbuda & 5 & & 4 \\
\hline Qatar & 504 & 489 & Botswana & 47 & 52 & Lesotho & 5 & & 8 \\
\hline Kuwait & 485 & 626 & Guyana & 45 & 36 & San Marino & 5 & & 1 \\
\hline Hungary & 472 & 552 & Ethiopia & 39 & 45 & Burkina Faso & 4 & & 7 \\
\hline Thailand & 459 & 497 & Cote d'Ivoire & 36 & 76 & Djibouti & 4 & & 1 \\
\hline Czech Republic & 452 & 466 & Ecuador & 35 & 21 & El Salvador & 4 & & 9 \\
\hline New Zealand & 452 & 426 & Dominican Republic & 34 & 49 & Haiti & 4 & & 4 \\
\hline Afghanistan & 432 & 244 & Malawi & 34 & 30 & Honduras & 4 & & 10 \\
\hline Mexico & 421 & 384 & Jamaica & 33 & 44 & Kiribati & 4 & & 3 \\
\hline Iraq & 413 & 346 & Yemen & 29 & 40 & Palau & 4 & & 1 \\
\hline Romania & 359 & 283 & Suriname & 28 & 27 & Swaziland & 4 & & 29 \\
\hline Venezuela & 354 & 75 & Zimbabwe & 28 & 40 & Comoros & 3 & & 1 \\
\hline Indonesia & 325 & 325 & Namibia & 27 & 27 & Eritrea & 3 & & 2 \\
\hline Pakistan & 302 & 357 & Fiji & 26 & 9 & Grenada & 3 & & 3 \\
\hline Slovakia & 291 & 242 & Nepal & 25 & 16 & St Vincent \& the Grenadines & 3 & & 4 \\
\hline Gibraltar & 273 & 516 & Liberia & 24 & 12 & Virgin Islands,U.S. & 3 & & 3 \\
\hline Libyan Arab Jamahiriya & 256 & 258 & Falkland Islands (Malvinas) & 23 & 15 & Guinea-Bissau & 2 & & 1 \\
\hline Bahrain & 246 & 302 & Sierra Leone & 23 & 48 & Democratic Republic Lao & 2 & & 3 \\
\hline Azerbaijan & 244 & 296 & Chad & 23 & 25 & St Kitts \& Nevis & 2 & & 4 \\
\hline Ukraine & 244 & 177 & Costa Rica & 22 & 30 & St Helena & 2 & & 8 \\
\hline Mauritius & 240 & 159 & Madagascar & 22 & 22 & Anguilla & 1 & & 1 \\
\hline Argentina & 233 & 146 & Bolivia & 21 & 24 & Bhutan & 1 & & 1 \\
\hline Malta & 227 & 283 & Niger & 21 & 5 & Holy see (Vatican City State) & 1 & & 1 \\
\hline Ghana & 203 & 331 & Uruguay & 20 & 15 & & & & \\
\hline Colombia & 200 & 92 & Paraguay & 19 & 14 & & & & \\
\hline
\end{tabular}

1 Any country not included in this table is estimated having

Trade in Services of less than $£ 500,000$

2 United Arab Emirates includes Abu Dhabi, Dubai, Sharjah, Ajman,

Umm al Qaiwain, Ras al Khaimah and Fujairah 
Trade in services

Trading partners ranked ${ }^{1}$

\begin{tabular}{|c|c|c|c|c|c|c|c|c|}
\hline Imports & & & & & & & & \\
\hline & 2010 & 2009 & & 2010 & 2009 & & 2010 & 2009 \\
\hline USA & 19200 & 19223 & Libyan Arab Jamahiriya & 129 & 110 & Belarus & 9 & 6 \\
\hline France & 9636 & 9284 & Bangladesh & 122 & 136 & Gabon & 9 & 3 \\
\hline Spain & 9482 & 9436 & Slovakia & 121 & 124 & Guyana & 9 & 8 \\
\hline Germany & 9127 & 8934 & Indonesia & 119 & 121 & Myanmar & 9 & 2 \\
\hline Italy & 4350 & 4459 & Maldives & 119 & 138 & Brunei Darussalam & 8 & 3 \\
\hline Ireland & 4150 & 4255 & Lithuania & 118 & 101 & Democratic Republic Congo & 8 & 8 \\
\hline Netherlands & 3898 & 4224 & Virgin Islands, British & 115 & 165 & Cote d`Ivoire & 8 & 10 \\
\hline Japan & 3627 & 3306 & Argentina & 111 & 100 & Democratic Republic Lao & 8 & 5 \\
\hline Switzerland & 3215 & 2943 & Viet Nam & 97 & 78 & Namibia & 8 & 15 \\
\hline Belgium & 2319 & 2406 & Latvia & 94 & 56 & Uruguay & 8 & 26 \\
\hline India & 2292 & 1942 & Bahrain & 93 & 67 & Eritrea & 7 & 6 \\
\hline Australia & 2194 & 1739 & Ghana & 90 & 95 & Marshall Islands & 7 & 1 \\
\hline Greece & 1951 & 2112 & Qatar & 89 & 167 & Mauritania & 7 & 1 \\
\hline Singapore & 1803 & 1547 & Azerbaijan & 85 & 117 & Senegal & 7 & 11 \\
\hline Portugal & 1633 & 1515 & Algeria & 70 & 69 & Madagascar & 6 & 11 \\
\hline Turkey & 1623 & 1320 & Dominican Republic & 69 & 120 & Turkmenistan & 6 & 2 \\
\hline Sweden & 1614 & 1758 & Tanzania & 69 & 63 & Anguilla & 5 & 1 \\
\hline Canada & 1477 & 1279 & Iran & 66 & 74 & Bosnia \& Herzegovina & 5 & 38 \\
\hline Luxembourg & 1411 & 541 & Peru & 64 & 67 & Mozambique & 5 & 16 \\
\hline Poland & 1361 & 1255 & Antigua \& Barbuda & 61 & 56 & Swaziland & 5 & 2 \\
\hline Denmark & 1294 & 1429 & Lebanon & 57 & 33 & Albania & 4 & 13 \\
\hline China & 1180 & 1177 & Iceland & 56 & 130 & Benin & 4 & 1 \\
\hline United Arab Emirates2 & 1150 & 1303 & Colombia & 55 & 51 & Bolivia & 4 & 11 \\
\hline South Africa & 1101 & 883 & Chile & 50 & 73 & Burkina Faso & 4 & 1 \\
\hline Norway & 1092 & 1199 & Venezuela & 50 & 42 & Falkland Islands & 4 & 4 \\
\hline Bermuda & 1069 & 1498 & Zimbabwe & 49 & 45 & Honduras & 4 & 1 \\
\hline The Channel Islands & 1059 & 1052 & Slovenia & 48 & 40 & Paraguay & 4 & 2 \\
\hline Hong Kong & 1056 & 974 & Oman & 46 & 47 & Belize & 3 & 12 \\
\hline Austria & 987 & 908 & Jordan & 44 & 46 & Cape Verde & 3 & 2 \\
\hline Russia & 903 & 1286 & Nepal & 43 & 35 & Mali & 3 & 4 \\
\hline Cyprus & 881 & 934 & Seychelles & 43 & 23 & Moldova & 3 & 10 \\
\hline Egypt & 681 & 741 & Zambia & 42 & 56 & North Korea & 3 & 6 \\
\hline Thailand & 624 & 630 & Cayman Islands & 41 & 36 & Armenia & 2 & 3 \\
\hline Hungary & 486 & 418 & Ecuador & 38 & 30 & America Samoa & 2 & - \\
\hline Finland & 451 & 354 & Serbia & 38 & 49 & Cambodia & 2 & 6 \\
\hline Brazil & 447 & 323 & Saint Lucia & 36 & 61 & Djibouti & 2 & 5 \\
\hline Pakistan & 443 & 389 & Isle of Man & 34 & 18 & Guam & 2 & \\
\hline Czech Republic & 434 & 372 & Estonia & 30 & 30 & Kyrgyzstan & 2 & 1 \\
\hline Israel & 415 & 349 & Guatemala & 30 & 22 & Liberia & 2 & 3 \\
\hline Afghanistan & 399 & 238 & Syrian Arab Republic & 30 & 20 & Netherlands Antilles & 2 & 4 \\
\hline Mexico & 398 & 265 & Trinidad \&Tobago & 30 & 48 & Rwanda & 2 & 5 \\
\hline New Zealand & 354 & 283 & Uganda & 28 & 53 & Antartica & 1 & - \\
\hline South Korea & 351 & 364 & Bahamas & 27 & 61 & Dominica & 1 & 2 \\
\hline Bulgaria & 340 & 265 & Grenada & 26 & 43 & Equatorial Guinea & 1 & 1 \\
\hline Malta & 326 & 327 & Costa Rica & 25 & 37 & Guinea & 1 & 3 \\
\hline Barbados & 311 & 373 & Andorra & 23 & 37 & Macao & 1 & 1 \\
\hline Nigeria & 293 & 596 & Papua New Guinea & 22 & 42 & Mongolia & 1 & 1 \\
\hline Malaysia & 260 & 252 & Sierra Leone & 22 & 35 & Niger & 1 & 1 \\
\hline Romania & 258 & 236 & Cameroon & 20 & 21 & Solomon Islands & 1 & 2 \\
\hline Saudi Arabia & 249 & 295 & Ethiopia & 20 & 34 & Somalia & 1 & 4 \\
\hline Morocco & 248 & 254 & Liechtenstein & 20 & 13 & St Vincent \& the Grenadines & 1 & 1 \\
\hline Taiwan & 247 & 270 & Sudan & 20 & 28 & & & \\
\hline Tunisia & 233 & 178 & Aruba & 19 & 6 & & & \\
\hline Angola & 230 & 265 & Yemen & 19 & 19 & & & \\
\hline Croatia & 220 & 195 & Fiji & 18 & 10 & & & \\
\hline Philippines & 217 & 181 & Botswana & 16 & 17 & & & \\
\hline Ukraine & 217 & 152 & Gambia & 15 & 33 & & & \\
\hline Mauritius & 213 & 165 & Turks \& Caicos Islands & 15 & 10 & & & \\
\hline Jamaica & 193 & 395 & Togo & 14 & 5 & & & \\
\hline Kuwait & 193 & 135 & Congo & 13 & 16 & & & \\
\hline Iraq & 192 & 235 & Georgia & 12 & 9 & & & \\
\hline Cuba & 172 & 157 & Panama & 12 & 16 & & & \\
\hline Kenya & 171 & 190 & Uzbekistan & 12 & 5 & & & \\
\hline Gibraltar & 144 & 89 & Macedonia & 11 & 4 & & & \\
\hline Kazakhstan & 139 & 236 & Montenegro & 11 & 14 & & & \\
\hline Sri Lanka & 133 & 103 & Malawi & 10 & 16 & & & \\
\hline
\end{tabular}

1 Any country not included in this table is estimated having

Trade in Services of less than $£ 500,000$

2 United Arab Emirates includes Abu Dhabi, Dubai, Sharjah, Ajman,

Umm al Qaiwain, Ras al Khaimah and Fujairah 


\begin{tabular}{|c|c|c|c|c|c|c|c|c|c|c|c|c|}
\hline & \multicolumn{10}{|c|}{ US\$ million } & \multicolumn{2}{|c|}{$\%$} \\
\hline & \multirow[b]{2}{*}{ World } & \multirow[b]{2}{*}{ US } & \multirow[b]{2}{*}{ Canada } & \multirow[b]{2}{*}{ Japan } & \multirow[b]{2}{*}{ France } & \multirow[b]{2}{*}{ Germany } & \multirow[b]{2}{*}{ Italy } & \multirow[b]{2}{*}{ Russia } & \multirow[b]{2}{*}{$\mathrm{UK}^{2}$} & \multirow[b]{2}{*}{ G8 total } & \multicolumn{2}{|c|}{ The UK as a percentage of: } \\
\hline & & & & & & & & & & & World & G8 \\
\hline \multicolumn{13}{|c|}{ Exports } \\
\hline 2000 & 1508491 & 283778 & 40230 & 69238 & 82703 & 83150 & 56556 & 9565 & 124159 & 749379 & 8.2 & 16.6 \\
\hline 2001 & 1513727 & 271488 & 38804 & 64516 & 82227 & 88714 & 57676 & 11441 & 126393 & 741259 & 8.3 & 17.1 \\
\hline 2002 & 1632231 & 277692 & 40481 & 65712 & 88734 & 103144 & 60439 & 13611 & 141261 & 791074 & 8.7 & 17.9 \\
\hline 2003 & 1881426 & 288348 & 44242 & 77621 & 101585 & 123873 & 71767 & 16229 & 166674 & 890339 & 8.9 & 18.7 \\
\hline 2004 & 2274137 & 335211 & 50286 & 97611 & 114629 & 147498 & 84524 & 20595 & 206697 & 1057051 & 9.1 & 19.6 \\
\hline 2005 & 2538158 & 368343 & 55829 & 110210 & 122221 & 166593 & 89216 & 24970 & 217580 & 1154962 & 8.6 & 18.8 \\
\hline 2006 & 2874551 & 413168 & 60353 & 117298 & 128943 & 194788 & 98984 & 31102 & 249763 & 1294399 & 8.7 & 19.3 \\
\hline 2007 & 3453213 & 484210 & 65338 & 129117 & 149595 & 229156 & 112213 & 39257 & 307647 & 1516533 & 8.9 & 20.3 \\
\hline 2008 & 3883444 & 530102 & 68011 & 148755 & 167639 & 261374 & 120183 & 51132 & 316619 & 1663815 & 8.2 & 19.0 \\
\hline 2009 & 3423629 & 497869 & 59077 & 128338 & 143474 & 231262 & 102637 & 41524 & 262095 & 1466276 & 7.7 & 17.9 \\
\hline \multicolumn{13}{|c|}{ Imports } \\
\hline 2000 & 1527751 & 218252 & 44118 & 116864 & 65509 & 137256 & 55601 & 16230 & 101396 & 755226 & 6.6 & 13.4 \\
\hline 2001 & 1544343 & 216264 & 43843 & 108249 & 67053 & 141916 & 57753 & 20572 & 101614 & 757264 & 6.6 & 13.4 \\
\hline 2002 & 1642178 & 225644 & 45069 & 107940 & 73211 & 145154 & 63166 & 23497 & 111760 & 795441 & 6.8 & 14.1 \\
\hline 2003 & 1873877 & 243478 & 52454 & 111528 & 87565 & 173059 & 74332 & 27122 & 130359 & 899897 & 7.0 & 14.5 \\
\hline 2004 & 2222657 & 281592 & 58776 & 135514 & 99687 & 196756 & 83246 & 33287 & 154859 & 1043717 & 7.0 & 14.8 \\
\hline 2005 & 2464172 & 301649 & 65749 & 134256 & 106962 & 211563 & 90081 & 38745 & 170122 & 1119127 & 6.9 & 15.2 \\
\hline 2006 & 2749115 & 335749 & 72760 & 135556 & 113410 & 226253 & 100511 & 44716 & 183717 & 1212672 & 6.7 & 15.1 \\
\hline 2007 & 3248241 & 366164 & 82788 & 150367 & 129872 & 261298 & 121874 & 58145 & 211648 & 1382156 & 6.5 & 15.3 \\
\hline 2008 & 3706398 & 397226 & 89139 & 169544 & 142013 & 291342 & 130539 & 75468 & 214610 & 1509881 & 5.8 & 14.2 \\
\hline 2009 & 3305784 & 369200 & 78727 & 148718 & 127416 & 254881 & 116663 & 61287 & 172548 & 1329440 & 5.2 & 13.0 \\
\hline
\end{tabular}

Balances

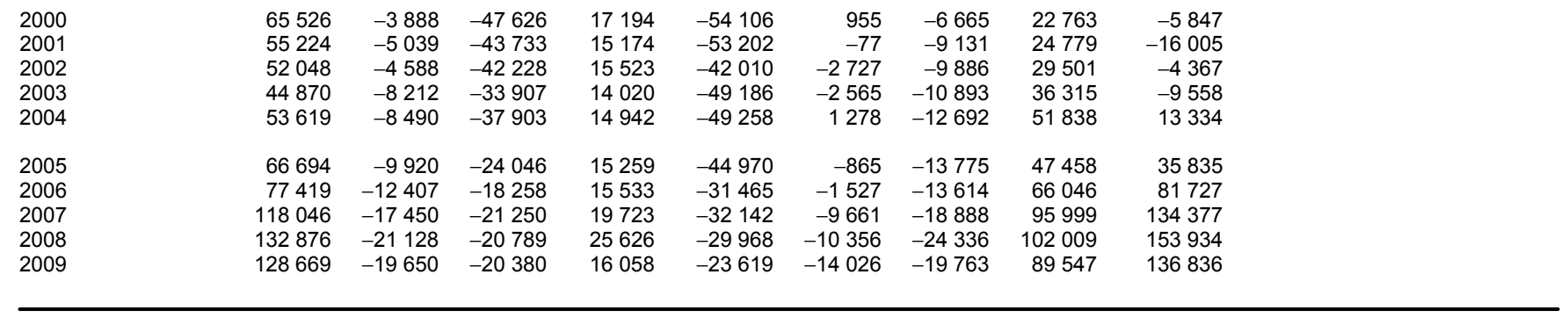

1 G8 country data is not yet available for 2010

Sources: G8 and world data provided by IMF; 